\title{
SOLENOIDAL ATTRACTORS WITH BOUNDED COMBINATORICS ARE SHY
}

\author{
DANIEL SMANIA \\ Dedicated to the memory of \\ Welington de Melo (1946-2016)
}

\begin{abstract}
We show that in a generic finite-dimensional real-analytic family of real-analytic multimodal maps, the subset of parameters on which the corresponding map has a solenoidal attractor with bounded combinatorics is a set with zero Lebesgue measure.
\end{abstract}

\section{Contents}

1. Introduction.

2. Renormalization of extended maps.

3. Complexification of the renormalization operator $\mathcal{R}$. 12

4. Action of $D \mathcal{R}$ on horizontal directions. 15

5. Hyperbolicity of the $\omega$-limit set $\Omega_{n, p}$ of $\mathcal{R}$. 29

6. Induced expanding maps. $\quad 37$

7. Induced problem. $\quad 41$

8. Solving the induced problem. 43

9. Transversal families have hyperbolic parameters. 53

10. Families of multimodal maps $\quad 59$

$\begin{array}{ll}\text { References } & 65\end{array}$

\section{INTRODUCTION.}

A multimodal map $f: I \rightarrow I$ is a smooth map defined in an interval $I$, with a finite number of critical points $c_{i}$, all of them local maximum or local minimum, and such that $f(\partial I) \subset \partial I$. We are going to assume that $f$ is real-analytic.

For unimodal maps with a quadratic critical point, the understanding of the typical behaviour is very satisfactory. Lyubich [26] and Graczyk and Światek [18] proved the density of hyperbolic parameters in the quadratic family. But this was not enough to understand the typical behaviour at almost every parameter of the quadratic family. Indeed earlier Jakobson [20] proved that in the complement of

Date: July 11, 2019.

2000 Mathematics Subject Classification. 37E05, 37E20, 37F25, 37C20, 37D20, 37E20, 37L45.

Key words and phrases. rigidity, renormalization, conjugacy, universality, hyperbolic, solenoidal attractor, multimodal.

We thank the referees for their careful reading and suggestions. D.S. was partially supported by CNPq 430351/2018-6, 307617/2016-5, 470957/2006-9, 310964/2006-7, 472316/03-6, 303669/20098, 305537/2012-1 and FAPESP 03/03107-9, 2008/02841-4, 2010/08654-1, 2017/06463-3. 
the hyperbolic parameters there is a subset of parameters with positive measure for which the dynamics admits an absolutely continuous invariant probability (the map is stochastic). Finally Lyubich [29] proved that for almost every parameter in the quadratic family the map is either regular (a hyperbolic map) or stochastic. Avila, Lyubich and de Melo [3] generalised this result for a non degenerate real analytic family of quadratic real analytic unimodal maps and Avila and Moreira [5] improved this, proving that in a non degenerate family the map is either regular or Collet-Eckmann at almost every parameter. There are similar results for realanalytic unimodal maps with higher order by Clark [11]. See also Bruin, Shen and van Strien [10], Avila, Lyubich and Shen [4] and Shen [38] for related results.

Similar studies for multimodal maps (or even unimodal maps with higher order) pose new difficulties. New phenomena appear, as non-renormalizable maps without decay of geometry (see Bruin, Keller, Nowicki and van Strien [8], Keller and Nowicki [22] ). Decay of geometry was an essential tool in the study of unimodal quadratic maps. This was a major difficulty in the study of the so-called Fibonacci renormalization for unimodal maps with higher order in Smania [42] and the proof of the density of hyperbolicity for polynomials in Kozlovski, Shen, van Strien [24] [23]. Moreover the lack of decay of geometry allows additional metric behaviours, as the existence of wild attractors. See Milnor [33], Bruin, Keller, Nowicki and van Strien [8] and Bruin, Keller and St. Pierre [9].

Another issue is that for families of polynomials with more than one critical point (as in the cubic family) the parameter space has dimension larger than one. That implies that the parapuzzle approach as used in the unimodal case (see Lyubich [28], Avila, Lyubich and de Melo [3]) does not seem to be easily adaptable here, since the fact that holomorphic maps with one-variable are conformal was used in a crucial way.

So as a consequence there are a lot of unanswered questions concerning the typical behaviour in the measure-theoretical sense in families of polynomials and/or multimodal maps.

One of them is how often maps with solenoidal attractors appear in these families. We say that a set $\Lambda \subset I$ is a solenoidal attractor of a multimodal map $f$ if there exists an increasing sequence of positive intergers $n_{k}, k \in \mathbb{N}$, and a family of closed intervals $I_{j}^{k} \subset I, k \in \mathbb{N}$ and $0 \leq j<n_{k}$, such that

A. For each $k$ the intervals in the family $\left\{I_{j}^{k}\right\}_{j<n_{k}}$ has pairwise disjoint interior.

B. We have $f\left(I_{j}^{k}\right) \subset I_{j+1}^{k} \bmod n_{k}$.

C. For every $k$

$$
\left\{c_{i}\right\}_{i} \cap \cup_{j<n_{k}} I_{j}^{k} \neq \emptyset .
$$

and

D. We have

$$
\cup_{j<n_{k+1}} I_{j}^{k+1} \subset \cup_{j<n_{k}} I_{j}^{k}
$$

$$
\Lambda=\cap_{k} \cup_{j<n_{k}} I_{j}^{k} .
$$

See Blokh and Lyubich [6] [7] for more information on attractors for multimodal maps. The solenoidal attractor $\Lambda$ has bounded combinatorics if

$$
\sup _{k} \frac{n_{k+1}}{n_{k}}<\infty \text {. }
$$

One important step in previous results about the typical behaviour in families of unimodal maps is to prove that at a typical parameter the map does not have 
solenoidal attractors. This was done in the quadratic family by Lyubich [28] and for no degenerate families of unimodal maps by Avila, Lyubich and de Melo [3]. An important tool in many of these results on unimodal maps is the fact that the topological classes of unimodal maps extend to an analytic, codimension one lamination (except a few combinatorial types). This implies that the holonomy of this lamination is quite regular. Our goal is to prove that

Theorem A. On a generic real-analytic finite-dimensional family of real-analytic multimodal maps with quadratic critical points and negative schwarzian derivative the set of parameters whose corresponding maps have a solenoidal attractor with bounded combinatorics has zero Lebesgue measure.

The precise statement is given in Theorem 7 . We also have an analogous result for families with finite smoothness and continuous families. The method used in the unimodal case in Avila, Lyubich and de Melo [3] no longer works in the multimodal case, once the lamination of topological classes has higher codimension, so we are going to use a quite different approach. If a map $f$ has a solenoidal attractor with bounded combinatorics, one can find an induced map $F$ of $f$ that is a composition of unimodal maps and it is infinitely renormalizable as defined in [40]. In particular the iterations of the renormalization operator $\mathcal{R}$ for multimodal maps are welldefined for $F$. Using the universality property proved in [40] one can prove that $F$ belongs to the stable lamination of the omega-limit set $\Omega$ of $\mathcal{R}$. The renormalization operator is a real-analytic, compact and non-linear operator acting on a Banach space of real analytic multimodal maps.

Our main technical result is that

Theorem B. Consider the renormalization operator $\mathcal{R}$ acting on real-analytic multimodal maps which are renormalizable with combinatorics bounded by some $p>0$. Then the omega-limit set $\Omega$ of $\mathcal{R}$ is a hyperbolic set.

The precise statement is given in Section 5. Lyubich[27] proved the hyperbolicity of the omega-limit set in the unimodal case using the so-called Small Orbits Theorem. We use a different approach, reducing the study of the hyperbolicity of $\Omega$ to the study of the existence and regularity of solutions for a certain linear cohomological equation. This new method allows us to deal only with real-analytic maps and its complex analytic extensions.

The relationship between renormalization and cohomological equations appears in many contexts, as for instance in the study of rigidity of circle diffeomorphisms and generalized interval exchange transformations. Closer to our setting we have the introduction by Lyubich [27] of the concept of horizontal direction in the study of the renormalization operator for unimodal maps and the study of the hyperbolicity of the fixed point of the action of a pseudo-Anosov map on certain character variety by Kapovich [21].

The final ingredient is a very recent result on partially hyperbolic invariant sets on Banach spaces [43]. The result we use is, roughly speaking, the following (see [43, Theorem 1]). Suppose that a "regular" real-analytic operator $\mathcal{R}$ has a hyperbolic set $\Omega$, and its stable lamination $W^{s}(\Omega)$ satisfies the "Transversal Empty Interior property": every regular manifold $M$ that is transversal to $W^{s}(\Omega)$ intersects $W^{s}(\Omega)$ 
in a subset of empty interior (in the topology of $M$ ). Then a generic real-analytic finite-dimensional family intersects $W^{s}(\Omega)$ on a subset with zero Lebesgue measure. This will give us our main result. The Transversal Empty Interior property for the renormalization operator (see Corollary 9.1) is closely related with the fact that maps $F$ that are infinitely renormalizable with bounded combinatorics can be approximated by hyperbolic maps.

Some of the most classical families of one-dimensional dynamical systems are families of polynomials. The cubic family is the two parameter family

$$
f_{a, b}(z)=z^{3}-3 a^{2} z+b
$$

The critical points of $f_{a, b}$ are $a,-a$. We also have

Theorem C. The set of of parameters $(a, b) \in \mathbb{R}^{2}$ such that $f_{a, b}$ is infinitely renormalizable with bounded combinatorics has zero 2-dimensional Lebesgue measure.

The study of the renormalization operator has a long history. It was first discovered in the unimodal case by Feigenbaum [15][16] and Coullet and Tresser [45]. They conjectured that the period-doubling renormalization operator has a unique fixed point in a space of quadratic unimodal maps, that this fixed point is hyperbolic and its codimension one stable manifold contains all Feigenbaum maps. Such conjectures could explain certain intriguing universal features of the bifurcation diagram of families of unimodal maps. The existence and hyperbolicity of such fixed point was proven by Lanford [25]. Such conjectures were later extended for arbitrary bounded combinatorial types, when the fixed point need to be replaced by an omega-limit set that is hyperbolic (see Derrida, Gervois and Pomeau [14] and Gol'berg, Sinal̆ and Khanin [17]). Sullivan [44] proved that the orbit by the renormalization operator of a map that is infinitely renormalizable with bounded combinatorics converges to the orbit of a map on the omega limit set and such orbit is determined by the combinatorics of the map. This Sullivan's result in particular implies that uniqueness of the fixed point to the period-doubling renormalization operator and that it attracts all Feigenbaum maps. McMullen[32] proved that the rate of convergence is indeed exponential. Finally Lyubich [27] proved that the omega-limit set of the renormalization operator for unimodal maps is hyperbolic. In particular Lyubich found a suitable space where the renormalization operator is a complex-analytic non-linear operator. See also de Faria, de Melo and Pinto [12] for the proof of the conjectures in the $C^{r}$ case.

The renormalization operator for bimodal maps was first considered in MacKay and van Zeijts [30] and $\mathrm{Hu}$ [19]. The general multimodal case, with a precise combinatorial description, was described in [39], as well the so-called real and complex a priori bounds for bounded combinatorics. In [41] it was proved the phase space universality in the bounded combinatorics case.

It is natural to ask if results as Theorems A., B. and C. holds for the full renormalization operator, that is, considering unbounded combinatorial type as well. We believe that recent results by Avila and Lyubich [2] on the contraction of the renormalization operator in the hybrid class of infinitely renormalizable unimodal maps with unbounded combinatorics can be carried out for multimodal maps. So the main difficulty seems to be to understand the dynamics of the renormalization operator in the directions transversal to the horizontal spaces, as in the proof of 


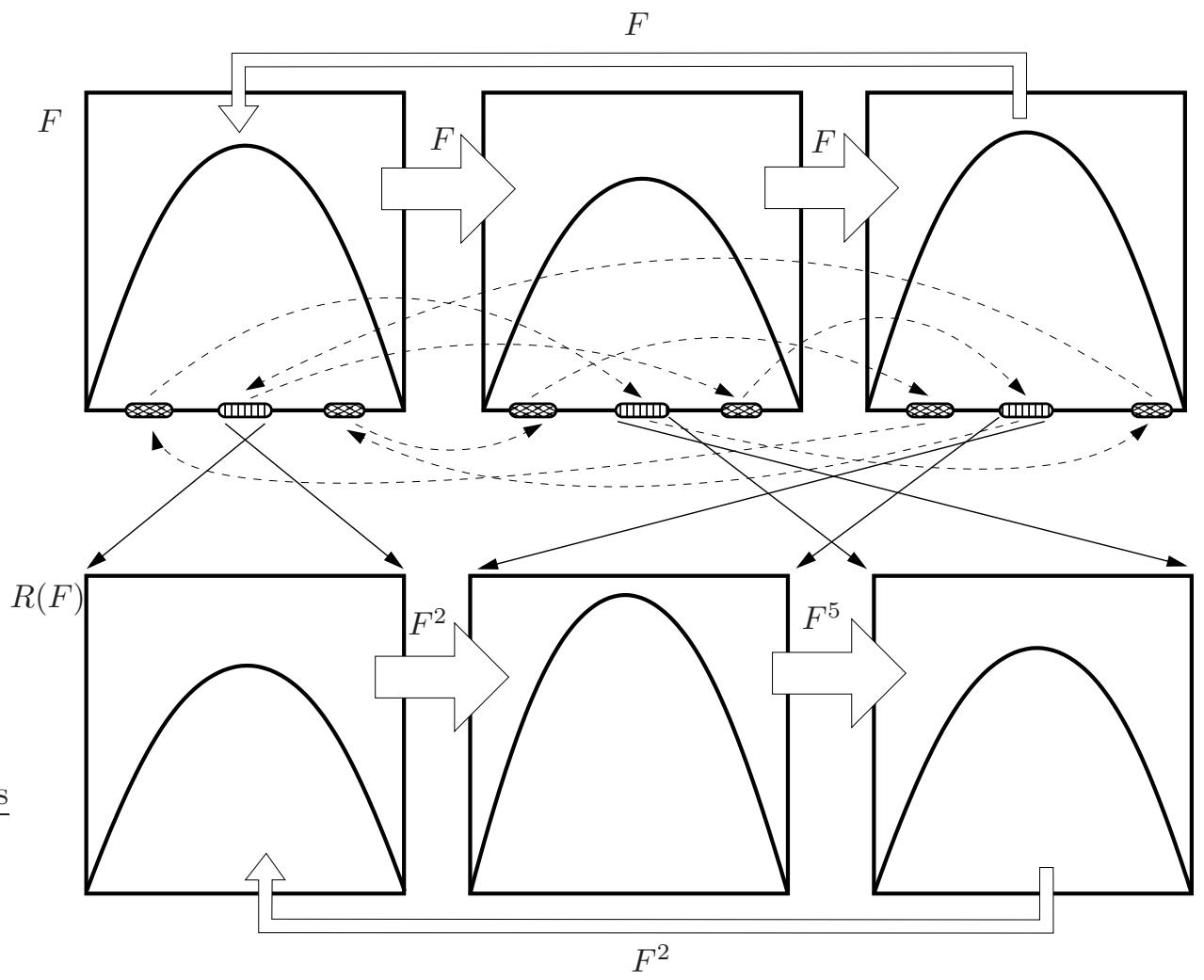

FiguRE 1. Renormalization of an extended map of type 3.

Theorem B. New difficulties arise in the unbounded case, once the omega-limit set of the renormalization operator has not a simple structure anymore. However we are confident that a version of the Key Lemma (Theorem 4) can be obtained in this setting and it will be useful to understand the dynamics of the renormalization operator and the generic behavior in families of multimodal maps.

\section{Renormalization of EXtended maps.}

To study the renormalisation of multimodal maps, it is more convenient to decompose the dynamics of $f$ in its unimodal parts. Let $I_{i}=\left[-1, a_{i}\right]$, with $a_{i}>0$, be intervals and

$$
f_{i}: I_{i} \rightarrow I_{i+1} \bmod n
$$

be $C^{1}$ maps such that $c_{i}$ is its unique critical point, that is a maximum and $f_{i}\left(\partial I_{i}\right) \subset$ $\partial I_{i+1} \bmod n$. An extended map $F$ is defined by a finite sequence $\left(f_{1}, \ldots, f_{n}\right)$ of maps is the map defined on $I_{F}^{n}=\left\{(x, i): x \in I_{i}, 1 \leq i \leq n\right\}$ as

$$
F(x, i)=\left(f_{i}(x), i+1 \bmod n\right)
$$

We say that $f$ is a multimodal map of type $n$ if it can be written as a composition of $n$ unimodal maps: to be more precise, if there exist maps $f_{1}, \ldots, f_{n}$ as above satisfying 
(1) $f=f_{n} \circ \cdots \circ f_{1}$.

(2) We have $f_{i}\left(c_{i}\right) \geq c_{i+1} \bmod n$.

The $n$-uple $\left(f_{1}, \ldots, f_{n}\right)$ is a decomposition of $f$. In this paper, we will assume that the unimodal maps are analytic and the critical points of $f_{i}$ are quadratic. Clearly $f$ has many decompositions.

In [39], we proved that deep renormalizations of infinitely renormalizable multimodal maps are multimodal maps of type $n$.

2.1. Renormalization of extended maps. We say that $J$ is a $k$-periodic interval, $k \geq 2$, of the extended map $F$ if

- $\left(c_{1}, 1\right) \in J\left(\left(c_{i}, i\right)\right.$ are the critical points of $\left.F\right)$,

- $\left\{J, F(J), \ldots, F^{k-1}(J)\right\}$ is a collection of intervals with disjoint interiors,

- The union of intervals in the above family contains $\left\{\left(c_{i}, i\right)\right\}$,

- $F^{k}(J) \subset J$.

We will call $k$ the period of $J$. If $F$ has a $k$-periodic interval, for some $k$, we say that $F$ is renormalizable.

Suppose that there exists a $k$-periodic interval for $F$. Let $P \subset I_{1} \times\{1\}$ be the maximal interval which is a $k$-periodic interval for $F$. Then $F^{k}(\partial P) \subset \partial P$. We say that $P$ is a restrictive interval for $F$ of period $k$. Note that if $P$ and $\tilde{P}$ are, respectively, restrictive intervals for $F$ of period $k$ and $\tilde{k}, k<\tilde{k}$, then $\tilde{P} \subset P$. Let $P$ be a restrictive interval and let $0=\ell_{1}<\cdots<\ell_{n}$ be the iterations such that $\left(c_{i}, i\right) \in F^{\ell_{j}}(P)$ for some $i$. Let $P_{j}$ be the symmetrization of $F^{\ell_{j}}(P)$ in relation to $\left(c_{i}, i\right)$. Observe that $P_{j}$ contains a periodic point in its boundary. If $\left(c_{i}, i\right) \in P_{j}$ Let

$$
A_{P_{j}}: \mathbb{C} \times\{i\} \rightarrow \mathbb{C} \times\{j\}
$$

be the affine map which maps $\left(c_{i}, i\right)$ to $(0, j)$ and this periodic point to -1 . Let $\left[-1, b_{j}\right] \times\{j\}=A_{P_{j}}\left(P_{j}\right)$. Then

$$
g_{j}:\left[-1, b_{j}\right] \times\{j\} \rightarrow\left[-1, b_{j+1}\right] \times\{j+1\}
$$

defined by $g_{j}=A_{P_{j+1}} \circ F^{\ell_{j+1}-\ell_{j}} \circ A_{P_{j}}^{-1}$ is a unimodal map. The extended map $G(x, j)=g_{j}(x, j)$ is called a renormalization of the extended map $F$. An extended map may have many renormalizations, but at most one with a given period. The renormalization with minimal period $k$ is called the first renormalization of $F$, and it is denoted $R(F)$.

Following the notation in [40], the primitive marked combinational data (primitive m.c.d) associated with the first renormalization of $F$ is $\sigma=\left\langle A, \prec, A^{c}>\right.$ where

- $A=\{1,2, \ldots, k\}$,

- The relation $\prec$ is a partial order on $A$ defined in the following way $i \prec \ell$ if $F^{i} J$ and $F^{\ell} J$ belongs to the same interval in $I_{F}^{n}$ and $F^{i} J$ is on the left side of $F^{\ell} J$,

- The set $A^{c}$ is a subset of $A$ and $i \in A^{c}$ if $F^{i} J$ intersects $\left\{\left(c_{i}, i\right)\right\}$.

The extended map $R(F)$ can be renormalizable again and so on. If this process can be continued indefinitely, we say that $F$ is infinitely renormalizable. If $F$ is infinitely renormalizable then all of its renormalizations can be obtained iterating the operator $R$. Denote by $P_{0}^{k}$ the restrictive interval associated to the $k$-th renormalization $R^{k}(F)$. If $q \in C(F):=\left\{\left(c_{i}, i\right)\right\}$, denote by the corresponding capital letter $Q_{0}^{k}$ the symmetrization of the interval $F^{\ell}\left(P_{0}^{k}\right)$ which contains $q$. We reserve 
the letter $p$ for $\left(c_{1}, 1\right)$. The critical point $r$ for $F$ will be the successor of the critical point $q$ at level $k$ if $r \in F^{\ell}\left(Q_{0}^{k}\right)$, for the minimal $\ell$ so that $F^{\ell}\left(Q_{0}^{k}\right)$ contains a critical point. Define $n_{r}^{k}=\ell$. Then, for any $r \in C(F), k \in \mathbb{N}$ and $i<n_{r}^{k}$, there exists an interval $R_{-i}^{k}$ so that

- $F^{i}$ is monotone in $R_{-i}^{k}$,

- $F^{i}\left(R_{-i}^{k}\right)=R_{0}^{k}$,

- The interval $F_{r}^{n_{r}^{k}-i}\left(Q_{0}^{k}\right)$ is contained in $R_{-i}^{k}$.

For details, see [39].

Denote by $N_{k}$ the period of the restrictive interval $P_{0}^{k}$. We say that $F$ has C-bounded combinatorics if $N_{k+1} / N_{k} \leq C$ for every $k$.

For $(x, i),(y, j) \in I_{F}^{n}$, we say that $(x, i)<(y, j)$ if $i=j$ and $x<y$. The intervals of $I_{F}^{n}$ are the sets $J \times\{i\}$, for some interval $J \subset I_{i}$ and $1 \leq i \leq n$. If $c_{i}$ is the critical point of $f_{i}$, denote $C(F)=\left\{\left(i, c_{i}\right)\right\}_{i}$.

Let $F$ and $G$ be two infinitely renormalizable extended maps. We say that $F$ and $G$ have same combinatorics if $F^{i}\left(c_{k}\right)<F^{j}\left(c_{\ell}\right)$ if and only if $G^{i}\left(c_{k}\right)<G^{j}\left(c_{\ell}\right)$, for any $i, j \geq 0$ and $k$ and $\ell<n$.

Let $\sigma_{i}$ be the primitive m.c.d. of the (first) renormalization of $R^{i}(F)$ and $\tilde{\sigma}_{i}$ be the primitive m.c.d. of the (first) renomalization of $R^{i}(G)$. It turns out that $F$ and $G$ has the same combinatorics if and only if $\sigma_{i}=\tilde{\sigma}_{i}$ for every $i$. So we say that $F$ has combinatorics $\left(\sigma_{1}, \sigma_{2}, \sigma_{3}, \ldots\right)$. Moreover, let $\mathcal{C}_{p, n}$ be the set of all primitive m.c.d. that appears as the first renormalization of an extended map with $n$ intervals and it has period either smaller or equal to $p$. By Corollary 2.3 in [40] for every given sequence $\sigma_{i} \in \cup_{p} \mathcal{C}_{p, n}, i \geq 1$, there exists a real analytic extended map (with, say, quadratic critical points) whose $i$-th renormalization has primitive m.c.d. $\sigma_{i}$.

2.2. Polynomial-like extended maps. Denote $\mathbb{C}_{n}=\{(x, i): x \in \mathbb{C}, 1 \leq i \leq n\}$ (in other words, $\mathbb{C}_{n}$ is a disjoint union of $n$ copies of $\mathbb{C}$ ). Given an open set $O \subset \mathbb{C}_{n}$, denote

$$
O_{i}=O \cap(\mathbb{C} \times\{i\})
$$

A polynomial-like extended map is a map $F: U \rightarrow V$, where

- $U$ and $V$ are open sets of $\mathbb{C}_{n}$, where $\bar{U} \subset V$,

- for each $i, F\left(U_{i}\right)=V_{i+1} \bmod n$. Moreover $F: U_{i} \rightarrow V_{i+1} \bmod n$ is a proper map with a unique critical point.

- for each $i$ we have that $U_{i}$ and $V_{i}$ are simply connected domains.

We define

$$
\bmod (V \backslash U)=\min _{i} \bmod \left(V_{i} \backslash U_{i}\right) .
$$

The filled-in Julia set $K(F)$ of a polynomial-like extended map $F$ is defined as

$$
K(F)=\cap_{i \geq 0} F^{-i}(V) .
$$

Note that $K(F)$ is connected if and only if all the critical points of $F$ belongs to $K(F)$.

A real analytic extended map $F: I_{F}^{n} \rightarrow I_{F}^{n}$ has a polynomial-like extension if there is a polynomial-like extend map $\tilde{F}: U \rightarrow V$ such that $I \times\{i\} \subset U_{i}$ and $F=\tilde{F}$ on $I_{F}^{n}$. 
2.3. Polynomial-like renormalization of real analytic extended maps. Let $U \subset \mathbb{C}_{n}$ be an open set. Given an analytic function $F: U \rightarrow \mathbb{C}_{n}$, define the open set

$$
\mathcal{D}_{U}^{n}(F):=\bigcap_{i=0}^{n-1} F^{-i} U
$$

In other words, $\mathcal{D}_{U}^{n}(F)$ is the domain contained in $\mathbb{C}_{n}$ where $F^{n}$ is defined.

Let $F: U \rightarrow V$ be a complex-analytic extension of an extended map. Note that $F$ does not need to be a polynomial-like extension. Suppose that the extended map $F$ is $r$-times renormalizable, and let $P_{j}$ and $\ell_{j}$ be the intervals and integers associated with the $r$ th renormalization, as defined in Section 2.1. Define $n_{k}=\ell_{k+1} \bmod n-\ell_{k}$.

Suppose we can find a sequence $i_{k}, k=1, \ldots, n$, with $i_{1}=1$, simply connected domains $\hat{U}_{k}$ and $\hat{V}_{k} \subset \mathbb{C} \times\left\{i_{k}\right\}$, such that

1. We have $\left\{i_{k}\right\}_{k}=\{1,2, \ldots, n\}$,

2. We have $P_{k} \subset \hat{U}_{k}$ and $\overline{\hat{U}_{k}} \subset \hat{V}_{k}$,

3. The domains $\hat{U}_{k}$ and $\hat{V}_{k}$ satisfies $\overline{\hat{U}_{k}} \subset \mathcal{D}_{U}^{n_{k}}(F), F^{n_{k}} \hat{U}_{k}=\hat{V}_{k+1} \bmod n$.

4. The map

$$
F^{n_{k}}: \hat{U}_{k} \rightarrow \hat{V}_{k+1} \bmod n
$$

is a proper map for which $\left(0, i_{k}\right)$ is the unique critical point.

Let $A_{j}: \mathbb{C} \rightarrow \mathbb{C}$ be the affine maps defined in Section 2.1. Define $A: \mathbb{C}_{n} \rightarrow \mathbb{C}_{n}$ as $A(x, i)=\left(A_{i}(x), i\right)$. Then we can define

$$
g_{i}: A\left(\hat{U}_{j}\right) \rightarrow A\left(\hat{V}_{j+1}\right)
$$

as $g_{j}=A \circ F^{n_{k}} \circ A^{-1}$. If

$$
\hat{U}=\bigcup_{i} A\left(\hat{U}_{i}\right) \times\{i\}, \hat{V}=\bigcup_{i} A\left(\hat{V}_{i}\right) \times\{i\},
$$

then the map

$$
R^{r}(F): \hat{U} \rightarrow \hat{V}
$$

defined by $R^{r}(F)(x, i)=\left(g_{i}(x), i+1\right)$ is a polynomial-like extension of the real renormalization $R^{r}(F)$ and it is called a polynomial-like $r$ th renormalization of $F$. Note that $\hat{U}_{k}$ and $\hat{V}_{k}$ are not uniquely defined, however any polynomial-like extension of $R^{r}(F)$ does coincide on $I_{R^{r}(F)}^{n}$.

Lemma 2.1. Let $x_{1}, \ldots, x_{n} \in \mathbb{C}$, and $U_{i} \subset \mathbb{C}$ be open sets such that $x_{i} \in U_{i_{k}}$ and

$$
f_{i, k}: U_{i, k} \rightarrow \mathbb{C}, k=1,2,
$$

be holomorphic functions such that

i. $f_{i, k}\left(x_{i}\right)=x_{i+1} \bmod n$.

ii. We have $\left|\lambda_{1} \lambda_{2} \cdots \lambda_{n}\right|>1$, where $\lambda_{i}=f_{i, 1}^{\prime}\left(x_{i}\right)=f_{i, 2}^{\prime}\left(x_{i}\right)$.

iii. Let

$$
g_{i, k}=f_{(i+n-1) \bmod n, k} \circ \cdots \circ f_{(i+1) \bmod n, k} \circ f_{i, k} .
$$

There is $q \geq 1$ such that $g_{i, 1}^{q}=g_{i, 2}^{q}$ for every $i$.

Then $f_{i, 1}(x)=f_{i, 2}(x)$ for every $i$ and for every $x$ close to $x_{i}$. 
Proof. Due iii. we have that $x_{i}$ is a repelling fixed point of $g_{i, k}$ and its multiplier is $\lambda_{1} \lambda_{2} \cdots \lambda_{n}$. By the Kœnigs linearization theorem (see for instance Milnor [34, Theorem 8.2]) there is a unique germ of holomorphic function $h_{i}$ at $x_{i}$ such that $h_{i}^{\prime}\left(x_{i}\right)=1$ and

$$
h_{i} \circ g_{i, 1}(x)=g_{i, 2} \circ h_{i}(x)
$$

for $x$ close to $x_{i}$. Note that the uniqueness of $h_{i}$ and ii. implies

$$
h_{i+1} \circ f_{i, 1}(x)=f_{i, 2} \circ h_{i}(x) .
$$

for $x$ close to $x_{i}$. Note also that

$$
h_{i} \circ g_{i, 1}^{q}(x)=g_{i, 2}^{q} \circ h_{i}(x)
$$

But since $g_{i, 1}^{q}=g_{i, 2}^{q}$ this implies (due the uniqueness of the solution of the Schröder's equation in the Konigs linearization theorem) that $h_{i}(x)=x$, so by (4) we have $f_{i, 1}=f_{i, 2}$ for every $i$.

Proposition 2.2 (Injectivity of Renormalization). Let $F_{1}, F_{2}$ be real-analytic extended maps of type $n$ with polynomial-like extensions of type $n$. Suppose that $F_{k}$, $k=1,2$ are renormalizable and $R\left(F_{k}\right), k=1,2$, also have polynomial-like extensions of type $n$. Additionally assume that

$$
r \in \overline{\left\{F_{k}^{\ell}(s), \ell \geq 0\right\}} \text {, every } r, s \in C\left(F_{k}\right), k=1,2,
$$

Then $R\left(F_{1}\right)=R\left(F_{2}\right)$ implies $F_{1}=F_{2}$.

Proof. We use an argument similar to de Melo and van Strien [13, Chapter VI, Proposition 1.1]. To simplify the notation we assume that $F_{k}(x, i)=F_{k}(-x, i)$. Let $p_{k}$ be the period of the first renormalization of $F_{k}$ and $q_{k}=p_{k} / n$. Using the notation of Section 2.1, we have $c_{i}=0, a_{i}=1$ and $b_{i}=1$, for every $i \leq n$. Let $P_{1, k}$ be the interval of the first renormalization of $F_{k}$ such that that $(0,1) \in P_{1, k}$. Let $0=\ell_{1, k}<\cdots<\ell_{n, k}$ be the iterations such that $\left(0, i_{j, k}\right) \in F_{k}^{\ell_{j, k}}\left(P_{1, k}\right)$ for some $i_{j, k}$. Let $P_{j, k}=\left[-\beta_{j, k}, \beta_{j, k}\right]$ be the symmetrization of $F_{k}^{\ell_{j, k}}\left(P_{j, k}\right)$, where $\beta_{j, k}$ is periodic. Let

The map

$$
Y_{j, k}=\left[-\frac{1}{\beta_{j, k}}, \frac{1}{\beta_{j, k}}\right] .
$$

defined by

$$
g_{j, k}: Y_{j, k} \rightarrow Y_{j, k}
$$

$$
g_{j, k}(x)=\frac{-1}{\beta_{j, k}} \pi_{1}\left(F_{k}^{n}\left(-\beta_{j, k} x, i_{j_{k}}\right)\right) .
$$

is a multimodal map with a polynomial-like extension of degree $2^{n}$ and the real trace of its filled-in Julia set is $\left[-1 / \beta_{j, k}, 1 / \beta_{j, k}\right]$. Then $R\left(F_{1}\right)=R\left(F_{2}\right)$ implies that $g_{j, 1}^{q_{1}}=g_{j, 2}^{q_{2}}$ on $[-1,1]$ and for every $j$. Moreover $g_{j, k}^{q_{k}}$ has a polynomial-like extension of degree $2^{n}$ whose real trace of its filled-in Julia set is $[-1,1]$. Note that if $\left|\beta_{j, 1}\right|<\left|\beta_{j, 2}\right|$ then $Y_{j, 2}$ is invariant by $g_{j, 1}^{q_{1}}$, that implies that $Y_{j, 2}$ would be a restricted interval of $g_{j, 1}$, which is not possible since $g_{j, 1}^{q_{1}}$ on $[-1,1]$ is the first renormalization of $g_{j, 1}$. So

$$
\beta_{j, 1}=\beta_{j, 2} \text { and } Y_{j, 1}=Y_{j, 2} \text { for every } j
$$

Counting the number of restricted intervals associated to $\left[-\beta_{j, k}, \beta_{j, k}\right]$ in $Y_{2, k}$ we obtain $p_{1}=p_{2}$. 
Note that $\ell_{1, k}$ is the number of critical values of $F_{k}^{\ell_{j, k}}$ in $[-1,1] \times\{1\}$, which is equal to the number of critical values of $R\left(F_{k}\right)$ in $Y_{1, k} \times\{1\}$. Since $R\left(F_{1}\right)=R\left(F_{2}\right)$ and $Y_{1,2}=Y_{1,2}$ we conclude that $\ell_{1,1}=\ell_{1,2}$ and $i_{2, k}=1+\ell_{1,1}$ for $k=1,2$. Suppose by induction that $i_{j, 1}=i_{j, 2}$. Then $\ell_{j, k}$ is the number of critical values of $R\left(F_{k}\right)$ in $Y_{j, k} \times\{j\}$, so $\ell_{j, 1}=\ell_{j, 2}$ and consequently $i_{j+1, k}=i_{j+1,1}+\ell_{j, 1}$. So

$$
i_{j, 1}=i_{j, 2} \text { and } \ell_{j, 1}=\ell_{j, 2} \text { for every } j .
$$

Finally due (7), (8) and $R\left(F_{1}\right)=R\left(F_{2}\right)$ we have that

$$
F_{1}^{\ell_{j+1,1}-\ell_{j, 1}}=F_{2}^{\ell_{j+1,2}-\ell_{j, 2}}
$$

in a neighborhood of the point $\left(-1, i_{j, 1}\right)$ and $F_{1}^{\ell_{j+1,1}-\ell_{j, 1}}\left(-1, i_{j, 1}\right)=\left(-1, i_{j+1,1}\right)$ for every $j$.

Let $\lambda_{i, k}=D F_{k}(-1, i)>0$. We claim that $\lambda_{i, 1}=\lambda_{i, 2}$ for every $i$. Indeed, let $p=p_{1}=p_{2}, q=q_{1}=q_{2}$ and $\ell_{i}=\ell_{i, 1}=\ell_{i, 2}$. So $g_{j, 1}^{q}=g_{j, 2}^{q}$ and (7) implies that $F_{1}^{q n}=F_{2}^{q n}$ in a neighborhood of $[-1,1] \times\{1, \ldots, n\}$. In particular

$$
\left(\lambda_{1,1} \cdots \lambda_{n, 1}\right)^{q}=\left(\lambda_{1,2} \cdots \lambda_{n, 2}\right)^{q},
$$

so

$$
\lambda_{1,1} \cdots \lambda_{n, 1}=\lambda_{1,2} \cdots \lambda_{n, 2} .
$$

There is exactly one $j \leq n$ such that $(0,1) \in F_{1}^{\ell_{j}}\left(P_{1,1}\right)$, that is the unique $i_{1}$ satisfying $\ell_{i_{1}}=w_{1} n+1$, for some $w_{1} \in \mathbb{N}$. In particular

$$
D F_{k}^{\ell_{i_{1}}}(-1,0)=\left(\lambda_{1, k} \cdots \lambda_{n, k}\right)^{w_{1}} \lambda_{1, k} .
$$

Due (9) we have $D F_{1}^{\ell_{i_{1}}}(-1,0)=D F_{2}^{\ell_{i_{1}}}(-1,0)$, so it follows from (10) that $\lambda_{1,1}=$ $\lambda_{1,2}$. Suppose by induction that $\lambda_{j, 1}=\lambda_{j, 2}$ for $j<j_{0}<n$. Then there is a unique $\ell_{i_{j_{0}}}$ such that $\ell_{i_{j_{0}}}=w_{j_{0}} n+j_{0}$ for some $w_{j_{0}} \in \mathbb{N}$ and consequently

$$
D F_{k}^{\ell_{i_{j}}}(-1,0)=\left(\lambda_{1, k} \cdots \lambda_{n, k}\right)^{w_{j_{0}}} \lambda_{1, k} \lambda_{2, k} \cdots \lambda_{j_{0}-1, k} \lambda_{j_{0}, k} .
$$

It follows from the induction assumption, $D F_{1}^{\ell_{i_{0}}}(-1,0)=D F_{2}^{\ell_{i_{0}}}(-1,0)$ and $(10)$ that $\lambda_{j_{0}, 1}=\lambda_{j_{0}, 2}$. So $\lambda_{j, 1}=\lambda_{j, 2}$ for every $j \leq n-q$. We conclude that $\lambda_{n, 1}=\lambda_{n, 2}$ due (10). This concludes the proof of the claim.

Define $f_{i, k}(x)=\pi_{1}\left(F_{k}(x, i)\right)$ and $x_{i}=-1$. By Lemma 2.1 we have that $f_{i, 1}(x)=$ $f_{i, 2}(x)$ for every $i$ and $x$ close to -1 , so $F_{1}=F_{2}$.

2.4. Complex bounds and rigidity of real analytic, infinitely renormalizable extended maps with bounded combinatorics. Here we summarize results in [40].

Theorem 1. Let $\sigma=\left(\sigma_{i}\right)_{i \in \mathbb{Z}} \in \mathcal{C}_{p, n}^{\mathbb{Z}}$. Then there exists a unique sequence of real analytic maps $F_{\sigma, i}, i \in \mathbb{Z}$, satisfying the following conditions

1. The map $F_{\sigma, i}$ is renormalizable and $R\left(F_{\sigma, i}\right)=F_{\sigma, i+1}$.

2. The first renormalization of $F_{\sigma, i}$ has combinatorics $\sigma_{i}$.

3. There exist polynomial-like extensions $F_{\sigma, i}: U_{\sigma}^{i} \rightarrow V_{\sigma}^{i}$, where

$$
\inf _{i} \bmod \left(V_{\sigma}^{i} \backslash U_{\sigma}^{i}\right)>0
$$


If $U \subset \mathbb{C}$ is a bounded open set such that $0 \in U$, denote by $\mathcal{B}(U)$ the Banach space of all holomorphic functions $g: U \rightarrow \mathbb{C}$ that has a continuous extension to $\bar{U}$ and a critical point at 0 , with the sup norm. If $-1 \in \bar{U}$ let $\mathcal{B}_{\text {nor }}(U)$ be the affine subspace of maps $g \in \mathcal{B}(U)$ such that $g(-1)=-1$.

In an analogous way, let $U \subset \mathbb{C}_{n}$ be a bounded open set such that

$$
U_{i}=(\mathbb{C} \times\{i\}) \cap U \neq \emptyset
$$

and $(0, i) \in U$, for every $i$ and consider the set $\mathcal{B}(U)$ of all holomophic functions $G: U \rightarrow \mathbb{C}_{n}$ with the following properties:

1. $G$ has a continuous extension to $\bar{U}$.

2. $G$ has critical points at $(0, i)$, for every $i$.

3. $G\left(U_{i}\right) \subset \mathbb{C} \times\{i+1 \bmod n\}$.

Theorem 2. (Complex Bounds [39][40]) There exists $\epsilon_{0}>0$ with the following property. If $F$ is a real analytic extended map that is infinitely renormalizable with combinatorics in $\mathcal{C}_{p, n}^{\mathbb{N}}$ and with a complex analytic (but not necessarily polynomiallike) extension $F \in \mathcal{B}(U)$ then there exist a neighbourhood $V_{F} \subset \mathcal{B}(U)$ of $F$ and $k_{0}$ with the following property. For every $k \geq k_{0}$ and every real analytic and infinitely renormalizable $G \in V_{F}$ with combinatorics in $\mathcal{C}_{p, n}^{\mathbb{N}}$ the map $G$ has a polynomial-like $k$ th renormalization

$$
R^{k}(G): U^{k} \rightarrow V^{k}
$$

such that

$$
\bmod \left(V^{k} \backslash U^{k}\right)>\epsilon_{0} .
$$

If $(-1, i) \in \bar{U}$ for every $i$, we can also consider the subset $\mathcal{B}_{\text {nor }}(U)$ of all maps $G \in \mathcal{B}(U)$ such that $G(-1, i)=(-1, i+1 \bmod n)$ for every $i$.

Denote $\pi(x, i)=x$. We identify $\mathcal{B}(U)$ with the Banach space

$$
\mathcal{B}\left(\pi\left(U_{1}\right)\right) \times \mathcal{B}\left(\pi\left(U_{2}\right)\right) \times \cdots \times \mathcal{B}\left(\pi\left(U_{n}\right)\right)
$$

in the following way. For each $G \in \mathcal{B}(U)$ there is a unique decomposition

$$
\left(g_{1}, \ldots, g_{n}\right) \in \mathcal{B}\left(\pi\left(U_{1}\right)\right) \times \mathcal{B}\left(\pi\left(U_{2}\right)\right) \times \cdots \times \mathcal{B}\left(\pi\left(U_{n}\right)\right),
$$

where $g_{i}$ is defined by $g_{i}(x)=\pi \circ G(x, i)$ and for each $n$-uple as in (12) we can associate $G \in \mathcal{B}(U)$ defined by $G(x, i)=\left(g_{i}(x), i+1 \bmod n\right)$. With this identification $\mathcal{B}_{\text {nor }}(U)$ turns out to be an affine subspace of $\mathcal{B}(U)$. So given $F \in \mathcal{B}_{\text {nor }}(U)$ we can consider the tangent space of $\mathcal{B}_{\text {nor }}(U)$ at $F$, denoted by $T_{F} \mathcal{B}_{\text {nor }}(U)$. using the identification (11) then $T_{F} \mathcal{B}_{\text {nor }}(U)$ is the subspace of

$$
\left(v_{1}, \ldots, v_{n}\right) \in \mathcal{B}\left(\pi\left(U_{1}\right)\right) \times \mathcal{B}\left(\pi\left(U_{2}\right)\right) \times \cdots \times \mathcal{B}\left(\pi\left(U_{n}\right)\right)
$$

such that $v_{i}(-1)=0$ for $i=1, \ldots, n$. In particular $T_{F} \mathcal{B}_{\text {nor }}(U)$ does not depend on $F$, so sometimes we will write $T \mathcal{B}_{\text {nor }}(U)$.

Given $\delta>0$ and $\theta>0$, let $D_{\delta, \theta}$ be the set

$$
\{x \in \mathbb{C}: \operatorname{dist}(x,[-1,1])<\delta \text { and }|\operatorname{Im}(x)|<\theta(\operatorname{Re}(x)+1)\} \times\{1, \ldots, n\}
$$

Define

Indeed, due Theorem 1 we have that

$$
\Omega_{p, n}=\left\{F_{\sigma, 0}\right\}_{\sigma \in \mathcal{C}_{p, n}^{Z}}
$$

$$
\Omega_{p, n}=\left\{F_{\sigma, i}\right\}_{\sigma \in \mathcal{C}_{p, n}^{\mathbb{Z}}}
$$


for every $i$. Using Theorem 2 and Theorem 1 one can show that there exists $\epsilon_{0}$ such that for every $F_{\sigma, 0} \in \Omega_{p, n}$ there exists a polynomial-like extension $F_{\sigma_{0}}: U_{\sigma}^{0} \rightarrow V_{\sigma}^{0}$ such that $\bmod \left(V_{\sigma}^{0} \backslash U_{\sigma}^{0}\right)>\epsilon_{0}$. There exists $\delta_{0}$ such that for every simply connected domains $Q \supset W \supset[-1,1]$ such that $\bmod (Q \backslash W) \geq \epsilon_{0} / 2$ we have

$$
\left\{x \in \mathbb{C}: \operatorname{dist}(x,[-1,1]) \leq \delta_{0}\right\} \subset Q .
$$

In particular

$$
\overline{D_{\delta_{0}, \theta}} \subset U_{\sigma}^{0}
$$

for every $\theta>0$ and for every $\sigma \in \mathcal{C}_{p, n}$. In particular $\Omega_{p, n} \subset \mathcal{B}_{\text {nor }}\left(D_{\delta_{0}, \theta}\right)$.

Consider the shift operator on $\mathcal{C}_{p, n}^{\mathbb{Z}}$, that is, if $\sigma=\left(\sigma_{i}\right)_{i \in \mathbb{Z}}$ then $S(\sigma)=\sigma^{\prime}$, where $\sigma_{i}^{\prime}=\sigma_{i+1}$.

Corollary 2.3. The set $\Omega_{p, n} \subset \mathcal{B}_{\text {nor }}\left(D_{\delta_{0}, \theta}\right)$ is a Cantor set. Indeed the map $H: \mathcal{C}_{p, n}^{\mathbb{Z}} \rightarrow \Omega_{p, n}$ given by

$$
H(\sigma)=F_{\sigma, 0},
$$

is a homeomorphism. Moreover $R\left(F_{\sigma, 0}\right)=F_{S(\sigma), 0}$.

Proof. The map $H$ is continuous and onto due [40, Section 7.1]. The injectivity of $H$ follows from Proposition 2.2.

\section{Complexification of the Renormalization operator $\mathcal{R}$.}

Given $\theta_{0}>0$, by Theorem 2 for each $F \in \Omega_{p, n}$ there exist a neighbourhood $V_{F} \subset \mathcal{B}_{\text {nor }}\left(D_{\delta_{0}, \theta_{0}}\right)$ of $F$ and $k_{F}$ such that for every real map $G \in V_{F}$ that is infinitely renormalizable with combinatorics in $\mathcal{C}_{p, n}$ and for every $k \geq k_{F}$ we have a polynomial-like $k$ th renormalization $R^{k}(G): \hat{U} \rightarrow \hat{V}$ with $\bmod (\hat{V} \backslash \hat{U})>\epsilon_{0}$. In particular $R^{k}(G) \in \mathcal{B}_{\text {nor }}\left(D_{\delta_{0}, \theta_{0}}\right)$. Since $\Omega_{p, n}$ is a compact set, choose a finite sub cover $\left\{V_{F_{i}}\right\}_{i \leq \ell}$ of $\Omega_{p, n}$. Let $k_{0}=\max _{i \leq \ell} k_{F_{i}}$ and $\mathcal{V}=\cup_{i \leq \ell} V_{F_{i}}$.

Let $H$ be the homeomorphism defined in Corollary 2.3. For every $\gamma=\left(\gamma_{1}, \ldots, \gamma_{k_{0}}\right) \in$ $\mathcal{C}_{p, n}^{k_{0}}$ define the compact set

$$
\Omega_{p, n}(\gamma)=H\left(\left\{\sigma \in \mathcal{C}_{p, n}^{\mathbb{Z}}: \sigma_{i}=\gamma_{i} \text { for } 1 \leq i \leq k_{0}\right\}\right) .
$$

We have

$$
d_{1}=\inf \left\{\operatorname{dist}_{\mathcal{B}_{\text {nor }}\left(D_{\delta_{0}, \theta_{0}}\right)}\left(G_{1}, G_{2}\right): G_{1} \in \Omega_{p, n}(\hat{\gamma}), G_{2} \in \Omega_{p, n}(\tilde{\gamma}), \hat{\gamma} \neq \tilde{\gamma}\right\}>0 .
$$

Given $F \in \Omega_{p, n}$, consider the intervals $P_{F, j}, j=1, \ldots, n$, integers $n_{j}$, correponding the restrictive intervals of the $k_{0}$ th renormalization of $F$, as in Section 2.3. Each interval $P_{F, j}$ contains a unique repelling periodic point $\left(\beta_{F, j}, i_{j}\right)$ in its boundary. These repelling periodic points have a complex analytic continuation $\left(\beta_{G, j}, i_{j}\right)$ for every $G$ in a connected neighbourhood $\tilde{W}_{F}$ of $F$ in $\mathcal{B}_{\text {nor }}\left(D_{\delta_{0}, \theta_{0}}\right)$ that is also a repelling periodic point for $G$. Note that for a real map $G$ the point $\beta_{G, j}$ is real and we can assume that it has the same combinatorics as $\beta_{F, j}$. We can also assume that $\tilde{W}_{F} \subset \mathcal{V}$ and that the diameter of $\tilde{W}_{F}$ is smaller than $d_{1} / 2$.

Let $d_{2}<d_{1}$ be a Lebesgue number of the cover $\left\{\tilde{W}_{F}\right\}_{F \in \Omega_{p, n}}$ of $\Omega_{p, n}$. For every $F \in \Omega_{p, n}$ choose a connected neighbourhood $W_{F} \subset \tilde{W}_{F}$ of $F$ so that

$$
\operatorname{diam}_{\mathcal{B}_{\text {nor }}\left(D_{\delta_{0}, \theta_{0}}\right)} W_{F}<d_{2} / 4
$$

Let $F_{1}, F_{2} \in \Omega_{p, n}$ and consider the complex analytic continuations $\left(\beta_{G, j}^{1}, i_{j}^{1}\right)$, $\left(\beta_{G, j}^{2}, i_{j}^{2}\right)$ of $\left(\beta_{F_{1}, j}, i_{j}^{1}\right)$ and $\left(\beta_{F_{2}, j}, i_{j}^{2}\right)$ defined for every $G \in W_{F_{1}}$ and $G \in W_{F_{2}}$ 
respectively. Suppose that $W_{F_{1}} \cap W_{F_{2}} \neq \emptyset$. We claim that $i_{j}^{1}=i_{j}^{2}$ and $\beta_{G, j}^{1}=\beta_{G, j}^{2}$ for every $G \in W_{F_{1}} \cap W_{F_{2}}$ and $j$. Since the diameter of $W_{F_{1}} \cup W_{F_{2}}$ is smaller than $d_{2}$ we have that $W_{F_{1}} \cup W_{F_{2}} \subset \tilde{W}_{F_{3}}$, for some $F_{3} \in \Omega_{p, n}$. Note that the distance between two maps in $\left\{F_{1}, F_{2}, F_{3}\right\}$ is smaller than $d_{1}$. In particular the combinatorics of their $k_{0}$ th renormalizations are the same, so $i_{j}^{1}=i_{j}^{2}=i_{j}^{3}$ for every $j$. Consider the complex analytic continuation $\left(\beta_{G, j}^{3}, i_{j}\right)$ of $\left(\beta_{F_{3}, j}, i_{j}\right)$ defined for $G \in \tilde{W}_{F_{3}}$. Then $\left(\beta_{F_{1}, j}^{3}, i_{j}\right)$ and $\left(\beta_{F_{1}, j}, i_{j}\right)$ are repelling periodic points with the same combinatorics. Since $F_{1}$ has negative schwarzian derivative, the minimal principle implies that $\beta_{F_{1}, j}^{3}=\beta_{F_{1}, j}$. In an analogous way $\beta_{F_{2}, j}^{3}=\beta_{F_{2}, j}$. The uniqueness of the analytic continuation of a repelling periodic point implies that $\beta_{G, j}^{3}=\beta_{G, j}^{1}$ for $G \in W_{F_{1}}$ and $\beta_{G, j}^{3}=\beta_{G, j}^{2}$ for $G \in W_{F_{2}}$. This concludes the proof of the claim.

In particular the function

$$
G \mapsto\left(\beta_{G, j}, i_{j}\right)
$$

is well defined and complex analytic in $\mathcal{W}=\cup_{F \in \Omega_{p, n}} W_{F}$. There is a small abuse of notation here since $i_{j}$ depends on $G$, but it is a locally constant function.

Fix $G \in W_{F}$. Let $A_{G, j}: \mathbb{C} \times\left\{i_{j}\right\} \rightarrow \mathbb{C} \times\{j\}$ be the affine transformation that maps $\left(\beta_{G, j}, i_{j}\right)$ to $(-1, j)$ and $\left(0, i_{j}\right)$ to $(0, j)$, and $A_{G}: \mathbb{C}_{n} \rightarrow \mathbb{C}_{n}$ as $A_{G}(x, i)=$ $\left(A_{G, i}(x), i\right)$. Let $D^{F, j}$ be the set

$$
\overline{A_{F, j}^{-1}\left(\left\{z \in \mathbb{C}: \operatorname{dist}(z,[-1,1])<\delta_{0} \text { and }|\operatorname{Im}(z)|<\theta_{0}(\operatorname{Re}(z)+1)\right\} \times\{j\}\right)} \text {. }
$$

Since $\bmod (\hat{V} \backslash \hat{U})>\epsilon_{0}$ we have that

$$
D^{F, j} \subset \hat{U}_{j} \subset \mathcal{D}_{D_{\delta_{0}, \theta_{0}}}^{n_{j}}(F)
$$

Moreover, due the complex bounds, reducing $\theta_{0}$ and $\delta_{0}$ we can assume that the interior of the sets in the family

$$
\left\{F^{m}\left(D^{F, j}\right)\right\}_{m<n_{j}}
$$

are pairwise disjoint, and the intersection of the closure of every two of those sets is contained in

$$
\left\{F^{m}\left(\beta_{F, j}, i_{j}\right)\right\}_{m<n_{j}} .
$$

Let $G \in W_{F}$ and define the set $D^{G, j}$ as

$$
\overline{A_{G, j}^{-1}\left(\left\{z \in \mathbb{C}: \operatorname{dist}(z,[-1,1])<\delta_{0} \text { and }|\operatorname{Im}(z)|<\theta_{0}(\operatorname{Re}(z)+1)\right\} \times\{j\}\right)} \text {. }
$$

Reducing the neighbourhood $W_{F}$ of $F$ and $\theta_{0}$ we can assume that

$$
D^{G, j} \subset \mathcal{D}_{D_{\delta_{0}, \theta_{0}}}^{n_{j}}(G),
$$

for every $G \in W_{F}$ and furthermore the interior of the sets in the family

$$
\left\{G^{m}\left(D^{G, j}\right)\right\}_{m<n_{j}}
$$

are pairwise disjoint, and the intersection of the closure of every two of those sets is contained in

$$
\left\{G^{m}\left(\beta_{G, j}, i_{j}\right)\right\}_{m<n_{j}} .
$$

Define the complexification of the renormalization operator

$$
\mathcal{R}: \mathcal{W} \rightarrow \mathcal{B}_{\text {nor }}\left(D_{\delta_{0}, \theta_{0}}\right)
$$

as

$$
\mathcal{R}(G)(x, j)=A_{G, j+1} \circ G^{n_{j}} \circ A_{G, j}^{-1}(x, j)
$$


if $G \in W_{F}$. The operator $\mathcal{R}$ is a compact complex analytic map. From now on denote $U=D_{\delta_{0}, \theta_{0}}$.

Remark 3.1. Let $\tilde{U}$ be a little larger complex open domain that contains $\bar{U}$. Consider the complex analytic transformation

$$
\tilde{\mathcal{R}}: \mathcal{W} \rightarrow \mathcal{B}_{\text {nor }}(\tilde{U})
$$

defined exactly as in (14). Let

$$
i: \mathcal{B}_{\text {nor }}(\tilde{U}) \rightarrow \mathcal{B}_{\text {nor }}(U)
$$

be the compact linear inclusion between these spaces. Then $\mathcal{R}=i \circ \tilde{\mathcal{R}}$, so the complexification of the renormalization operator is a strongly compact operator as defined in [43].

Let $v \in T_{G} \mathcal{B}_{\text {nor }}(U)$. If $z \in U$ and $G^{j}(z) \in U$ for every $j<i$ then $(G+t v)^{i}$ is defined in a neighbourhood of $z$ and we can define

$$
a_{i}(z)=\left.\frac{\partial}{\partial t}(G+t v)^{i}\right|_{t=0}(z)=\sum_{j=0}^{i-1} D G^{i-j-1}\left(G^{j+1}(z)\right) v\left(G^{j}(z)\right) .
$$

Given $F \in \Omega_{p, n}$ and $G \in W_{F}$. For each $v \in T_{G} \mathcal{B}_{\text {nor }}(U)$ and $z \in U_{j}$ we have

$$
\begin{gathered}
\left(D \mathcal{R}_{G} \cdot v\right)(x, j)=\left.\frac{\partial}{\partial t} A_{G+t v, j+1} \circ(G+t v)^{n_{j}} \circ A_{G+t v, j}^{-1}(x, j)\right|_{t=0} \\
=-\frac{\partial_{G} \beta_{G, j+1} \cdot v}{\beta_{G, j+1}} \cdot A_{G, j+1} \circ G^{n_{j}} \circ A_{G, j}^{-1}(x, j) \\
-\frac{1}{\beta_{G, j+1}}\left(a_{n_{j}} \circ A_{G, j}^{-1}(x, j)+\left(\partial_{x} G^{n_{j}}\right) \circ A_{G, j}^{-1}(x, j) \cdot\left(-\partial_{G} \beta_{G, j} \cdot v x, j\right)\right) .
\end{gathered}
$$

Theorem 3. Let $F \in \mathcal{W}$. Then $D_{F} \mathcal{R}\left(T_{F} \mathcal{B}_{\text {nor }}(U)\right)$ is dense in $T_{\mathcal{R} F} \mathcal{B}_{\text {nor }}(U)$.

Proof. The proof is quite similar to the proof of the analogous statement in [3]. Let $w \in T_{\mathcal{R} F} \mathcal{B}_{\text {nor }}(U)$. Then $w(-1, k)=0$ and $w^{\prime}(0, k)=0$ for every $k$. We are going to define a function

$$
\hat{v}: \cup_{j} \cup_{m<n_{j}} G^{m}\left(D^{G, j}\right) \rightarrow \mathbb{C}
$$

in the following way. Define the function $\hat{v}$ as 0 on

$$
\cup_{j} \cup_{0<m<n_{j}} G^{m}\left(D^{G, j}\right),
$$

and

$$
\hat{v}(z)=\left[D G^{n_{j}-1}(G(z))\right]^{-1} \cdot w \circ A_{G, j}(z)
$$

for $z \in D^{G, j}$. Also define $\hat{v}(-1, k)=0$ for every $k$. Then $\hat{v}$ is well defined, it is continuous on

$$
\Lambda=\cup_{j} \cup_{m<n_{j}} G^{m}\left(D^{G, j}\right) \cup\{(-1, k)\}_{k},
$$

and it is complex analytic in the interior of $\Lambda$.

Moreover $\hat{v}$ vanishes on the orbit of the periodic points $\left\{\beta_{G, j}\right\}_{j}$. Since $\mathbb{C} \times\{i\} \backslash \Lambda$ is a connected set, by Mergelyan's Theorem for each given $\epsilon>0$ and $i$ we can find a polynomial $q_{i}$ such that $\left|\hat{v}(z)-q_{i}(z)\right|<\epsilon$ for $z \in \Lambda_{i}=\Lambda \cap \mathbb{C} \times\{i\}$. Define

$$
\hat{q}_{i}(z)=q_{i}(z)-q_{i}^{\prime}(0, i) z-q_{i}(-1, i)-q_{i}^{\prime}(0, i)
$$

Note that $\hat{q}_{i}^{\prime}(0, i)=0$ and $\hat{q}_{i}(-1, i)=0$. Define $q(x, i)=\hat{q}_{i}(x)$. We have that $q \in T_{G} \mathcal{B}_{\text {nor }}(U)$ and

$$
\left|D_{G} \mathcal{R} \cdot q-w\right|_{\mathcal{B}(U)} \rightarrow_{\epsilon \rightarrow 0} 0 .
$$




\section{Action of $D \mathcal{R}$ on horizontal directions.}

4.1. Horizontal direction. Let $F: I_{F}^{n} \rightarrow I_{F}^{n}$ be a real analytic extended map that is either infinitely renormalizable with bounded combinatorics in $\mathcal{C}_{p, n}$ or whose critical points belongs to the same periodic orbit. A continuous function

$$
v: I_{F}^{n} \rightarrow T \mathbb{C}_{n}
$$

is a horizontal direction of $F$ if

1. For each $x \in I_{F}^{n}$ we have $v(x) \in T_{F(x)} \mathbb{C}_{n}$.

2. The function $v$ is real analytic in the interior of $I_{F}^{n}$.

3. There is a quasiconformal vector field

$$
\alpha: W \rightarrow T \mathbb{C}_{n},
$$

defined in a complex neighbourhood $W$ of the post critical set of $F$, such that

$$
v(x)=\alpha(F(x))-D F(x) \cdot \alpha(x)
$$

for every $x$ in the post critical set.

4. We have $\alpha(c)=0$ for every critical point $c$ of $F$.

Denote by $E_{F}^{h}$ the set of $v \in T_{F} \mathcal{B}_{\text {nor }}(U)$ such that $v$ is horizontal. Of course $E_{F}^{h}$ is a linear subspace of $T_{F} \mathcal{B}_{\text {nor }}(U)$.

Proposition 4.1 (Infinitesimal pullback argument. Avila, Lyubich and de Melo $[3])$. Let $F \in \Omega_{n, p}$. Let

$$
F: W \rightarrow V
$$

be a polynomial-like extension of $F$ and $v \in \mathcal{B}(W) \cap T_{F} \mathcal{B}_{\text {nor }}(U)$ such that there exists a quasiconformal vector field $\alpha$, defined in a neighbourhood of the post critical set of $F$, such that

$$
v(x)=\alpha \circ F(x)-D F(x) \cdot \alpha(x)
$$

for every $x \in P(F)$. In particular $v \in E_{F}^{h}$. Reducing a little bit the domain $W$, there exists a quasiconformal vector field extension $\alpha: W \rightarrow \mathbb{C}$ such that (17) holds for every $x \in W$.

Proposition 4.2 (Invariance). Let $F \in \Omega_{n, p}$. Then

$$
\begin{gathered}
D_{F} \mathcal{R}\left(E_{F}^{h}\right) \subset E_{\mathcal{R} F}^{h}, \\
\left(D_{F} \mathcal{R}\right)^{-1}\left(E_{\mathcal{R} F}^{h}\right) \subset E_{F}^{h} .
\end{gathered}
$$

Proof. The proof of (18) is quite similar to the proof of a similar statement in [41]. Indeed, consider $a_{i}$ as in (15). Note that

$$
a_{i}(z)=v\left(F^{i-1}\right)+D F\left(F^{i-1}(z)\right) a_{i-1}(z) .
$$

By an inductive argument one can show that

$$
a_{i}=\alpha \circ F^{i}-D F^{i} \cdot \alpha
$$

on $P(F)$. Denote

$$
\alpha\left(\beta_{F, j+1}\right)=\partial_{F} \beta_{F, j+1} \cdot v
$$


then if $z=(x, j) \in P(F)$ we have

$$
\begin{aligned}
& \left(D \mathcal{R}_{F} \cdot v\right)(x, j) \\
& =-\frac{\partial_{F} \beta_{F, j+1} \cdot v}{\beta_{F, j+1}} \cdot A_{F, j+1} \circ F^{n_{j}} \circ A_{F, j}^{-1}(x, j) \\
& -\frac{1}{\beta_{F, j+1}}\left(a_{n_{j}} \circ A_{F, j}^{-1}(x, j)+\left(D F^{n_{j}}\right) \circ A_{F, j}^{-1}(x, j) \cdot\left(-\partial_{F} \beta_{F, j} \cdot v x, j\right)\right) \\
& =-\frac{\alpha\left(\beta_{F, j+1}\right)}{\beta_{F, j+1}} \cdot(\mathcal{R} F)(z) \\
& -\frac{1}{\beta_{F, j+1}} \alpha \circ A_{F, j+1}^{-1} \circ A_{F, j+1} \circ F^{n_{j}} \circ A_{F, j}^{-1}(x, j)+\frac{\beta_{F, j}}{\beta_{F, j+1}} D F^{n_{j}} \circ A_{F, j}^{-1}(x, j) \cdot \frac{1}{\beta_{F, j}} \alpha \circ A_{F, j}^{-1}(x, j) \\
& +D_{z}(\mathcal{R} F) \cdot\left(\frac{\alpha\left(\beta_{F, j}\right)}{\beta_{F, j}} x, j\right) \\
& =-\frac{\alpha\left(\beta_{F, j+1}\right)}{\beta_{F, j+1}} \cdot(\mathcal{R} F)(z) \\
& -\frac{1}{\beta_{F, j+1}} \alpha \circ A_{F, j+1}^{-1} \circ(\mathcal{R} F)(z)+D_{z}(\mathcal{R} F) \cdot \frac{1}{\beta_{F, j}} \alpha \circ A_{F, j}^{-1}(x, j) \\
& +D_{z}(\mathcal{R} F) \cdot\left(\frac{\alpha\left(\beta_{F, j}\right)}{\beta_{F, j}} x, j\right) .
\end{aligned}
$$

Define the vector field $r(\alpha)$ as

$$
r(\alpha)(x, j)=-\frac{1}{\beta_{F, j}} \alpha \circ A_{F, j}^{-1}(x, j)-\left(\frac{\alpha\left(\beta_{F, j}\right)}{\beta_{F, j}} \cdot x, j\right)
$$

for $z=(x, j) \in U_{j}$. Then

$$
\left(D \mathcal{R}_{F} \cdot v\right)(z)=r(\alpha) \circ(\mathcal{R} F)(z)-D_{z}(\mathcal{R} F) \cdot r(\alpha)(z)
$$

for $z$ in the postcritical set of $\mathcal{R} F$. Note that $r(\alpha)$ is a quasiconformal vector field in a neighbourhood of the post critical set of $\mathcal{R} F$. So $D \mathcal{R}_{F} \cdot v \in E_{\mathcal{R} F}^{h}$.

Now suppose that $v \in\left(D_{F} \mathcal{R}\right)^{-1}\left(E_{\mathcal{R} F}^{h}\right)$. Then $D \mathcal{R}_{F} \cdot v \in E_{\mathcal{R} F}^{h}$, so there exists a quasiconformal vector field $\gamma: \mathbb{C}_{n} \rightarrow \mathbb{C}$ such that

$$
\left(D \mathcal{R}_{F} \cdot v\right)(z)=\gamma \circ(\mathcal{R} F)(z)-D_{z}(\mathcal{R} F) \cdot \gamma(z) .
$$

for every $z$ in a neighbourhood of the post critical set of $\mathcal{R} F$. Define

$$
\delta_{j}=\left.\partial_{t} \beta_{F+t v, j}\right|_{t=0}=\partial_{F} \beta_{F, j} \cdot v
$$

Define $\alpha$ in $A_{F, j}(U)$ as

$$
\alpha(z)=\beta_{F, j} \gamma \circ A_{F, j}^{-1}(z)+\delta_{j} A_{F, j}^{-1}(z) .
$$

Let $z$ be a point very close to the post critical set of $F$. Then

$$
\left\{k \geq 0 \text { s.t. } F^{k}(z) \in \cup_{j \leq n} A_{F, j}(U)\right\} \neq \emptyset
$$

Let $k(z)$ be a minimal element of the above set. Not hat $z \mapsto k(z)$ is locally constant. We define $\alpha(z)$ for $z$ close to the post critical set of $F$ by induction of $k(z)$. We already defined $\alpha(z)$ when $k(z)=0$. If $k(z)>0$ then $k(F(z))=k(z)-1$ and we define

$$
\alpha(z)=\frac{v(z)+\alpha(F(z))}{D F(z)} .
$$


One can check that $\alpha$ is a quasiconformal vector field and

$$
v=\alpha(F(z))-D F(z) \alpha(z)
$$

in a neighbourhood of the post critical set of $F$, so $v \in E_{F}^{h}$.

Proposition 4.3. Let $F \in \Omega_{n, p}$. Then $D \mathcal{R}_{F}$ is injective.

Proof. Let $v$ be such that $D \mathcal{R}_{F} \cdot v=0$. By Proposition 4.2 we have that $v \in E_{F}^{h}$. By Proposition 4.1 there is a quasiconformal vector field $\alpha$, defined in a neighborhood of the Julia set of $F$, satisfying (17) for every $x$ on its Julia set. Let $r(\alpha)$ be the quasiconformal vector field defined by (20). Then by (21) we have that $r(\alpha)$ satisfies

$$
0=r(\alpha) \circ(\mathcal{R} F)(z)-D_{z}(\mathcal{R} F) \cdot r(\alpha)(z) .
$$

for every $z$ in the Julia set of $\mathcal{R} F$. We can easily conclude that $r(\alpha)(z)=0$ at every repelling periodic point $z$ of $\mathcal{R} F$ and consequently at every point of its Julia set. By (20) we have that $\alpha$ is zero at every point of the small Julia sets of $F$ corresponding to this renormalization and, by (17) we have that $v$ vanishes in these small Julia sets as well. So $v=0$ everywhere.

Proposition 4.4 (Closedness). Let $F_{k} \in \Omega_{n, p}$ and $v_{k} \in E_{F_{k}}^{h} \subset T \mathcal{B}_{\text {nor }}(U)$ be sequences such that $\left(F_{k}, v_{k}\right)$ converges to $(F, v) \in \mathcal{B}_{\text {nor }}(U) \times T \mathcal{B}_{\text {nor }}(U)$. Then $F \in \Omega_{p, n}$ and $v \in E_{F}^{h}$. In particular $E_{F}^{h}$ is Banach subspace of $T_{F} \mathcal{B}_{\text {nor }}(U)$.

Proof. Due the definition of the operator $\mathcal{R}$, the map $\mathcal{R} F$ has a polynomial-like extension

$$
\mathcal{R} F: W \rightarrow V,
$$

with $\bar{U} \subset W$. Reducing $V$ a little bit, we can assume $\partial V$ is a finite union of analytic curves and that for $k$ large enough the map

$$
\mathcal{R} F_{k}: W_{k} \rightarrow V,
$$

where $W_{k} \subset \mathbb{C}_{n}, \bar{U} \subset W_{k}$, is the set whose connected components are the connected components of

$$
\left(\mathcal{R} F_{k}\right)^{-1} V
$$

that intersect $\{(0, j)\}_{j}$, is a polynomial-like extension of $\mathcal{R} F_{k}$. Since $v_{k} \in E_{F_{k}}^{h}$ we have that $D_{F_{k}} \mathcal{R} \cdot v_{k} \in E_{\mathcal{R} F_{k}}^{h}$, so there exists a quasiconformal vector field $\tilde{\gamma}^{k}$ such that

$$
\left(D \mathcal{R}_{F_{k}} \cdot v_{k}\right)(z)=\tilde{\gamma}^{k} \circ\left(\mathcal{R} F_{k}\right)(z)-D_{z}\left(\mathcal{R} F_{k}\right) \cdot \tilde{\gamma}^{k}(z) .
$$

holds for $z$ in a neighbourhood of the post critical set of $\mathcal{R} F_{k}$.

Now we use the infinitesimal pullback argument in Avila, Lyubich and de Melo [3]. For each $k$, there exist $C>0$ and a quasiconformal vector field $\gamma_{0}^{k}: \mathbb{C}_{n} \rightarrow \mathbb{C}$ with the following properties

1. The vector field $\gamma_{0}^{k}$ vanishes outside $V$. Moreover $\gamma_{0}^{k}(-1)=\gamma_{0}^{k}(0)=0$.

2. It satisfies

$$
\left(D \mathcal{R}_{F_{k}} \cdot v_{k}\right)(z)=\gamma_{0}^{k} \circ\left(\mathcal{R} F_{k}\right)(z)-D_{z}\left(\mathcal{R} F_{k}\right) \cdot \gamma_{0}^{k}(z) .
$$

for every $z \in \partial W_{k}$.

3. The vector field $\gamma_{0}^{k}$ is $C^{\infty}$ in a neighbourhood of

$$
\overline{V \backslash W_{k}}
$$

and

$$
\left|\bar{\partial} \gamma_{0}^{k}\right| \leq C
$$


on this set.

4. We have $\gamma_{0}^{k}=\tilde{\gamma}^{k}$ in a neighbourhood of the post critical set of $\mathcal{R} F_{k}$. Define by induction $\gamma_{j}^{k}$ as 0 outside $V$ and

$$
\gamma_{j+1}^{k}(z)=\frac{\gamma_{j}^{k} \circ\left(\mathcal{R} F_{k}\right)(z)-\left(D \mathcal{R}_{F_{k}} \cdot v_{k}\right)(z)}{D_{z}\left(\mathcal{R} F_{k}\right)}
$$

on $V \backslash\{(0, m)\}_{m}$, and $\gamma_{j+1}^{k}(0, m)=0$.

Using the McMullen compactness criterion for quasiconformal vectors fields [32, Corollary A.11], one can prove that for each $k$ the sequence

$$
\hat{\gamma}_{j}^{k}=\frac{1}{j} \sum_{t=0}^{j-1} \gamma_{t}^{k}
$$

has a convergent subsequence, uniform on compact subsets of $\mathbb{C}_{n}$. Moreover such limits are quasiconformal vectors fields. Let $\gamma_{\infty}^{k}$ be one of theses limits. Since the filled-in Julia sets of the polynomial-like extensions of $\mathcal{R} F_{k}$ do not support invariant line fields [40] we conclude that $\left|\bar{\partial} \gamma_{\infty}^{k}\right| \leq C$ on $\mathbb{C}_{n}$. Note that

$$
\left(D \mathcal{R}_{F_{k}} \cdot v_{k}\right)(z)=\gamma_{\infty}^{k} \circ\left(\mathcal{R} F_{k}\right)(z)-D_{z}\left(\mathcal{R} F_{k}\right) \cdot \gamma_{\infty}^{k}(z), z \in \bar{U} .
$$

By the compactness criterion for quasiconformal vectors fields in McMullen [32] we can consider a convergent subsequence $\gamma^{k_{t}} \infty \rightarrow_{t} \gamma$, where $\gamma$ is a quasiconformal vector field on $\mathbb{C}_{n}$ and the convergence is uniform on compact subsets of $\mathbb{C}_{n}$. By (23) we have

$$
\left(D \mathcal{R}_{F} \cdot v\right)(z)=\gamma \circ(\mathcal{R} F)(z)-D_{z}(\mathcal{R} F) \cdot \gamma(z), z \in \bar{U},
$$

so $D \mathcal{R}_{F} \cdot v \in E_{\mathcal{R} F}^{h}$, so by (19) we have $v \in E_{F}^{h}$.

Proposition 4.5 (Contraction on the horizontal directions). There exist $K$ and $\theta_{1}>1$ such that for every $F \in \Omega_{n, p}$ and $v \in E_{F}^{h}$ we have

$$
\left|D_{F} \mathcal{R}^{i} \cdot v\right|_{T \mathcal{B}_{\text {nor }}(U)} \leq K \theta_{1}{ }^{-i}|v|_{T \mathcal{B}_{\text {nor }}(U)} .
$$

We do not provide a proof for Proposition 4.5 since it can be proven in exactly the same way it is done in the unimodal setting. One can use the argument by Lyubich [27, Theorem 6.3] using the Schwarz's lemma and the rigidity of McMullen's towers [32]. An infinitesimal argument using the rigidity of McMullen's towers and the compactness of the renormalization operator is given in [41, Proposition 3.9] (in the case of the fixed point of the period doubling renormalization) can be also applied here. We also cite the new methods by Avila and Lyubich [2] to prove the contraction in the horizontal directions in the case of unimodal unbounded combinatorics.

Proposition 4.6 (Contraction on the hybrid classes). There exists $\lambda_{1} \in(0,1)$ with the following property. Let $F$ be a real-analytic polynomial-like map of type $n$ that is infinitely renormalizable with combinatorics bounded by $p$. Then there exist $G \in \Omega_{n, p}$ and $k_{0}=k_{0}(F)$ and $C=C(F)$ such that $\mathcal{R}^{k} F \in \mathcal{B}(U)$ for every $k \geq k_{0}$ and

$$
\left|\mathcal{R}^{k} F-\mathcal{R}^{k} G\right|_{\mathcal{B}_{\text {nor }}(U)} \leq C \lambda_{1}^{k} \text { for } k \geq k_{0} .
$$

Proof. One can prove this in a quite similar way to the proof of the main result in [40]. An alternative proof is obtained using Proposition 4.5 and the same argument as in the proof of Theorem 1 in [41]. 
Next we show that every map in $\Omega_{p, n}$ can be approximated the hyperbolic polynomial-like maps of type $n$.

Proposition 4.7. Let $G \in \mathcal{W}$ be such that there exist domains $\hat{U}$ and $\hat{V}$, whose boundaries are analytic Jordan curves, such that $\bmod \hat{V} \backslash \hat{U}>\epsilon_{0} / 2$ and

$$
G: \hat{U} \rightarrow \hat{V}
$$

is a real polynomial-like map of type $n$ that is infinitely renormalizable with combinatorics bounded by $p$. Then there exist polynomial-like maps of type $n$

$$
G_{i}: \hat{U}^{i} \rightarrow \hat{V}^{i}
$$

such that
A. we have $\bmod \hat{V}^{i} \backslash \hat{U}^{i} \geq \epsilon_{0} / 2$ and $G_{i} \in \mathcal{B}_{\text {nor }}(U)$,
B. all critical points of $G_{i}$ belong to the same periodic orbit,
C. we have

$$
\lim _{i}\left|G_{i}-G\right|_{\mathcal{B}_{\text {nor }}(U)}=0 .
$$

Proof. We will use the notation introduced in [40]. Let $\sigma=\left(\sigma_{1}, \sigma_{2}, \ldots\right)$ be the combinatorics of $G$. By Proposition 2.2 in [40], there exists a sequence of polynomial $P_{i}$ of type $n$ with combinatorics $\sigma_{i} \star \cdots \star \sigma_{1}$. By Corollary 2.3 in [40] any accumulation point of this sequence is a polynomial $P$ of type $n$ that is infinitely renormalizable with combinatorics $\sigma$. By the proof of Theorem 2 in [40] there is only one polynomial of type $n$ with combinatorics $\sigma$, so the sequence $P_{i}$ indeed converges to $P$. Indeed there are now far more general rigidity results for polynomials. See Kozlovski, Shen and van Strien [24][23].

Since $P_{i}$ is a convergent sequence of polynomials of type $n$ with connected Julia sets, it is possible to choose domains $\hat{U}^{i}$ and $\hat{V}^{i}$ such that

- $\inf _{i} \bmod \hat{V}^{i} \backslash \hat{U}^{i}>0$,

- $P_{i}: \hat{U}^{i} \rightarrow \hat{V}^{i}$ is a polynomial-like map of type $n$.

and furthermore for some $K>0$ there are $K$-quasiconformal maps

$$
\phi_{i}: \mathbb{C}_{n} \rightarrow \mathbb{C}_{n}
$$

such that

- $\phi_{i}\left(\hat{U}^{i}\right)=\hat{U}$ and $\phi_{i}\left(\hat{V}^{i}\right)=\hat{V}$,

- $\phi_{i}(\bar{z})=\overline{\phi_{i}(z)}$

- $P_{i}: \hat{U}^{i} \rightarrow \hat{V}^{i}$ is a polynomial-like map of type $n$,

- $G \circ \phi_{i}=\phi_{i} \circ P_{i}$ on $\partial \hat{U}^{i}$.

- The sequence $\phi_{i}$ converges to a $K$-quasiconformal map $\phi$.

- If $\hat{U}^{\infty}=\phi^{-1}(\hat{U})$ and $\hat{V}^{\infty}=\phi^{-1}(\hat{V})$ then $P: \hat{U}^{\infty} \rightarrow \hat{V}^{\infty}$ is a polynomiallike map of type $n$.

Let $\mu_{i}$ be the Beltrami field that coincides with $\mu_{i}=\bar{\partial} \phi_{i} / \partial \phi_{i}$ on $\mathbb{C}_{n} \backslash \hat{U}^{i}$, that is invariant under $P_{i}$, and $\mu_{i}=0$ on $K\left(P_{i}\right)$. Let $\psi_{i}: \mathbb{C}_{n} \rightarrow \mathbb{C}_{n}$ be the unique quasiconformal map such that $\psi_{i}(-1, j)=(-1, j)$ and $\psi_{i}(0, j)=(0, j)$ for every $j$, and $\mu_{i}=\bar{\partial} \psi_{i} / \partial \psi_{i}$ on $\mathbb{C}_{n}$. Define

$$
G_{i}=\psi_{i} \circ P_{i} \circ \psi_{i}^{-1}
$$

Then

$$
G_{i}: \psi_{i}\left(\hat{U}^{i}\right) \rightarrow \psi_{i}\left(\hat{V}^{i}\right)
$$


is a polynomial-like map of type $n$. Note that

$$
\inf _{i} \bmod \psi_{i}\left(\hat{V}^{i}\right) \backslash \psi_{i}\left(\hat{U}^{i}\right)>0 .
$$

Every subsequence of $G_{i}$ has a convergent subsequence. Let $F$ be one these accumulation points. We claim that $F=G$. Note that every accumulation point is of the form $F=\psi \circ P \circ \psi^{-1}$, where $\psi$ is a $K$-quasiconformal map that is an accumulation point of the sequence $\psi_{i}$. We can assume, without loss of generality, that $\phi_{i}$ converges to a $K$-quasiconformal map $\phi$.

Notice that

$$
\phi_{i} \circ \psi_{i}^{-1} \circ G_{i} \circ \psi_{i} \circ \phi_{i}^{-1}(z)=\phi_{i} \circ P_{i} \circ \phi_{i}^{-1}(z)=G(z)
$$

for $z \in \phi_{i}\left(\partial \hat{U}^{i}\right)=\partial \hat{U}$. Taking the limit on $i$ we obtain

$$
\phi \circ \psi^{-1} \circ F \circ \psi \circ \phi^{-1}(z)=G(z)
$$

for $z \in \partial \hat{U}$. Moreover since $\psi_{i} \circ \phi_{i}^{-1}$ is conformal in $\mathbb{C}_{n} \backslash \hat{U}$ we conclude that $\psi \circ \phi^{-1}$ is conformal in $\mathbb{C}_{n} \backslash \hat{U}$. Since $F$ and $G$ are both infinitely renormalizable with the same combinatorics, one can use the Sullivan's pullback argument to conclude that there is quasiconformal conjugacy $H$ between $F: \hat{U}^{\infty} \rightarrow \hat{V}^{\infty}$ and $G: \hat{U} \rightarrow \hat{V}$ such that $H$ is conformal in $\mathbb{C}_{n} \backslash K(F)$. Since there are not invariant line fields supported of $K(F)$ we conclude that $H$ in conformal on $\mathbb{C}_{n}$, so $H$ is affine on each connected component of $\mathbb{C}_{n}$. Since $H(-1, j)=(-1, j)$ and $H(0, j)=(0, j)$ for every $j$, we conclude that $H$ is the identity. So $F=G$ and $G_{i}$ converges to $G$. Itens A., B. and C. of Proposition 4.7 follows easily from this.

4.2. Vertical directions, codimension of $E^{h}$ and vector bundles. Let $f: V_{1} \rightarrow$ $V_{2}$ be a polynomial-like map. Let $\mathbb{B}_{f}$ be the vector space of the germs of holomorphic functions defined in a neighborhood of $K(f)$. We say that $v \in \mathbb{B}_{f}$ is a vertical vector if there exists a holomorphic vector field $\alpha$ defined on $\overline{\mathbb{C}} \backslash K(f)$ such that

$$
v(x)=\alpha \circ f(x)-D f(x) \alpha(x)
$$

for every $x$ close to $K(f)$ and in the domain of $\alpha$, and additionally

$$
\lim _{z \rightarrow 0} z^{2} \alpha(1 / z)=0 .
$$

We have an analogous definition for polynomial-like extended maps of type $n$. Denote the set of vertical directions of $f$ as $\hat{E}_{f}^{v}$. Recall that $v \in \mathbb{B}_{f}$ is a horizontal vector $\left(v \in \hat{E}_{f}^{h}\right)$ if there is quasiconformal vector field on $\mathbb{C}$ such that (25) holds in a neighborhood of $K(f)$ and $\bar{\partial} \alpha=0$ on $K(f)$. Lyubich [27] proved that

$$
\mathbb{B}_{f}=\hat{E}_{f}^{h}+\hat{E}_{f}^{v}
$$

The same statement holds for polynomial-like extended maps of type $n$. Note that if $F \in \mathcal{W}$ has an extension that is a real polynomial-like extended map of type $n$ and $F$ is either infinitely renormalizable with bounded combinatorics in $\mathcal{C}_{p, n}$ or whose critical points belongs to the same periodic orbit then $E_{F}^{h}=\hat{E}_{F}^{h} \cap T \mathcal{B}_{\text {nor }}(U)$. Here $E_{F}^{h}$ is as defined in Section 4.1.

Due the infinitesimal pullback argument, if $f$ does not have invariant line fields on its Julia set $J(f)$ then $\hat{E}_{f}^{h} \cap \hat{E}_{f}^{v}$ is exactly the space of vectors $v$ such that there 
exists a vector field $\alpha(z)=a z+b$ on $\overline{\mathbb{C}}$ that satisfies (25) in a neighborhood of $K(f)$. In particular if $F \in \Omega_{n, p}$ we have

$$
T \mathcal{B}_{\text {nor }}(U)=E_{F}^{h} \oplus E_{F}^{v} .
$$

Proposition 4.8. If $f: V_{1} \rightarrow V_{2}$ is a polynomial-like of degree $d$ generated by the restriction of a polynomial of degree $d$ then $\hat{E}_{f}^{v}$ is exactly the linear space of polynomials of degree $d$. If $f$ is a polynomial-like extended map of type $n$ such that on each $\mathbb{C} \times\{i\}$ the map $f$ coincides with a quadratic polynomial then $\hat{E}_{f}^{v}$ is the space of vectors that coincides with quadratic polynomials on each $\mathbb{C} \times\{i\}$.

Proof. Suppose that $f$ is a polynomial of degree $d$ and let $v \in \hat{E}_{f}^{v}$. Then the r.h.s. of (25) implies that $v$ extends to an entire holomorphic function. Of course (26) implies that

$$
|\alpha(y)| \leq C|y|
$$

for some $C$, provided $y \in \mathbb{C}$ has large modulus. Since $D f$ is a polynomial of degree $d-1$ it follows from (25) that

$$
|v(x)| \leq \tilde{C}|x|^{d},
$$

for some $\tilde{C}$, provided $|x|$ is large. So $v$ is a polynomial whose degree is at most $d$. On the other hand, if $v$ is a polynomial of degree at most $d$ we have that $f_{t}=f+t v$ is a polynomial of degree $d$ for every small $t$. Every $f_{t}$ have the very same external class (see Lyubich [27]). This implies that $v=\left.\partial_{t} f_{t}\right|_{t=0} \in \hat{E}_{f}^{v}$. The proof in the case of a polynomial-like extended map of type $n$ is analogous.

The following is similar to Lyubich [27, Lemma 4.10], but for the sake of completeness we provide details.

Proposition 4.9. Let $F: W \rightarrow V$ be a polynomial-like map of type $n$ with connected Julia set satisfying

i. $0<\epsilon_{0}<\bmod (V \backslash W)<\epsilon_{1}$,

ii. $\operatorname{diam} K(F) \cap(\mathbb{C} \times\{i\}) \geq 1$ for every $i$,

iii. diam $V \cap(\mathbb{C} \times\{i\}) \leq C_{1}$ for every $i$.

Let $v \in E_{F}^{v} \cap \mathcal{B}(W)$. Consider the holomorphic vector field

$$
\alpha: \overline{\mathbb{C}}_{n} \backslash K(F) \rightarrow \mathbb{C}
$$

such that $\lim _{z \rightarrow 0} z^{2} \alpha(1 / z)=0$ and

$$
v(z)=\alpha(F(z))-D F(z) \alpha(z)
$$

for every $z \in W \backslash K(F)$. Then there is $C_{2}>0$, that depends only on $\epsilon_{0}, \epsilon_{1}$ and $C_{1}$, such that

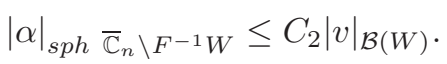

Here $|\cdot|_{\text {sph } Q}$ denotes the sup norm on $Q$ considering the spherical metric on each component of $\mathbb{C}_{n}$.

Proof. Define $K_{i}(F)=K(F) \cap(\mathbb{C} \times\{i\}), 1 \leq 1 \leq n$. Let

$$
\phi_{i}:(\overline{\mathbb{C}} \times\{i\}) \backslash K_{i}(F) \rightarrow \mathbb{D} \times\{i\}
$$

be conformal maps such that $\phi_{i}(\infty, i)=(0, i)$. Define the conformal maps

$$
\phi: \overline{\mathbb{C}}_{n} \backslash K(F) \rightarrow \mathbb{D} \times\{1, \ldots, n\}
$$


as $\phi(z, i)=\phi_{i}(z, i)$. Define

$$
\tilde{W}=\phi(W \backslash K(F)), \tilde{V}=\phi(V \backslash K(F))
$$

and

$$
\tilde{F}: \tilde{W} \rightarrow \tilde{V}
$$

as

$$
\tilde{F}(z, i)=\phi \circ F \circ \phi^{-1}(z, i) .
$$

Let $\psi(x, i)=\left(z /|z|^{2}, i\right), \hat{W}=\overline{\tilde{W} \cup \psi(\tilde{W})}$ and $\hat{V}=\overline{\tilde{V} \cup \psi(\tilde{V})}$. Then $\tilde{F}$ has a analytic extension to a covering

$$
\hat{F}: \hat{W} \rightarrow \hat{V}
$$

satisfying $\hat{F}(\mathbb{S} \times\{1, \ldots, n\})=\mathbb{S} \times\{1, \ldots, n\}$. Note that each connected component of

$$
\hat{V} \backslash \hat{W}
$$

is an annulus with modulus larger than $\epsilon_{0}$. This implies that there is $k$ (that depends only on $\epsilon_{0}, \epsilon_{1}, C_{1}$ and $n$ ) such that

$$
\left|D \hat{F}^{k n}(z, i)\right| \geq 2
$$

for every $(z, i) \in \hat{F}^{-(k n+1)} \hat{W}$. Note that there is $C_{3}>1$, that depends only on $\epsilon_{0}$, $\epsilon_{1}, C_{1}$, such that

- $|D \phi(z, i)| \in\left[1 / C_{3}, C_{3}\right]$ for every $(z, i) \in W \backslash F^{-(k n+1)} W$,

- $|D F(z, i)| \in\left[1 / C_{3}, C_{3}\right]$ for every $(z, i) \in F^{-1} W \backslash F^{-(k n+1)} W$,

- $|D \hat{F}(z, i)| \in\left[1 / C_{3}, C_{3}\right]$ for every $(z, i) \in \hat{F}^{-1} \hat{W} \backslash \hat{F}^{-(k n+1)} \hat{W}$.

- We have $1 / C_{3} \leq|z| \leq C_{3}$ for every $z \in \partial F^{-1} W$.

Define

$$
\hat{\alpha}: \mathbb{D} \times\{1, \ldots, n\} \rightarrow \mathbb{C}
$$

as

$$
\hat{\alpha}(z, i)=D \phi\left(\phi^{-1}(z, i) \alpha\left(\phi^{-1}(z, i)\right)\right.
$$

and

Then $\hat{\alpha}(0)=0$,

$$
\hat{v}(z, i)=D \phi\left(F\left(\phi^{-1}(z, i)\right)\right) v\left(\phi^{-1}(z, i)\right)
$$

$$
\hat{v}=\hat{\alpha} \circ \hat{F}-D \hat{F} \cdot \hat{\alpha}
$$

and consequently

$$
\sum_{j=0}^{n k} D \hat{F}^{n k-j}\left(\hat{F}^{j+1}(z, i)\right) \hat{v}\left(\hat{F}^{j}(z, i)\right)=\hat{\alpha} \circ \hat{F}^{n k}(z, i)-D \hat{F}^{n k}(z, i) \cdot \hat{\alpha}(z, i) .
$$

for $(z, i) \in \partial \hat{F}^{-(k n+1)} \hat{W}$. Let $\left(z_{0}, i_{0}\right)$ be such that

$$
\left|\hat{\alpha}\left(z_{0}, i_{0}\right)\right|=\max _{(z, i) \in \partial \hat{F}^{-(k n+1)} \tilde{W}}|\hat{\alpha}(z, i)| .
$$

Then

$$
\left|\sum_{j=0}^{n k} D \hat{F}^{n k-j}\left(\hat{F}^{j+1}(z, i)\right) \hat{v}\left(\hat{F}^{j}\left(z_{0}, i_{0}\right)\right)\right| \geq 2 \max _{(z, i) \in \partial \hat{F}^{-(k n+1)} \tilde{W}}|\hat{\alpha}(z, i)|-\max _{(z, i) \in \partial \hat{F}^{-1} \tilde{W}}|\hat{\alpha}(z, i)| .
$$

Since $\hat{\alpha}$ is holomorphic the maximum principle implies

$$
\max _{(z, i) \in \partial \hat{F}^{-(k n+1)} \tilde{W}}|\hat{\alpha}(z, i)| \geq \max _{(z, i) \in \partial \hat{F}^{-1} \tilde{W}}|\hat{\alpha}(z, i)|,
$$


So

$$
\max _{(z, i) \in \partial \hat{F}^{-(k n+1)} \tilde{W}}|\hat{\alpha}(z, i)| \leq C_{4} \sup _{(z, i) \in \hat{F}^{-1} \tilde{W} \backslash \hat{F}^{-(k n+1)} \tilde{W}}|\hat{v}(z, i)| .
$$

Here $C_{4}=n k C_{3}^{n k}$. Consequently

$$
\begin{aligned}
\max _{(z, i) \in \partial F^{-1} W}|\alpha(z, i)| & \leq C_{3} \max _{(z, i) \in \partial \hat{F}^{-1} \tilde{W}}|\hat{\alpha}(z, i)| \\
& \leq C_{3} \max _{(z, i) \in \partial \hat{F}^{-(k n+1)} \tilde{W}}|\hat{\alpha}(z, i)| \\
& \leq C_{3} C_{4} \max _{(z, i) \in \hat{F}^{-1} \tilde{W} \backslash \hat{F}^{-(k n+1)} \tilde{W}}|\hat{v}(z, i)| \\
& \leq C_{3}^{2} C_{4} \max _{(z, i) \in F^{-1} W \backslash F-(k n+1) W}|v(z, i)| \\
& \leq C_{3}^{2} C_{4} \max _{(z, i) \in F^{-1} W}|v(z, i)| .
\end{aligned}
$$

Note that

$$
\begin{aligned}
\sup _{(z, i) \in \overline{\mathbb{C}}_{n} \backslash F^{-1} W}|\alpha(z, i)|_{\text {sph } \overline{\mathbb{C}}} & =\sup _{(z, i) \in \overline{\mathbb{C}}_{n} \backslash F^{-1} W} \frac{2|\alpha(z, i)|}{1+|z|^{2}} \\
& \leq \sup _{(z, i) \in \overline{\mathbb{C}}_{n} \backslash F^{-1} W}\left|\frac{2 \alpha(z, i)}{z^{2}}\right| \\
& \leq \max _{(z, i) \in \partial F^{-1} W}\left|\frac{2 \alpha(z, i)}{z^{2}}\right| \\
& \leq C_{3}^{2} \max _{(z, i) \in \partial F^{-1} W}|\alpha(z, i)|
\end{aligned}
$$

Proposition 4.10 (Codimension of $E_{G}^{h}$ ). For every $G \in \Omega_{n, p}$ the codimension of $E_{G}^{h}$ is $n$.

Proof. By Proposition 4.7 for every $G \in \Omega_{n, p}$ one can find a polynomial-like extension $G: \tilde{U} \rightarrow \tilde{V}$ of type $n$ and a sequence of polynomial-like maps $G_{i}: \hat{U}^{i} \rightarrow \hat{V}^{i}$ of type $n$ whose periodic points belongs to the same critical orbit and such that $U$ is compactly contained in $\hat{U}^{i}$ and $\hat{U}^{i}$ is compactly contained in $\tilde{U}$. Denote $E_{G_{i}}^{j}(\tilde{U})=\hat{E}_{G_{i}}^{j} \cap T \mathcal{B}_{\text {nor }}(\tilde{U})$, where $j \in\{v, h\}$. We claim that $\operatorname{codim} E_{G_{i}}^{h}(\tilde{U})=n$.

Indeed for each $q \in C\left(G_{i}\right)$, let $m_{q}^{i}>0$ be such that $G_{i}^{m_{q}^{i}}(q) \in C\left(G_{i}\right)$ and $G_{i}^{k}(q) \notin$ $C\left(G_{i}\right)$ for every $0<k<m_{q}^{i}$. Given $v \in T \mathcal{B}_{\text {nor }}(U)$, let $v_{q, k}=v\left(G_{i}^{k}(q)\right)$. Then there is a unique solution $\left\{\alpha_{q, k}\right\}_{q \in C\left(G_{i}\right), 1 \leq k \leq m_{q}^{i}}$ for the homogeneous system of linear equations

$$
\begin{gathered}
v_{q, k}=\alpha_{q, k+1}-D G_{i}\left(G_{i}^{k}(q)\right) \cdot \alpha_{q, k}, \quad 1 \leq k<m_{q}^{i}, q \in C\left(G_{i}\right), \\
v_{q, 0}=\alpha_{q, 1}, q \in C\left(G_{i}\right) .
\end{gathered}
$$

In particular

$$
\left(v_{q, k}\right)_{q \in C\left(G_{i}\right), 0 \leq k<m_{q}^{i}} \mapsto\left(\alpha_{q, k}\right)_{q \in C\left(G_{i}\right), 1 \leq k \leq m_{q}^{i}}
$$

is a linear bijection. Note that $v \in E_{G_{i}}^{h}$ if and only if $\alpha_{q, m_{q}^{i}}=0$ for every $q \in C\left(G_{i}\right)$, that is, if and only if $v$ belongs to the kernel of the linear map

$$
v \mapsto\left(\alpha_{q, m_{q}^{i}}\right)_{q \in C\left(G_{i}\right)} .
$$


Since

$$
v \mapsto\left(v_{q, k}\right)_{q \in C\left(G_{i}\right), 0 \leq k<m_{q}^{i}}
$$

and

$$
\left(v_{q, k}\right)_{q \in C\left(G_{i}\right), 0 \leq k<m_{q}^{i}} \mapsto\left(\alpha_{q, m_{q}^{i}}\right)_{q \in C\left(G_{i}\right)}
$$

are onto continuous linear maps it follows that codim $E_{G_{i}}^{h}(\tilde{U})=n$ (Recall that $G_{i}$ has $n$ critical points). So $\operatorname{dim} E_{G_{i}}^{v}(\tilde{U})=n$. Since $\tilde{U}$ is compatible with $G$, we have that [42, Propositon 10.4] implies $\operatorname{dim} E_{G}^{v}(\tilde{U})=\operatorname{codim} E_{G}^{h}(\tilde{U})=n$.

Unfortunatelly $U$ is not compatible with $G$ (the repelling fixed point -1 of $G$ does not belong to the interior of $U$ ), so we need to be a little more careful to conclude that $\operatorname{codim} E_{G}^{h}=n$. Consider the natural affine inclusion

$$
\pi: \mathcal{B}_{\text {nor }}(\tilde{U}) \rightarrow \mathcal{B}_{\text {nor }}(U)
$$

Then $D \pi$ is continuous, injective map, it has dense image and moreover

$$
(D \pi)^{-1} E_{G}^{h}=E_{G}^{h}(\tilde{U}) .
$$

Note that if $\operatorname{codim} E_{G}^{h} \geq k$ then there is a bounded linear onto map $\psi: T_{G} \mathcal{B}_{\text {nor }}(U) \rightarrow$ $\mathbb{R}^{k}$ such that $E_{G}^{h} \subset \operatorname{Ker} \psi$. Since $D \pi$ have dense image we have that $\psi \circ D \pi$ is also a bounded linear onto map such that $E_{G}^{h}(\tilde{U}) \subset \operatorname{Ker} \psi \circ D \pi$, so $\operatorname{codim} E_{G}^{h}(\tilde{U}) \geq$ codim $\operatorname{Ker} \psi \circ D \pi=k$. So codim $E_{G}^{h} \leq \operatorname{codim} E_{G}^{h}(\tilde{U})=n$.

On the other hand, since $\pi$ is injective we have that $\operatorname{dim} \pi\left(E_{G}^{v}(\tilde{U})\right)=n$. If $v \in \pi\left(E_{G}^{v}(\tilde{U})\right) \cap E_{G}^{h}$ then $v \in E_{G}^{v}(\tilde{U}) \cap E_{G}^{h}(\tilde{U})=\{0\}$. So codim $E_{G}^{h} \geq n$.

The following lemma is elementary. We included it here for the sake of completeness.

Lemma 4.11. Let $\left(\mathcal{B}_{i},|\cdot|{ }_{i}\right), i=1,2$, be Banach spaces. Let $\Omega$ be a compact metric space and suppose that for every $f \in \Omega$ we associate vector subspaces $E_{f}^{v} \subset \mathcal{B}_{2} \subset \mathcal{B}_{1}$, $E_{f, i}^{h} \subset \mathcal{B}_{i}$ satisfying

A. For every $f \in \Omega$ we have $\mathcal{B}_{2}=E_{f}^{v} \oplus E_{f, 2}^{h}$ and $E_{f}^{v} \cap E_{f, 1}^{h}=\{0\}$.

B. The set

$$
\left\{(f, v): f \in \Omega, v \in E_{f, i}^{h}\right\}
$$

is a closed subset of $\Omega \times \mathcal{B}_{i}$.

C. We have that

$$
\left\{(f, v): f \in \Omega, v \in E_{f}^{v},|v|_{i} \leq 1\right\}
$$

is a compact subset of $\Omega \times \mathcal{B}_{i}, i=1,2$.

D. There exists $n \in \mathbb{N}$ such that $\operatorname{dim} E_{f}^{v}=n$ for every $f \in \Omega$.

E. The inclusion $\imath: \mathcal{B}_{2} \rightarrow \mathcal{B}_{1}$ is a compact linear operator and

$$
\imath^{-1}\left(E_{f, 1}^{h}\right)=E_{f, 2}^{h} \text {. }
$$

Then

I. The set

$$
E^{v}=\left\{(f, v): f \in \Omega, v \in E_{f}^{v}\right\},
$$

with the topology induced by $\Omega \times \mathcal{B}_{2}$, is a topological vector bundle (with the obvious linear structure on the fibers $E_{f}^{v}$ ) with fibers of dimension $n$. 
II. Let $\sim$ be the equivalent relation on $\Omega \times \mathcal{B}_{2}$ defined by $(f, v) \sim(g, w)$ if and only if $f=g$ and $v-w \in E_{f}^{h}$. Then the quotient topological space

$$
E=\left\{(f,[v]) \text { s.t. } f \in \Omega \text { and }[v] \in \mathcal{B} / E_{f, 2}^{h}\right\} .
$$

is a topological vector bundle with fibers of dimension $n$.

III. Define

$$
|(f,[v])|=\operatorname{dist}_{\mathcal{B}_{2}}\left(v, E_{f, 2}^{h}\right)=\inf \left\{|v-w|_{2}: \quad w \in E_{f, 2}^{h}\right\} .
$$

then

$$
(f,[v]) \in E \mapsto|(f,[v])|_{2}
$$

is continuous.

Proof of I. All limits in the proof of I. and II are in the topology of $\left(\mathcal{B}_{2},|\cdot|_{2}\right)$. Let $u \in \mathcal{B}_{2}$. Then for every $g \in \Omega$ there are unique vectors $v^{g, u} \in E_{g}^{v}$ and $w^{g, u} \in E_{g, 2}^{h}$ such that

First note that

$$
u=v^{g, u}+w^{g, u} .
$$

$$
\sup \left\{\left|v^{g, u}\right|_{2}, g \in \Omega,|u|_{2} \leq 1\right\} \cup\left\{\left|w^{g, u}\right|_{2}, g \in \Omega,|u|_{2} \leq 1\right\}<\infty .
$$

Otherwise there is a sequence $g_{i} \in \Omega,\left|u_{i}\right| \leq 1$ such that

$$
r_{i}=\max \left\{\left|v^{g_{i}, u_{i}}\right|_{2},\left|w^{g_{i}, u_{i}}\right|_{2}\right\} \rightarrow_{i} \infty .
$$

Since $\Omega$ is compact, without loss of generality we can assume that

$$
\lim _{i} g_{i}=g \in \Omega
$$

and C. implies that we can assume

$$
\lim _{i} \frac{v^{g_{i}, u_{i}}}{r_{i}}=v \in E_{g}^{v}
$$

and consequently

$$
\lim _{i} \frac{w^{g_{i}, u_{i}}}{r_{i}}=-v
$$

On the other hand by (29) and B. we have $v \in E_{g}^{h}$, so by A. we conclude $v=0$. This is a contradiction with the definition of $r_{i}$. So (28) holds. We claim that the map

defined by

$$
S: \Omega \times \mathcal{B}_{2} \rightarrow \Omega \times \mathcal{B}_{2}
$$

$$
S(g, u)=\left(g, v^{g, u}\right) \in \mathcal{B}_{2}
$$

is a continuous linear map. Indeed suppose $\lim _{i} g_{i}=g$ and $\lim _{i} u_{i}=u$. By assumption C. and (28), taking a subsequence we may assume that

$$
\lim _{i} v^{g_{i}, u_{i}}=v \in E_{g}^{v}
$$

and consequently by B.

$$
\lim _{i} w^{g_{i}, u_{i}}=\lim _{i} u_{i}-\lim _{i} v^{g_{i}, u_{i}}=u-v \in E_{g, 2}^{h}
$$

By A. we conclude that $v=v^{g, u}$ and $u-v=w^{g, u}$. This proves the claim. Let $f \in \Omega$ and choose a basis $v_{1}, \ldots, v_{n} \in E_{f}^{v}$. Let $v_{i}^{g}=S\left(g, v_{i}\right) \in E_{g}^{v}$ and $w_{i}^{g}=v_{i}-S\left(g, v_{i}\right) \in E_{g, 2}^{h}$, with $g \in \Omega$. Of course

$$
v_{i}=v_{i}^{g}+w_{i}^{g} .
$$


So for every $i$ we have that $g \mapsto v_{i}^{g}$ is continuous and moreover $v_{i}^{f}=v_{i}$ and $w_{i}^{f}=0$. In particular there exists an open neighborhood $O_{1}$ of $f$ in $\Omega$ such that $\left\{v_{i}^{g}\right\}_{i}$ is a basis of $E_{g}^{v}$ for every $g \in O$.

Reducing the neighborhood $O$, we may assume that $E_{g, 2}^{h} \cap E_{f}^{v}=\{0\}$ for every $g \in O$. Otherwise it would exists a sequence $g_{i} \rightarrow_{i} f$ and $w_{i} \in E_{g_{i}, 2}^{h} \cap E_{f}^{v}$ satisfying $\left|w_{i}\right|=1$. By D. we may assume $\lim _{i} w_{i}=w \in E_{f}^{v}$. By B. we have $w \in E_{f, 2}^{h}$, which contradicts A.

Define the map

$$
H: O \times E_{f}^{v} \rightarrow\left\{(g, v): g \in O, v \in E_{g}^{v}\right\}
$$

as

$$
H\left(g, \sum_{i} c_{i} v_{i}\right)=\left(g, \sum_{i} c_{i} v_{i}^{g}\right) .
$$

We have that $H$ is a continuous and bijective map. Note that

$$
H^{-1}(g, u)=(g, v)
$$

where $v$ is the only vector in $E_{f, 2}^{h}$ such that $v-u \in E_{g, 2}^{h}$.

We claim that $H^{-1}$ is continuous. Indeed, suppose that $\lim _{i}\left(g_{i}, u_{i}\right)=(g, u)$, with $g, g_{i} \in O$ and $u, u_{i} \in E_{g_{i}}^{v}$. If $H^{-1}\left(g_{i}, u_{i}\right)=\left(g_{i}, v_{i}\right)$ then $w_{i}=v_{i}-u_{i} \in E_{g_{i}, 2}^{h}$ and $v_{i} \in E_{f}^{v}$. Let $r_{i}=\max \left\{\left|v_{i}\right|_{2},\left|w_{i}\right|_{2}\right\}$. We claim that

$$
\sup _{i} r_{i}<\infty \text {. }
$$

Otherwise without loss of generality we may assume $\lim _{i} r_{i}=\infty$. By D. we may assume that $\lim _{i} v_{i} / r_{i}=\tilde{v} \in E_{f}^{v}$ and consequently $\lim _{i} w_{i} / r_{i}=\tilde{v}$. By B. we have $\tilde{v} \in E_{g, 2}^{h}$. So $\tilde{v}=0$, in contradiction with the definition of $r_{i}$. This proves (30). In particular without loss of generality we can assume $\lim _{i} v_{i}=\hat{v} \in E_{f}^{v}$ and consequently by B. we have $\lim _{i} w_{i}=\hat{w}=\hat{v}-u \in E_{f, 2}^{h}$. In particular $H^{-1}(g, u)=(g, \hat{v})$. So $H^{-1}$ is continuous. So

$$
\hat{H}: O \times \mathbb{R}^{n} \rightarrow\left\{(g, v): g \in O, v \in E_{g}^{v}\right\}
$$

defined by

$$
\hat{H}\left(g,\left(c_{i}\right)_{i}\right)=\left(g, \sum_{i} c_{i} v_{i}^{g}\right)
$$

is a local trivialization of the vector bundle $E^{v}$ in the open set $\{(g, v): g \in O, v \in$ $\left.E_{g}^{v}\right\}$.

Proof of II. Let $\pi: \Omega \times \mathcal{B}_{2} \rightarrow E$ be a natural projection

$$
(f, v) \mapsto\left(f,[v]_{f}\right),
$$

where $[v]_{f}$ represents the equivalent class of $v$ in $\mathcal{B} / E_{f, 2}^{h}$. We will define a homeomorphism

$$
T: E^{v} \rightarrow E
$$

that is a vector bundle homeomorphism. Indeed let $T$ be the restriction of $\pi$ to $E^{v}$. Of course $T$ is a continuous map that preserves the linear structure in the fibers. It is also a bijection, since $T(f, v)=T(g, w)$ implies $f=g$, with $v, w \in E_{f}^{v}$ and $v-w \in E_{f, 2}^{h}$, so by A. we have $v=w$. Note that $T^{-1}\left(f,[u]_{f}\right)=(f, v)$, where $v$ is the unique vector that satisfies $v \in E_{f}^{v}$ and $u-v \in E_{f, 2}^{h}$. Note that 
$T^{-1}\left(f,[u]_{f}\right)=S(f, u)$, where $S$ was defined in the proof of I. Consequently $T^{-1}$ is continuous, since $S$ descends to the quotient space $E$ as $T^{-1}$.

Proof of III. We claim that

$$
(f, v) \in \Omega \times \mathcal{B}_{2} \rightarrow \operatorname{dist}_{\mathcal{B}_{2}}\left(v, E_{f}^{h}\right)
$$

is continuous. Indeed suppose that $\lim _{k}\left(f_{k}, v_{k}\right)=(f, v)$. We have

$$
\left|\operatorname{dist}_{\mathcal{B}_{2}}\left(v_{k}, E_{f_{k}, 2}^{h}\right)-\operatorname{dist}_{\mathcal{B}_{2}}\left(v, E_{f_{k}, 2}^{h}\right)\right| \leq \operatorname{dist}_{\mathcal{B}_{2}}\left(v_{k}-v, E_{f_{k}, 2}^{h}\right) \leq\left|v-v_{k}\right|_{2} \rightarrow_{k} 0,
$$

in particular

$$
\lim _{k} \operatorname{dist}_{\mathcal{B}_{2}}\left(v_{k}, E_{f_{k}, 2}^{h}\right)-\operatorname{dist}_{\mathcal{B}_{2}}\left(v, E_{f_{k}, 2}^{h}\right)=0,
$$

so to prove the claim it is enough to show that

$$
\lim _{k} \operatorname{dist}_{\mathcal{B}_{2}}\left(v, E_{f_{k}, 2}^{h}\right)=\operatorname{dist}_{\mathcal{B}_{2}}\left(v, E_{f, 2}^{h}\right) \text {. }
$$

Fix $\epsilon>0$ and let $w \in E_{f, 2}^{h}$ be such that

$$
|v-w|_{2}<\operatorname{dist}_{\mathcal{B}_{2}}\left(v, E_{f, 2}^{h}\right)+\epsilon .
$$

Then $\lim _{k} S\left(f_{k}, w\right)=0$, where $S$ is as defined in the proof of I. In particular $w_{k}=w-S\left(f_{k}, w\right) \in E_{f_{k}, 2}^{h}$ and for large $k$ we have $\left|v-w_{k}\right|<\operatorname{dist}_{\mathcal{B}_{2}}\left(v, E_{f, 2}^{h}\right)+2 \epsilon$. Since $\epsilon>0$ is arbitrary

$$
\limsup _{k} \operatorname{dist}_{\mathcal{B}_{2}}\left(v, E_{f_{k}, 2}^{h}\right) \leq \operatorname{dist}_{\mathcal{B}_{2}}\left(v, E_{f, 2}^{h}\right) .
$$

On the other hand, if

$$
\liminf _{k} \operatorname{dist}_{\mathcal{B}_{2}}\left(v, E_{f_{k}, 2}^{h}\right) \leq \operatorname{dist}_{\mathcal{B}_{2}}\left(v, E_{f, 2}^{h}\right)-2 \epsilon
$$

then we can assume (taking a subsequence) that there is $w_{k} \in E_{f_{k}, 2}^{h}$ such that

$$
\left|v-w_{k}\right|_{2} \leq \operatorname{dist}_{\mathcal{B}_{2}}\left(v, E_{f, 2}^{h}\right)-\epsilon .
$$

Let $u_{k}=S\left(f, w_{k}\right) \in E_{f, 2}^{v}$. Note that $\sup _{k}\left|w_{k}\right|_{2}<\infty$, which implies $\sup _{k}\left|u_{k}\right|_{2}<$ $\infty$ and consequently $y_{k}=w_{k}-u_{k} \in E_{f, 2}^{h}$ satisfies

$$
\sup _{k}\left|y_{k}\right|_{2}<\infty \text {. }
$$

By B. and E. we can find $y \in E_{f, 1}^{h}$ and a subsequence of $y_{k}$ such that $y_{k}$ converges to $y$ in $\mathcal{B}_{1}$. Taking a subsequence we can assume that $\lim _{k} u_{k}=u \in E_{f}^{v}$ (in the topologies of $\left.\mathcal{B}_{i}, i=1,2\right)$. So $w_{k}$ converges to $u+y$ in $\mathcal{B}_{1}$. By A., B. and E. we have $u=0$. We conclude that

$$
\operatorname{dist}_{\mathcal{B}_{2}}\left(v, E_{f, 2}^{h}\right) \leq \liminf _{k}\left|v-y_{k}\right|_{2} \leq \operatorname{dist}_{\mathcal{B}_{2}}\left(v, E_{f, 2}^{h}\right)-\epsilon,
$$

which is a contradiction. So (32) holds. This proves the claim. Since $(f, v) \sim(g, \tilde{v})$ implies $\operatorname{dist}_{\mathcal{B}_{2}}\left(v, E_{f}^{h}\right)=\operatorname{dist}_{\mathcal{B}_{2}}\left(\tilde{v}, E_{g}^{h}\right)$ the function (31) descends to the quotient topological space $E$ as a continuous function.

Proposition 4.4 implies that

$$
\left\{(F, v), F \in \Omega_{n, p} \text { and } v \in E_{F}^{h}\right\}
$$

is a closed subset of $\Omega_{n, p} \times T \mathcal{B}_{n o r}(U)$. We also have 
Lemma 4.12. The set

$$
\mathcal{E}=\left\{(F, v), F \in \Omega_{n, p} \text { and } v \in E_{F}^{v},|v|_{T \mathcal{B}_{n o r}(U)} \leq 1\right\}
$$

is a compact subset of $\Omega_{n, p} \times T \mathcal{B}_{\text {nor }}(U)$.

Proof. Let $\left(F_{k}, v_{k}\right)$ be a sequence in the set $\mathcal{E}$. Due the complex bounds there exist domains $W_{k}, V_{k} \in \mathbb{C}_{n}$ such that $\overline{W_{k}} \subset V_{k}$ and

$$
F_{k}: W_{k} \rightarrow V_{k}
$$

are polynomial-like maps of type $n$ satisfying

$$
\bmod \left(V_{k} \backslash W_{k}\right) \geq \epsilon_{0} .
$$

Using the same argument as McMullen [31, Theorem 5.8] there is a polynomial-like map of type $n F: W \rightarrow V$ with $\bmod (V \backslash W) \geq \epsilon_{0}$ such that $\lim _{k} F_{k}=F$ in the topology defined by McMullen. In particular $\lim _{k} W_{k}=W$ and $\lim _{k} V_{k}=V$ in the Carathéodory topology and $F_{k}$ converges to $F$ uniformly on compact subsets of $W$. Consequently $\lim _{k} F_{k}=F$ in $\mathcal{B}_{\text {nor }}(U)$ and we can find $\tilde{V}$ compactly contained in $V$ such that

$$
F_{k}: F_{k}^{-1} \tilde{V} \rightarrow \tilde{V}
$$

are polynomial-like maps of type $n$ satisfying

$$
\bmod \left(\tilde{V} \backslash F_{k}^{-1} \tilde{V}\right) \geq \epsilon_{0} / 2
$$

Let $\tilde{W}=F^{-1} \tilde{V}$. Since $v_{k} \in E_{F_{k}}^{v}$ there exist holomorphic vectors fields

$$
\alpha_{k}: \overline{\mathbb{C}}_{n} \backslash K\left(F_{k}\right) \rightarrow \mathbb{C}
$$

such that $\alpha_{k}(\infty)=0$ and

$$
v_{k}(z)=\alpha_{k}\left(F_{k}(z)\right)-D F_{k}(z) \alpha_{k}(z)
$$

for every $z \in W_{k} \backslash K\left(F_{k}\right)$.

Finally, for every large $j>0$ it is possible to find a domain $V^{j}$ such that

$$
K(F) \subset V^{j} \subset \overline{\left\{z \in \mathbb{C}_{n}: \operatorname{dist}(z, K(F))<1 / j\right\}} \subset \tilde{V}
$$

and

$$
F: F^{-1} V^{j} \rightarrow V^{j}
$$

is a polynomial-like map of type $n$. Consequently there is $k_{0}=k_{0}(j)$ such that for every $k \geq k_{0}$ we have that

$$
F_{k}: F_{k}^{-1} V^{j} \rightarrow V^{j}
$$

is a polynomial-like map of type $n$. Note that $F_{k}^{-1} V^{j} \subset \tilde{W}$ for large $k$. Due Proposition 4.9 we have that

$$
\left|\alpha_{k}\right|_{s p h} \overline{\mathbb{C}}_{n} \backslash F_{k}^{-2} V^{j} \leq C_{j}\left|v_{k}\right|_{\mathcal{B}\left(V^{j}\right)} \leq C_{j}\left|v_{k}\right|_{\mathcal{B}(\tilde{W})} .
$$

for large $k$. We claim that

$$
\sup _{k}\left|v_{k}\right|_{\mathcal{B}(\tilde{W})}<\infty
$$

Indeed, otherwise we may assume that $r_{k}=\left|v_{k}\right|_{\mathcal{B}(\tilde{W})}$ diverges to infinity. Then $\hat{\alpha}_{k}=\alpha_{k} / r_{k}$ satisfies

$$
\left|\hat{\alpha}_{k}\right|_{s p h} \overline{\mathbb{C}}_{n} \backslash F_{k}^{-2} V^{j} \leq C_{j}
$$

This implies that a subsequence of $\hat{\alpha}_{k}$ converges uniformly on compact subsets of $\mathbb{C}_{n} \backslash K(F)$ to a holomorphic vector field $\hat{\alpha}: \overline{\mathbb{C}}_{n} \backslash K(F) \rightarrow \mathbb{C}$ and the corresponding 
subsequence of $\hat{v}_{k}=v_{k} / r_{k}$ converges uniformly on compact subsets of $W \backslash K(F)$ to

$$
\hat{v}=\hat{\alpha} \circ F-D F \cdot \hat{\alpha} .
$$

By the maximum principle we have that $\hat{v}_{k}$ is a uniform Cauchy sequence on compact subsets of $W$, so $\hat{v}$ extends to a holomorphic function on $W$ and $\lim _{k} \hat{v}_{k}=\hat{v}$ uniformly on compact subsets of $W$. Since $\left|\hat{v}_{k}\right|_{\mathcal{B}(\tilde{W})}=1$ for every $k$ we have $|\hat{v}|_{\mathcal{B}(\tilde{W})}=1$. On the other hand

$$
|\hat{v}|_{\mathcal{B}(U)}=\lim _{k}\left|\hat{v}_{k}\right|_{\mathcal{B}(U)}=\lim _{k} 1 / r_{k}=0
$$

so $\hat{v}=0$ everywhere, in contradiction with $|\hat{v}|_{\mathcal{B}(\tilde{W})}=1$. This proves the claim. Now we can use the same argument as in the previous paragraph to conclude that there is a subsequence of $\alpha_{k}$ that converges uniformly on compact subsets of $\mathbb{C}_{n} \backslash K(F)$ to a holomorphic vector field $\alpha: \overline{\mathbb{C}}_{n} \backslash K(F) \rightarrow \mathbb{C}$ and the corresponding subsequence of $v_{k}$ converges in $\mathcal{B}(\tilde{W})$ (and in particular in $\mathcal{B}(U)$ ) so a vector $v$ that satisfies

$$
v=\alpha \circ F-D f \cdot \alpha
$$

on $W \backslash K(F)$. This concludes the proof.

As an immediate consequence of Lemmas 4.11 and 4.12 we have

Proposition 4.13. The quotient topological space

$$
E=\left\{(F,[v]) \text { s.t. } F \in \Omega_{n, p} \text { and }[v] \in T \mathcal{B}_{n o r}(U) / E_{F}^{h}\right\} .
$$

is a topological vector bundle with fibers of dimension $n$. Moreover

$$
(F,[v]) \mapsto|(F,[v])|=\operatorname{dist}_{T \mathcal{B}_{\text {nor }}(U)}\left(v, E_{F}^{h}\right)
$$

is a continuous function.

Proof. Let $\hat{U}$ be a symmetric domain with respect to the real trace of $\mathbb{C}_{n}$, that is compactly contained in the interior of $U$, such that the $\hat{U} \cap \mathbb{R}$ is an interval that contain in its interior the convex closure of the postcritical set of every $F \in \Omega_{n, p}$. Let $\mathcal{B}_{2}=T \mathcal{B}_{\text {nor }}(U), \mathcal{B}_{1}=\mathcal{B}(\hat{U}), E_{F, 2}^{h}=E_{F}^{h}$ and $E_{F, 1}^{h}$ be the set of horizontal vectors of $\mathcal{B}(\hat{U})$. Apply Lemma 4.11.

$$
\text { 5. Hyperbolicity of the } \omega \text {-Limit Set } \Omega_{n, p} \text { OF } \mathcal{R} \text {. }
$$

Given $F \in \Omega_{n, p}$, denote

$$
\mathfrak{B}_{+}(F)=\left\{v \in T_{F} \mathcal{B}_{\text {nor }}(U) \text { s.t. } \sup _{i \in \mathbb{N}}\left|D \mathcal{R}_{f}^{i} \cdot v_{i}\right|<\infty\right\} .
$$

Recall we choose $U=D_{\delta_{0}, \theta_{0}}$. The goal of this section is to prove

Proposition 5.1. Suppose that for every $F \in \Omega_{n, p}$ we have

$$
\mathfrak{B}_{+}(F) \subset E_{F}^{h} \text {. }
$$

Then $\Omega_{n, p}$ is a hyperbolic set. Moreover its stable direction is exactly $E^{h}$.

Proposition 5.1 reduces the study of the hyperbolicity of $\Omega_{n, p}$ to the study of the existence and regularity of the solutions $\alpha$ of the cohomological equation (16). So to show that $\Omega_{n, p}$ is a hyperbolic set it remains to prove

Theorem 4 (Key Lemma). If $F \in \Omega_{n, p}$ then

$$
\mathfrak{B}_{+}(F) \subset E_{F}^{h} .
$$


We will prove Theorem 4 in Section 8. As an immediate consequence of Proposition 5.1 and Theorem 4 we have

Theorem 5 (Theorem B). $\Omega_{n, p}$ is a hyperbolic set. Moreover its stable direction is exactly $E^{h}$.

5.1. A criterium for hyperbolicity of cocycles. Let $E$ be a topological vector bundle with base $\Omega$ and fiber $\mathbb{R}^{n}$ and projection $p: E \rightarrow \Omega$. We denote elements of $E$ by $(x, v)$, where $x \in \Omega$ and $v \in p^{-1}(x)$. We also assume that $\Omega$ is compact. Additionally, assume that $|\cdot|$ is a continuous function

$$
(x, v) \in E \mapsto|(x, v)| \in \mathbb{R}
$$

so that $|\cdot|$ is a norm on each fiber $p^{-1}(x)$. We will abuse the notation writing $|v|$ instead of $(x, v)$.

Let $L: E \rightarrow E$ be a fiber-preserving homeomorphism that is linear on the fibers. The map $L$ is called a linear cocycle on $E$. Define

$$
\begin{aligned}
& \mathfrak{B}_{+}=\left\{(x, v) \in E \text { s.t. } \sup _{i \in \mathbb{N}}\left|v_{i}\right|<\infty, \text { where } L^{i}(x, v)=\left(x_{i}, v_{i}\right)\right\} . \\
& \mathfrak{B}=\left\{(x, v) \in E \text { s.t. } \sup _{i \in \mathbb{Z}}\left|v_{i}\right|<\infty \text {, where } L^{i}(x, v)=\left(x_{i}, v_{i}\right)\right\} . \\
& \mathcal{S}=\left\{(x, v) \in E \text { s.t. } \lim _{i \rightarrow+\infty}\left|v_{i}\right|=0 \text {, where } L^{i}(x, v)=\left(x_{i}, v_{i}\right)\right\} . \\
& \mathcal{U}=\left\{(x, v) \in E \text { s.t. } \lim _{i \rightarrow-\infty}\left|v_{i}\right|=0, \text { where } L^{i}(x, v)=\left(x_{i}, v_{i}\right)\right\} .
\end{aligned}
$$

and the zero section

$$
E_{0}=\{(x, 0) \in E\} .
$$

We say that the cocycle $L$ is uniformly expanding if there exist $K>0$ and $\theta>1$ such that for every $(x, v) \in E$ we have

$$
\left|v_{i}\right| \geq K \theta^{i}|v|
$$

for every $i \geq 0$, where $L^{i}(x, v)=\left(x_{n}, v_{n}\right)$.

Proposition 5.2. The cocycle $L: E \rightarrow E$ is uniformly expanding if and only if

$$
\mathfrak{B}_{+}=E_{0} .
$$

Proof. Of course if $L: E \rightarrow E$ is uniformly expanding then (39) holds. To prove the reverse implication, note that (39) implies $\mathcal{S}=\mathfrak{B}=E_{0}$. By Theorem 2 in Sacker and Sell [35] (see also Section 7 there) we have that $L: E \rightarrow E$ is uniformly expanding.

One can also prove Proposition 5.2 applying Sacker and Sell's results in [36, Lemma 9 and Theorem 2]. We just refer to that because the proof of these results in [36] seems to be more elementary than the proof of Theorem 2 in [35]. 
5.2. Unstable invariant cones. Let $F \in \Omega_{n, p}$, Denote

$$
\mathfrak{B}_{+}(F)=\left\{v \in T_{F} \mathcal{B}_{\text {nor }}(U) \text { s.t. } \sup _{i \in \mathbb{N}}\left|D \mathcal{R}_{f}^{i} \cdot v_{i}\right|<\infty\right\} .
$$

Consider the topological vector bundle $E$ (see Proposition 4.13)

$$
E=\left\{(F,[v]) \text { s.t. } F \in \Omega_{n, p} \text { and }[v] \in T_{F} \mathcal{B}_{\text {nor }}(U) / E_{F}^{h}\right\} .
$$

with the continuous function (35) that restriced to each $T_{F} \mathcal{B}_{\text {nor }}(U) / E_{F}^{h}$ is the usual quotient norm. Recall that due Proposition 4.13 we have that

$$
\operatorname{dim} T_{F} \mathcal{B}_{\text {nor }}(U) / E_{F}^{h}=n \text {. }
$$

By Proposition 4.2 the linear transformation

$$
D \mathcal{R}_{F}: T_{F} \mathcal{B}_{\text {nor }}(U) \rightarrow T_{\mathcal{R} F} \mathcal{B}_{\text {nor }}(U)
$$

induces a bounded linear transformation

$$
L_{F}: T_{F} \mathcal{B}_{\text {nor }} / E_{F}^{h} \rightarrow T_{\mathcal{R} F} \mathcal{B}_{\text {nor }}(U) / E_{\mathcal{R} F}^{h} .
$$

Lemma 5.3. The map

$$
L(F, v)=\left(\mathcal{R} F, L_{F} \cdot v\right)
$$

is a vector bundle isomorphism in the vector bundle $E$, that is, it is a homeomorphism of $E$ onto itself that preserves the linear structure on the fibers.

Proof. Let $\pi: \Omega_{n, p} \times T \mathcal{B}_{n o r}(U) \rightarrow E$ be a natural projection

$$
(F, v) \mapsto\left(F,[v]_{F}\right),
$$

where $[v]_{F}$ represents the equivalent class of $v$ in $T \mathcal{B}_{n o r}(U) / E_{F}^{h}$. Of course

$$
\tilde{L}: \Omega_{n, p} \times T \mathcal{B}_{n o r}(U) \rightarrow E
$$

defined by $\tilde{L}(F, v)=\pi\left(\mathcal{R} F, D \mathcal{R}_{F} \cdot v\right)$ is continuous. Then by Proposition 4.2 the map $\tilde{L}$ descends to the topological quotient space $E$ as the continuous map $L$.

By Theorem 3 we have that the linear transformation (40) has dense image in $T_{\mathcal{R} F} \mathcal{B}_{\text {nor }}(U)$. This implies that for every $F$ the linear map $L_{F}$ is invertible. By Corollary 2.3 we have that $\mathcal{R}: \Omega_{n, p} \rightarrow \Omega_{n, p}$ is a homeomorphism. We conclude that $L$ is invertible. It remains to show that its inverse is continuous. Since

$$
E^{1}=\left\{\left(F,[v]_{F}\right): F \in \Omega_{n, p} \text { and }\left|[v]_{F}\right|=1\right\}
$$

is compact, $L$ is invertible, and the function

$$
\psi: E^{1} \rightarrow \mathbb{R}^{+}
$$

defined by $\psi\left(F,[v]_{F}\right)=\left|L\left(F,[v]_{F}\right)\right|$ is continuous, we have that

$$
C=\min _{\left(F,[v]_{F}\right) \in E^{1}} \psi\left(F,[v]_{F}\right)>0 .
$$

So $\operatorname{suppose} \lim _{k}\left(F_{k},\left[v_{k}\right]_{F_{k}}\right)=\left(F,[v]_{F}\right)$ and

$$
L^{-1}\left(F_{k},\left[v_{k}\right]_{F_{k}}\right)=\left(\mathcal{R}^{-1} F_{k},\left[w_{k}\right]_{\mathcal{R}^{-1} F_{k}}\right)
$$

Then $\lim _{k} \mathcal{R}^{-1} F_{k}=\mathcal{R}^{-1} F$ and by (41) we have $\sup _{k}\left|\left[w_{k}\right]_{\mathcal{R}^{-1} F_{k}}\right| \leq \hat{C}$ for some constant $\hat{C}$. Taking a subsequence we can assume that $\lim _{k}\left[w_{k}\right]_{\mathcal{R}^{-1} F_{k}}=[\tilde{w}]_{\mathcal{R}^{-1} F}$. Since $L$ is continuous

$$
\left(F,[v]_{F}\right)=\lim _{k} L\left(\mathcal{R}^{-1} F_{k},\left[w_{k}\right]_{\mathcal{R}^{-1} F_{k}}\right)=\left(F, L_{F}[\tilde{w}]_{\mathcal{R}^{-1} F}\right) .
$$


From the injectivity of $L$ we conclude that $[\tilde{w}]_{\mathcal{R}^{-1} F}=L_{F}^{-1}[v]_{F}$. So $L^{-1}$ is continuous.

Proposition 5.4. Suppose that for every $F \in \Omega_{n, p}$ we have

$$
\mathfrak{B}_{+}(F) \subset E_{F}^{h} \text {. }
$$

Then the cocycle $L$ is uniformly expanding, that is, there is $C>0$ and $\theta_{2}>1$ such that for every $v \in \mathcal{B}_{\text {nor }}(U)$ and $F \in \Omega_{n, p}$ we have

$$
d\left(D \mathcal{R}_{F}^{i} \cdot v, E_{\mathcal{R}^{i} F}^{h}\right) \geq C \theta_{2}^{i} d\left(v, E_{F}^{h}\right) .
$$

Proof. Indeed, suppose that $[v] \in T_{F} \mathcal{B}_{\text {nor }} / E_{F}^{h}$ satisfies

$$
\sup _{i}\left|L_{F}^{i} \cdot[v]\right|<\infty \text {. }
$$

By Propostion 5.2, it is enough to show that $[v]=0$, that is, $v \in E_{F}^{h}$. Firstly note that $D \mathcal{R}_{F}^{i} \cdot v=u_{i}+w_{i}$, where $\sup _{i}\left|u_{i}\right|=C<\infty$ and $w_{i} \in E_{\mathcal{R}^{i} F}^{h}$. Note that

$$
D \mathcal{R}_{\mathcal{R}^{i} F} \cdot\left(u_{i}+w_{i}\right)=u_{i+1}+w_{i+1},
$$

SO

So

in particular

$$
w_{i+1}=D \mathcal{R}_{\mathcal{R}^{i} F} \cdot u_{i}-u_{i+1}+D \mathcal{R}_{\mathcal{R}^{i} F} \cdot w_{i}
$$

$$
w_{i+j}=D \mathcal{R}_{\mathcal{R}^{i} F}^{j} \cdot u_{i}-u_{i+j}+D \mathcal{R}_{\mathcal{R}^{i} F}^{j} \cdot w_{i}
$$

$$
\left|w_{i+j}\right| \leq C\left(1+K \theta^{-j}\right)+K \theta^{-j}\left|w_{i}\right|,
$$

where $K$ and $\theta>1$ are as in Proposition 4.5. This implies that $\sup _{i}\left|w_{i}\right|<\infty$ and consequently

$$
\sup _{i}\left|D \mathcal{R}_{F}^{i} \cdot v\right|<\infty \text {. }
$$

By (37) we have that $v \in E_{F}^{h}$. So $L$ is uniformly expanding.

Let $C>0$ and $\theta>1$ be as in (43). Choose $j_{0}>0$ such that

$$
C \theta^{j_{0}}>1 \text {. }
$$

If $\epsilon>0$ is small enough we have that

$$
\tilde{\theta}=C e^{-\epsilon} \theta^{j_{0}}>1 \text {. }
$$

Denote

$$
\tilde{C}=\sup _{F \in \Omega_{n, p}}\left|D \mathcal{R}_{F}^{j_{0}}\right| .
$$

Define the cone $C_{F}^{u}(K)$ as the set of all $v \in T \mathcal{B}_{\text {nor }}(U)$ that can be written as $v=u+w$, where
A. $|u| \leq e^{\epsilon} d\left(v, E_{F}^{h}\right)$,
B. $w \in E_{F}^{h}$ and
C. $|w| \leq K|u|$.

Note that

$$
\bigcup_{K>0} C_{F}^{u}(K)=\left(T \mathcal{B}_{\text {nor }}(U) \backslash E_{F}^{h}\right) \cup\{0\} .
$$

Our goal is to show that if (43) holds then there is $K>0$ such that

$$
F \in \Omega_{n, p} \mapsto C_{F}^{u}(K)
$$

is a field of unstable $\mathcal{R}$-invariant cones on $\Omega_{n, p}$. 
Proposition 5.5. Assume that (43) holds. Then for $\epsilon>0$ small enough the following holds. Let $F \in \Omega_{n, p}$. If $v_{0}=u_{0}+w_{0}$, with

$$
0<\left|u_{0}\right| \leq e^{\epsilon} d\left(v_{0}, E_{F}^{h}\right) \text { and } w_{0} \in E_{F}^{h} .
$$

then for every $w_{1} \in E_{\mathcal{R}^{j 0} F}^{h}$ and $u_{1}$ satisfying $D \mathcal{R}_{F}^{j_{0}} \cdot v_{0}=u_{1}+w_{1}$ we have

$$
\frac{\left|w_{1}\right|}{\left|u_{1}\right|} \leq \tilde{C} \tilde{\theta}^{-1}+1+\tilde{\theta}^{-2} \frac{\left|w_{0}\right|}{\left|u_{0}\right|}
$$

Proof. Let $v_{1}=D \mathcal{R}_{F}^{j_{0}} \cdot v_{0}$. In particular

$$
\frac{\left|u_{1}\right|}{\left|u_{0}\right|} \geq e^{-\epsilon} \frac{d\left(D \mathcal{R}_{F}^{j_{0}} \cdot v_{0}, E_{\mathcal{R}^{j_{0}}}^{h}\right)}{d\left(v_{0}, E_{F}^{h}\right)} \geq C e^{-\epsilon} \theta^{j_{0}}=\tilde{\theta}>1 .
$$

Then

$$
D \mathcal{R}_{F}^{j_{0}} v_{0}=D \mathcal{R}_{F}^{j_{0}} u_{0}+D \mathcal{R}_{F}^{j_{0}} w_{0}=u_{1}+w_{1}
$$

So

and

$$
D \mathcal{R}_{F}^{j_{0}} \frac{u_{0}}{\left|u_{1}\right|}+D \mathcal{R}_{F}^{j_{0}} \frac{w_{0}}{\left|u_{1}\right|}=\frac{u_{1}}{\left|u_{1}\right|}+\frac{w_{1}}{\left|u_{1}\right|}
$$

$$
\begin{aligned}
\frac{\left|w_{1}\right|}{\left|u_{1}\right|} & \leq\left|D \mathcal{R}_{F}^{j_{0}}\right| \frac{\left|u_{0}\right|}{\left|u_{1}\right|}+1+\left|D \mathcal{R}_{F}^{j_{0}} \frac{w_{0}}{\left|u_{1}\right|}\right| \\
& \leq \tilde{C} \tilde{\theta}^{-1}+1+C \theta^{-j_{0}} \frac{\left|w_{0}\right|}{\left|u_{1}\right|} \\
& \leq \tilde{C} \tilde{\theta}^{-1}+1+\tilde{\theta}^{-1} C \theta^{-j_{0}} \frac{\left|w_{0}\right|}{\left|u_{0}\right|} \\
& \leq \tilde{C} \tilde{\theta}^{-1}+1+\tilde{\theta}^{-2} \frac{\left|w_{0}\right|}{\left|u_{0}\right|} .
\end{aligned}
$$

Corollary 5.6 (Invariant Cones). Assume that (43) holds. If $v_{0} \in C_{F}^{u}\left(K_{0}\right)$ then $v_{1}=D \mathcal{R}_{F}^{j_{0}} \cdot v_{0} \in C_{\mathcal{R} F}^{u}\left(K_{1}\right)$, where

$$
K_{1}=\tilde{C} \tilde{\theta}^{-1}+1+\tilde{\theta}^{-2} K_{0} .
$$

Proof. We can assume that $v_{0} \neq 0$. Since $v_{0} \in C_{F}^{u}\left(K_{0}\right)$ there exist $w_{0} \in E_{F}^{h}$ and $u_{0}$ such that $v_{0}=u_{0}+w_{0}$ and

$$
0<\left|u_{0}\right| \leq e^{\epsilon} d\left(v_{0}, E_{F}^{h}\right) \text { and }\left|w_{0}\right| \leq K_{0}\left|u_{0}\right| .
$$

Moreover there exist $w_{1} \in E_{\mathcal{R}^{j_{0} F}}^{h}$ and $u_{1}$ satisfying $v_{1}=u_{1}+w_{1}$, with

$$
\left|u_{1}\right| \leq e^{\epsilon} d\left(v_{1}, E_{\mathcal{R}^{j_{0} F}}^{h}\right) .
$$

By Proposition 5.5 we have that $\left|w_{1}\right| \leq K_{1}\left|u_{1}\right|$, so $v_{1} \in C_{\mathcal{R}^{j_{0} F}}^{u}\left(K_{1}\right)$.

To simplify the notation, we will replace the operator $\mathcal{R}$ by its iteration $\mathcal{R}^{j_{0}}$. The following two corollaries are an immediate consequence of Corollary 5.6.

Corollary 5.7 (Forward Invariant Cones). Assume that (43) holds. If

$$
K \geq \frac{\tilde{C} \tilde{\theta}^{-1}+1}{1-\tilde{\theta}^{-2}}
$$


then for every $F \in \Omega_{n, p}$

$$
D \mathcal{R}_{F} C_{F}^{u}(K) \subset C_{\mathcal{R} F}^{u}(K) .
$$

Corollary 5.8 (Absorbing Cones). Assume that (43) holds. For each

$$
K_{0}>\frac{\tilde{C} \tilde{\theta}^{-1}+1}{1-\tilde{\theta}^{-2}}
$$

the following holds: for every $K>0$ there exists $i$ such that for all $F \in \Omega_{n, p}$

$$
D \mathcal{R}_{F}^{i} C_{F}^{u}(K) \subset C_{\mathcal{R}^{i} F}^{u}\left(K_{0}\right) .
$$

Corollary 5.9 (Unstable Cones). Assume that (43) holds. For each $K_{0}>0$ there exists $C>0$ such that for all $F \in \Omega_{n, p}, v \in C_{F}^{u}\left(K_{0}\right)$ and $i \geq 0$

$$
\left|D \mathcal{R}_{F}^{i} v\right| \geq C \theta^{i}|v| \text {. }
$$

Proof. If $v \in C_{F}^{u}\left(K_{0}\right)$ then $v=u+w$, with $w \in E_{F}^{h}$,

$$
|u| \leq e^{\epsilon} d\left(v, E_{F}^{h}\right)
$$

and

So

$$
|w| \leq K_{0}|u|
$$

$$
|v| \leq|u|+|w| \leq\left(1+K_{0}\right) e^{\epsilon} d\left(v, E_{F}^{h}\right) .
$$

By (43) we have

$$
\left|D \mathcal{R}_{F}^{i} \cdot v\right| \geq d\left(D \mathcal{R}_{F}^{i} \cdot v, E_{\mathcal{R}^{i} F}^{h}\right) \geq C \theta^{i} d\left(v, E_{F}^{h}\right) \geq \frac{C}{\left(1+K_{0}\right) e^{\epsilon}} \theta^{i}|v| .
$$

Now fix

$$
K_{0}>\frac{\tilde{C} \tilde{\theta}^{-1}+1}{1-\tilde{\theta}^{-2}} .
$$

Choose $i>0$ such that

$$
\theta_{1}=\frac{C}{\left(1+K_{0}\right) e^{\epsilon}} \theta^{i}>1 .
$$

Replace (once again) the operator $\mathcal{R}$ by its iteration $\mathcal{R}^{i}$.

Corollary 5.10 (Unstable Invariant Cones near $\Omega_{n, p}$ ). Assume that (43) holds. For each

$$
K_{0}>\frac{\tilde{C} \tilde{\theta}^{-1}+1}{1-\tilde{\theta}^{-2}} .
$$

and $\hat{\theta} \in\left(1, \theta_{1}\right)$ there exists $\delta>0$ such that if

$$
\operatorname{dist}\left(F, G_{0}\right)<\delta
$$

for some $G_{0} \in \Omega_{n, p}$ then

$$
D \mathcal{R}_{F} C_{G_{0}}^{u}\left(K_{0}\right) \subset C_{\mathcal{R} G_{0}}^{u}\left(K_{0}\right)
$$

and

$$
\left|D \mathcal{R}_{F} \cdot v\right| \geq \hat{\theta}|v|
$$

for every $v \in C_{G_{0}}^{u}\left(K_{0}\right)$. 
Proof. Define $K_{1}=\tilde{C} \tilde{\theta}^{-1}+1+\tilde{\theta}^{-2} K_{0}$. Then

$$
\frac{\tilde{C} \tilde{\theta}^{-1}+1}{1-\tilde{\theta}^{-2}}<K_{1}<K_{0}
$$

Choose $\gamma \in(0, \epsilon)$ small enough such that

$$
K_{1} e^{\gamma}<K_{0} .
$$

Let $v \in C_{G_{0}}^{u}\left(K_{0}\right)$. Then there exist $w_{0} \in E_{G_{0}}^{h}$ and $u_{0}$ such that $v=u_{0}+w_{0}$ and

$$
\left|u_{0}\right| \leq e^{\epsilon} d\left(v_{0}, E_{G_{0}}^{h}\right) \text { and }\left|w_{0}\right| \leq K_{0}\left|u_{0}\right| .
$$

Moreover there exist $w_{1} \in E_{\mathcal{R} G_{0}}^{h}$ and $u_{1}$ satisfying

$$
\left|u_{1}\right| \leq e^{\gamma / 3} d\left(D \mathcal{R}_{G_{0}} \cdot v, E_{\mathcal{R} G_{0}}^{h}\right)
$$

and $D \mathcal{R}_{G_{0}} \cdot v=u_{1}+w_{1}$. By Proposition 5.5

$$
\left|w_{1}\right| \leq K_{1}\left|u_{1}\right| .
$$

Then

$$
D \mathcal{R}_{F} \cdot v=u_{1}+\left(D \mathcal{R}_{G_{0}}-D \mathcal{R}_{F}\right) \cdot v+w_{1} .
$$

Note that

$$
\begin{aligned}
& d\left(\left(D \mathcal{R}_{G_{0}}-D \mathcal{R}_{F}\right) \cdot v, E_{\mathcal{R} G_{0}}^{h}\right) \\
\leq & \left|\left(D \mathcal{R}_{G_{0}}-D \mathcal{R}_{F}\right) \cdot v\right| \\
\leq & \left|D \mathcal{R}_{G_{0}}-D \mathcal{R}_{F}\right| e^{\epsilon}\left(1+K_{0}\right) d\left(v, E_{G_{0}}^{h}\right) \\
\leq & \left|D \mathcal{R}_{G_{0}}-D \mathcal{R}_{F}\right| \frac{e^{\epsilon}\left(1+K_{0}\right)}{\theta} d\left(D \mathcal{R}_{G_{0}} \cdot v, E_{\mathcal{R} G_{0}}^{h}\right)
\end{aligned}
$$

Let $\delta_{1}>0$ be such that $\left|F-G_{0}\right|<\delta_{1}$ implies

$$
\begin{gathered}
1-\left|D \mathcal{R}_{G_{0}}-D \mathcal{R}_{F}\right| \frac{e^{\epsilon}\left(1+K_{0}\right)}{\theta} \geq e^{-\gamma / 3}, \\
e^{\gamma / 3}+\left|D \mathcal{R}_{G_{0}}-D \mathcal{R}_{F}\right| \frac{e^{\epsilon}\left(1+K_{0}\right)}{\theta} \leq e^{2 \gamma / 3},
\end{gathered}
$$

and

$$
\tilde{\theta}=\theta_{1}-\left|D \mathcal{R}_{G_{0}}-D \mathcal{R}_{F}\right|>\hat{\theta}>1 .
$$

Then

$$
\begin{aligned}
& d\left(D \mathcal{R}_{F} \cdot v, E_{\mathcal{R} G_{0}}^{h}\right) \\
\geq & d\left(D \mathcal{R}_{G_{0}} \cdot v, E_{\mathcal{R} G_{0}}^{h}\right)-d\left(\left(D \mathcal{R}_{G_{0}}-D \mathcal{R}_{F}\right) \cdot v, E_{\mathcal{R} G_{0}}^{h}\right) \\
\geq & e^{-\gamma / 3} d\left(D \mathcal{R}_{G_{0}} \cdot v, E_{\mathcal{R} G_{0}}^{h}\right)
\end{aligned}
$$

so

$$
\begin{aligned}
\left|u_{1}+\left(D \mathcal{R}_{G_{0}}-D \mathcal{R}_{F}\right) \cdot v\right| & \leq e^{\gamma / 3} d\left(D \mathcal{R}_{G_{0}} \cdot v, E_{\mathcal{R} G_{0}}^{h}\right)+\left|\left(D \mathcal{R}_{G_{0}}-D \mathcal{R}_{F}\right) \cdot v\right| \\
& \leq e^{2 \gamma / 3} d\left(D \mathcal{R}_{G_{0}} \cdot v, E_{\mathcal{R} G_{0}}^{h}\right) \\
& \leq e^{\gamma} d\left(D \mathcal{R}_{F} \cdot v, E_{\mathcal{R} G_{0}}^{h}\right) \\
& \leq e^{\epsilon} d\left(D \mathcal{R}_{F} \cdot v, E_{\mathcal{R} G_{0}}^{h}\right),
\end{aligned}
$$


and moreover

$$
\begin{aligned}
\left|u_{1}+\left(D \mathcal{R}_{G_{0}}-D \mathcal{R}_{F}\right) \cdot v\right| & \geq\left|u_{1}\right|-\left|\left(D \mathcal{R}_{G_{0}}-D \mathcal{R}_{F}\right) \cdot v\right| \\
& \geq d\left(D \mathcal{R}_{G_{0}} \cdot v, E_{\mathcal{R} G_{0}}^{h}\right)-\left|\left(D \mathcal{R}_{G_{0}}-D \mathcal{R}_{F}\right) \cdot v\right| \\
& \geq e^{-\gamma / 3} d\left(D \mathcal{R}_{G_{0}} \cdot v, E_{\mathcal{R}_{0}}^{h}\right) .
\end{aligned}
$$

Finally

$$
\begin{aligned}
\left|w_{1}\right| & \leq K_{1}\left|u_{1}\right| \\
& \leq K_{1} e^{\gamma / 3} d\left(D \mathcal{R}_{G_{0}} \cdot v, E_{\mathcal{R} G_{0}}^{h}\right) \\
& \leq K_{1} e^{2 \gamma / 3}\left|u_{1}+\left(D \mathcal{R}_{G_{0}}-D \mathcal{R}_{F}\right) \cdot v\right| . \\
& \leq K_{0}\left|u_{1}+\left(D \mathcal{R}_{G_{0}}-D \mathcal{R}_{F}\right) \cdot v\right| .
\end{aligned}
$$

So (52), (55) and (57) implies that $D \mathcal{R}_{F} \cdot v \in C_{\mathcal{R} G_{0}}^{u}\left(K_{0}\right)$. Furthermore

$$
\begin{aligned}
\left|D \mathcal{R}_{F} \cdot v\right| & \geq\left|D \mathcal{R}_{G} \cdot v\right|-\left|\left(D \mathcal{R}_{G_{0}}-D \mathcal{R}_{F}\right) \cdot v\right| \\
& \geq \theta_{1}|v|-\left|D \mathcal{R}_{G_{0}}-D \mathcal{R}_{F}\right||v| \\
& \geq \hat{\theta}|v| .
\end{aligned}
$$

Proof of Proposition 5.1. Let $K_{0}$ be as in Corollary 5.10. We claim that every cone $C_{F}^{u}\left(K_{0}\right)$, with $F \in \Omega_{n, p}$, contains a subspace $S_{F}$ of dimension $n$. Indeed, since $E_{G}^{h}, G \in \Omega_{n, p}$, has finite codimension $n$ there is a subspace $E_{G} \subset T \mathcal{B}_{\text {nor }}(U)$ of dimension $n$ such that

$$
E_{G} \oplus E_{G}^{h}=T \mathcal{B}_{\text {nor }}(U) .
$$

Since $\Omega_{n, p}$ is a Cantor set and $G \mapsto E_{G}^{h}$ is a continuous distribution, it is easy to see that we can find a finite covering $\left\{O_{j}\right\}_{j}$ by compact subsets of $\Omega_{n, p}$ and subspaces $E_{j}$ with dimension $n$ such that

$$
E_{j} \oplus E_{G}^{h}=T \mathcal{B}_{\text {nor }}(U)
$$

for every $G \in O_{j}$. By (44), Proposition 5.5 and Corollary 5.8 there exists $i_{0}$ such that

$$
D \mathcal{R}_{G}^{i_{0}} E_{j} \subset C_{\mathcal{R}^{i_{0}} G}^{u}\left(K_{0}\right) .
$$

for every $G \in O_{j}$. Moreover $\mathcal{R}$ is invertible on $\Omega_{n, p}$, so we can choose $G$ such that $\mathcal{R}^{i_{0}} G=F$. Since $D \mathcal{R}_{G}$ is injective for every $G \in \Omega_{n . p}$ we conclude that $S_{F}=D \mathcal{R}_{G}^{i_{0}} E_{j}$ is a subspace of dimension $n$ in $C_{F}^{u}\left(K_{0}\right)$. This concludes the proof of the claim. Note that

$$
S_{F} \oplus E_{F}^{h}=T \mathcal{B}_{\text {nor }}(U) .
$$

Let $G \in \Omega_{n, p}$. Since $\mathcal{R}$ is invertible on $\Omega_{n, p}$ there is a unique sequence $G_{i} \in \Omega_{n, p}$ such that $\mathcal{R} G_{i+1}=G_{i}$ and $G_{0}=G$. Let $S_{i}^{\prime}$ be an arbritrary subspace of dimension $n$ contained in $C_{G_{i}}^{u}\left(K_{0}\right)$, Then $D \mathcal{R}_{G_{i}}^{i}\left(S_{i}^{\prime}\right)$ is a subspace of dimension $n$ contained in $C_{G}^{u}\left(K_{0}\right)$. Since

$$
C_{G}^{u}\left(K_{0}\right) \cap E_{G}^{h}=\{0\}
$$

we have that there is a linear function

$$
\mathcal{H}_{i}: S_{G} \rightarrow E_{G}^{h}
$$


such that $\left\{v+\mathcal{H}_{i}(v), v \in S_{G}\right\}=D \mathcal{R}_{G_{i}}^{i}\left(S_{i}^{\prime}\right)$. Since $v+\mathcal{H}_{i}(v) \in C_{G}^{u}\left(K_{0}\right)$ we have $v+\mathcal{H}_{i}(v)=u_{1}+w_{1}$, with $w_{1} \in E_{G}^{h}$ and

$$
\left|u_{1}\right| \leq e^{\epsilon} d\left(v+\mathcal{H}_{i}(v), E_{G}^{h}\right)=e^{\epsilon} d\left(v, E_{G}^{h}\right)<e^{\epsilon}|v| .
$$

and $\left|w_{1}\right| \leq K_{0}\left|u_{1}\right|$. In particular

$$
\left|\mathcal{H}_{i}(v)\right|=\left|u_{1}+w_{1}-v\right| \leq\left(1+e^{\epsilon}\left(1+K_{0}\right)\right)|v|,
$$

So the family of functions $\mathcal{H}_{i}$ is an equicontinuous family of functions. By the strong compactness of the operator $\mathcal{R}$ and Arzela-Ascoli Theorem there exists a subsequence that converges to a bounded linear function $\mathcal{H}^{G}: S_{G} \rightarrow E_{G}^{h}$ satisfying

$$
\left\{v+\mathcal{H}^{G}(v), v \in S_{G}\right\} \subset \bigcap_{i=0}^{\infty} \overline{D \mathcal{R}_{G_{i}}^{i} C_{G_{i}}^{u}\left(K_{0}\right)} \subset C_{G}^{u}\left(K_{0}\right) .
$$

Due (59) and the contraction in the horizontal directions we have that there is only one possible accumulation point $\mathcal{H}^{G}$ for sequences as $\left(\mathcal{H}_{i}\right)_{i}$. Let

$$
E_{G}^{u}=\left\{v+\mathcal{H}^{G}(v), v \in S_{G}\right\} .
$$

Then we can easily conclude that $D \mathcal{R}_{G}\left(E_{G}^{u}\right)=E_{\mathcal{R} G}^{u}$. Then $G \mapsto E_{G}^{u}$ is the unstable direction of $\mathcal{R}$.

\section{INDUCED EXPANDING MAPS.}

Let $F \in \Omega_{n, p}$. We are going to define a real induced map $G_{\mathbb{R}}: D \rightarrow \mathbb{R}$ for $F$ whose domain $D$ is the union of intervals $R_{-i}^{k}, k \geq 0$ and $0<i<n_{r}^{k}, r \in C(F)$, satisfying

$$
R_{-i}^{k} \subset \bigcup_{q \in C(F)} Q_{0}^{k-1} .
$$

If $R_{-i}^{k} \subset Q_{0}^{k-1}$ and $s \in C(F)$ is the sucessor of $q$ at the $k-1$ level we define $G_{\mathbb{R}}(x)=F^{n_{s}^{k-1}}(x)$ for every $x \in R_{-i}^{k}$. Note that

$$
G_{\mathbb{R}}: R_{-i}^{k} \rightarrow R_{-i+n_{s}^{k-1}}^{k}
$$

is a diffeomorphism. Due the real bounds there exists $\epsilon_{0}$ such that

$$
0<\epsilon_{0}<\inf _{r \in C(F), k} \frac{2 \operatorname{dist}\left(P(F), \partial R_{0}^{k}\right)}{\left|R_{0}^{k}\right|} .
$$

We will now define a complex-analytic extension $G$ of $G_{\mathbb{R}}$ that is a conformal iterated dynamical system. Suppose that $R_{-i}^{k} \subset Q_{0}^{k-1}$. Let $\mathscr{R}_{0}^{k}$ be the ball $B\left(r, d_{r}^{k}\right)$, where

$$
d_{r}^{k}=\left(1-\frac{\epsilon_{0}}{16}\right) \frac{\left|R_{0}^{k}\right|}{2} .
$$

Since $F$ belongs to the Epstein class, there exists a simply connected domain $\mathscr{R}_{-i}^{k}$ such that $\mathscr{R}_{-i}^{k} \cap \mathbb{R} \subset R_{-i}^{k}$ and $\mathscr{R}_{-i}^{k}$ is contained in the ball whose diameter is $R_{-i}^{k} \cap \mathbb{R}$ and moreover

$$
F^{i}: \mathscr{R}_{-i}^{k} \rightarrow \mathscr{R}_{0}^{k}
$$

is univalent. Due the real bounds, we can reduce $\epsilon_{0}$ if necessary in such way that

$$
\mathscr{R}_{-i}^{k} \subset \mathcal{Q}_{0}^{k-1}
$$




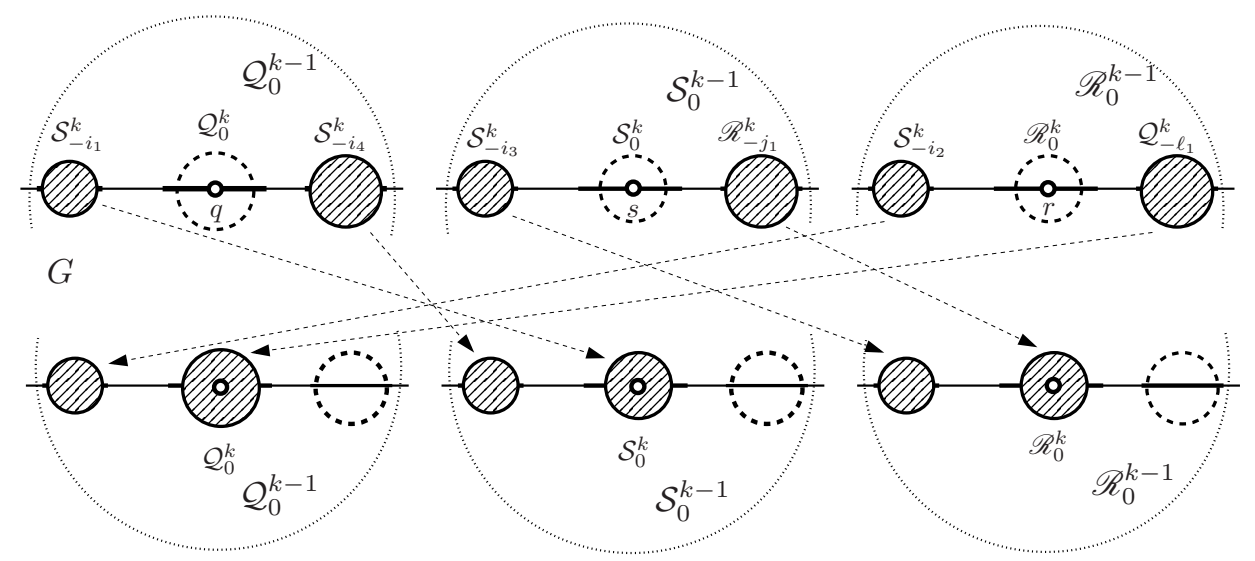

Figure 2. How $G$ acts on the domains $\mathscr{R}_{-i}^{k} \subset \cup_{q \in C(F)} \mathcal{Q}_{0}^{k-1}$ if the combinatorics of the $k$-th renormalization is the same as the combinatorics of the renormalization $R(F)$ in Fig. 1. At level $k-1$ we have that $s$ is the sucessor of $q, r$ is the sucessor of $s$ and $q$ is the sucessor of $r$. At level $k$ we have that $r$ is the sucessor of $q, s$ is the sucessor of $r$ and $q$ is the sucessor of $s$. Moreover $\ell_{1}, j_{1}>0$ and $0<i_{1}<i_{2}<i_{3}<i_{4}$.

for every $R_{-i}^{k} \subset Q_{0}^{k-1}$ and

$$
\inf _{k} \inf _{\substack{\{q, r\} \subset C(F) \\ R_{-i}^{k} \subset Q_{0}^{k-1}}} \operatorname{dist}\left(\mathscr{R}_{-i}^{k}, \partial \mathcal{Q}_{0}^{k-1}\right)>0
$$

We define $G$ on

$$
\mathscr{D}=\bigcup_{k} \bigcup_{r, q \in C(F)} \bigcup_{R_{-i}^{k} \subset Q_{0}^{k-1}} \mathscr{R}_{-i}^{k}
$$

as $G(z)=F^{n_{s}^{k-1}}(z)$ for every $z \in \mathscr{R}_{-i}^{k}$, where $R_{-i}^{k} \subset Q_{0}^{k-1}$ and $s \in C(F)$ is the sucessor of $q$ at level $k-1$. Note that $P(F) \backslash C(F) \subset \mathscr{D}$ and

$$
G(P(F) \backslash C(F))=P(F) .
$$

Lemma 6.1 (Markovian property of the induced map). Let $r_{j} \in C(F), m_{i} \in \mathbb{N}$ and $0 \leq i_{j}<n_{r_{j}}^{m_{i}}, j \leq \ell$ be such that

A. either we have that $m_{j+1}=m_{j}, i_{j+1}<i_{j}, r_{j+1}=r_{j}=r$ for some $r \in C(F)$ and moreover

$$
R_{-i_{j}}^{m_{j}}, R_{-i_{j+1}}^{m_{j}} \subset \bigcup_{q \in C(F)} Q_{0}^{m_{j}-1}
$$

and

$$
R_{-i}^{m_{j}} \notin \bigcup_{q \in C(F)} Q_{0}^{m_{j}-1}
$$

for every $i$ satisfying $i_{j+1}<i<i_{j}$. In particular

$$
G_{\mathbb{R}}: R_{-i_{j}}^{m_{j}} \mapsto R_{-i_{j+1}}^{m_{j+1}} .
$$

is a diffeomorphism. 
B. or

$$
\begin{gathered}
m_{j+1}>m_{j}, \\
\quad R_{-i_{j}}^{m_{j}} \subset \bigcup_{q \in C(F)} Q_{0}^{m_{j}-1},
\end{gathered}
$$

and

$$
R_{-i}^{m_{j}} \notin \bigcup_{q \in C(F)} Q_{0}^{m_{j}-1}
$$

for every $i$ satisfying $0<i<i_{j}$. Here $r=r_{j}$. In particular

$$
G: R_{-i_{j}}^{m_{j}} \mapsto R_{0}^{m_{j}} .
$$

is a diffeomorphism. Moreover $i_{j+1}>0$ and

$$
S_{-i_{j+1}}^{m_{j+1}} \subset R_{0}^{m_{j+1}-1}
$$

where $s=r_{j+1}$. In particular

$$
G_{\mathbb{R}}: R_{-i_{j}}^{m_{j}} \mapsto R_{0}^{m_{j}} .
$$

is a diffeomorphism and

$$
S_{-i_{j+1}}^{m_{j+1}} \subset R_{0}^{m_{j}}=G_{\mathbb{R}}\left(R_{-i_{j}}^{m_{j}}\right) .
$$

Then there exists a unique interval $W$ such that

$$
G_{\mathbb{R}}^{\ell}: W \rightarrow R_{-i_{\ell}}^{m_{\ell}}
$$

is a diffeomorphism and $W$ is the set of points $z$ such that for every $j \leq \ell$ we have $G_{\mathbb{R}}^{j}(z) \in Q_{-i_{j}}^{m_{j}}$, where $q=r_{j}$. Moreover $W=R_{-i}^{m_{\ell}}$, for some $i$, where $r=r_{\ell}$.

Proof. If $\ell=0$, there is nothing to prove, since $W=R_{-i_{0}}^{m_{0}}$, with $r=r_{0}$. Suppose by induction on $\ell$ that Lemma 6.1 holds for $\ell$. Let $r_{j} \in C(F), m_{i} \in \mathbb{N}$ and $0<i_{j}<n_{r_{j}}^{m_{i}}, j \leq \ell+1$ be as in the statement of the lemma. By the induction assumption there exists $b$ such that

$$
G_{\mathbb{R}}^{\ell}: R_{-b}^{m_{\ell+1}} \rightarrow R_{-i_{\ell+1}}^{m_{\ell+1}}
$$

is a diffeomorfism and for every $j \leq \ell$ we have $G_{\mathbb{R}}^{j} R_{-b}^{m_{\ell+1}} \subset Q_{-i_{j+1}}^{m_{j+1}}$, where $q=r_{j+1}$. In particular $R_{-b}^{m_{\ell+1}} \subset S_{-i_{1}}^{m_{1}}$, with $s=r_{1}$. There are two cases. If $m_{0}=m_{1}$ then $S_{-i_{0}}^{m_{0}}=S_{-i_{0}}^{m_{1}}, i_{0}>i_{1}$, and

$$
G_{\mathbb{R}}: S_{-i_{0}}^{m_{1}} \rightarrow S_{-i_{1}}^{m_{1}}
$$

is a diffeomorphism, and $W=R_{-\left(b+i_{0}-i_{1}\right)}^{m_{\ell}}$ is the unique interval $W \subset S_{-i_{0}}^{m_{1}}$ such that $G_{\mathbb{R}}(W)=R_{-b}^{m_{\ell}}$. If $m_{1}>m_{0}$ then $S_{-i_{1}}^{m_{1}} \subset Q_{0}^{m_{0}}$, with $q=m_{0}$, and

$$
G: Q_{-i_{0}}^{m_{0}} \rightarrow Q_{0}^{m_{0}}
$$

is a diffeomorphism. Then $W=R_{-\left(b+i_{0}\right)}^{m_{\ell}}$ is the unique interval $W \subset Q_{-i_{0}}^{m_{0}}$ such that $G(W)=R_{-b}^{m_{\ell}}$.

The next proposition says that the postcritical set $P(F)$ of $F$ is the maximal invariant set of the induced map $G$.

Proposition 6.2. Given $(z, j) \in \mathscr{D}$ we have that $(z, j)$ belongs to $\mathscr{D} \backslash P(F)$ if and only if there exists $k_{0} \geq 0$ such that $G^{k}(z, j) \in \mathscr{D}$ for every $k<k_{0}$ and $G^{k_{0}}(z, j) \notin \mathscr{D}$. 


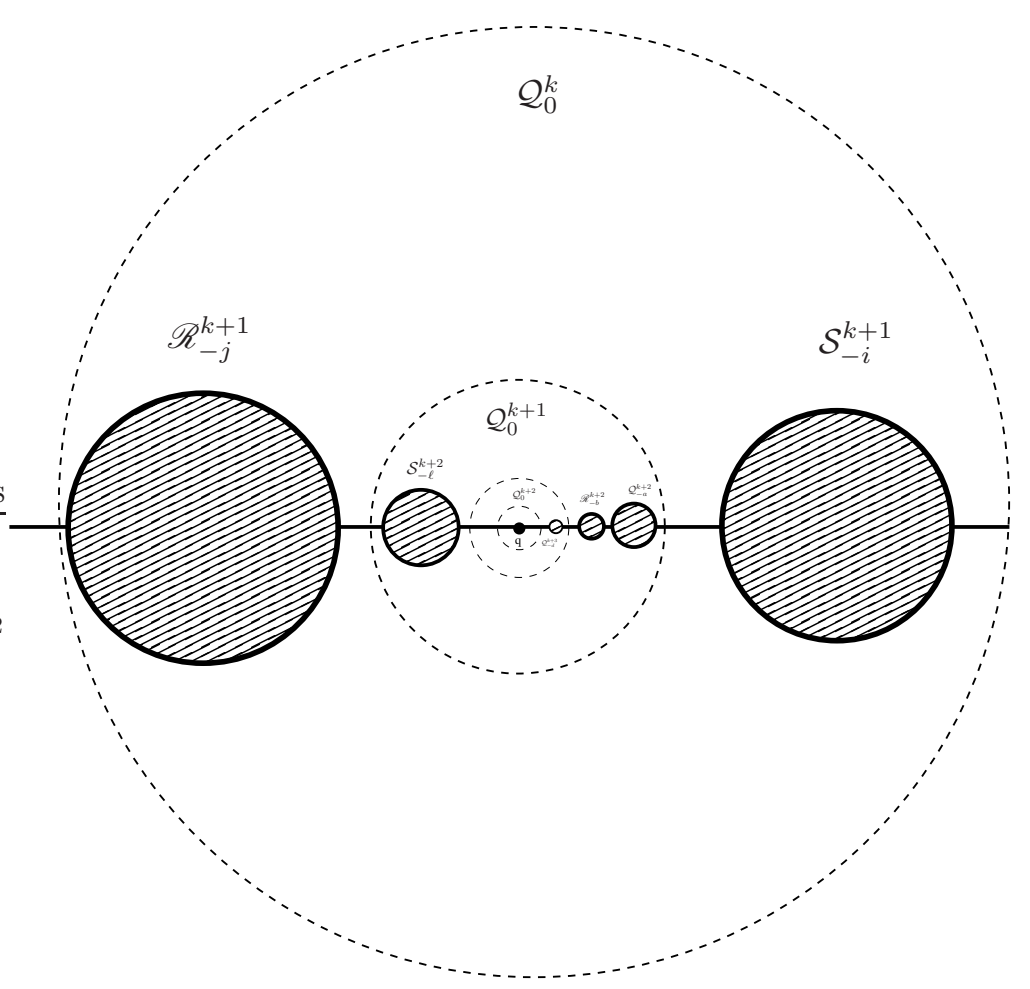

Figure 3 . The domain $\mathscr{D}$ of $G$ close to a critical point $q$ (the point in the center). It contains infinitely many topological disks accumulating on the critical point $q$.

Proof. Let $r_{j} \in C(F), m_{i} \in \mathbb{N}$ and $0<i_{j}<n_{r_{j}}^{m_{i}}$ such that either

$$
m_{j+1}>m_{j}
$$

and

$$
R_{-i_{j+1}}^{m_{j+1}} \subset Q_{0}^{m_{j}}
$$

where $r=r_{j+1}$ and $q=r_{j}$, or $m_{j+1}=m_{j}$ and $i_{j+1}=i_{j}-1$. We claim that there exists a unique $z \in \mathbb{C}_{n}$ such that

$$
G^{j}(z) \in \mathscr{R}_{-i_{j}}^{m_{j}}
$$

for every $j \geq 0$. Indeed, for each $\ell$, let $D_{\ell}$ be the set of points such that (62) holds for every $j \leq \ell$. Of course $D_{\ell+1} \subset D_{\ell}$. If $m_{\ell+1}=m_{\ell}$ then $D_{\ell+1}=D_{\ell}$. If $m_{\ell+1}>m_{\ell}$ then

with $q=r_{\ell}$ and

$$
G^{\ell+1}: D_{\ell} \rightarrow \mathcal{Q}_{0}^{m_{\ell}}
$$

$$
G^{\ell+1}: D_{\ell+1} \rightarrow \mathcal{R}_{-i_{\ell+1}}^{m_{\ell+1}},
$$

with $r=r_{\ell+1}$, are univalent (and onto). By the definition of $G$

so

$$
M=\inf _{m} \inf _{\mathcal{R}_{-i}^{m+1} \subset \mathcal{Q}_{0}^{m}} \bmod \left(\mathcal{Q}_{0}^{m} \backslash \mathcal{R}_{-i}^{m+1}\right)>0,
$$

$$
\bmod \left(D_{\ell} \backslash D_{\ell+1}\right)=\bmod \left(\mathcal{Q}_{0}^{m_{\ell}} \backslash \mathcal{R}_{-i_{\ell+1}}^{m_{\ell+1}}\right) \geq M
$$


in particular, since there exists infinitely many $\ell$ such that $m_{\ell+1}>m_{\ell}$ we have that

$$
\lim _{\ell} \operatorname{diam}\left(D_{\ell}\right)=0
$$

and

$$
\cap_{\ell} D_{\ell}=\left\{z_{0}\right\}
$$

for some $z_{0} \in \mathscr{D}$. This completes the proof of the claim. Note that since $D_{\ell}$ are symmetric with respect to the $\mathbb{R}$ we have that $z_{0} \in \mathbb{R}$ and by (62)

$$
G^{j}\left(z_{0}\right) \in \mathscr{R}_{-i_{j}}^{m_{j}} \cap \mathbb{R} \subset R_{-i_{j}}^{m_{j}},
$$

where $r=r_{j}$, for every $j$. By Lemma 6.1 there exists $b_{j}$ such that

$$
z_{0} \in R_{-b_{j}}^{m_{j}}
$$

where $r=r_{j}$, for every $j \geq 0$. In particular $z_{0} \in P(F)$.

\section{INDUCED PROBLEM.}

Let $a_{i}$ be as in (15). Define the function

$$
V: \mathscr{D} \rightarrow \mathbb{C}
$$

as

$$
V(z)=a_{i}(z)=\left.\frac{\partial}{\partial t}\left(F_{t}\right)^{n_{s}^{k-1}}(z)\right|_{t=0}
$$

for every $z \in \mathscr{R}_{-i}^{k} \subset \mathscr{D}$, provided $R_{-i}^{k} \subset Q_{0}^{k-1}$ and $s$ is the sucessor of $q$ at the $k-1$ level. Here $F_{t}=F+t v$.

Lemma 7.1. Suppose that $\alpha$ is a continuous vector field in a neighbourhood of $P(F)$, such that

A1. $\alpha(c)=0$ for every $c \in C(F)$, and

A2. We have

$$
V(z)=\alpha \circ G(z)-D G(z) \cdot \alpha(z)
$$

for every $z \in P(F) \backslash C(F)$.

Let $x \in P(F)$ and $\ell>0$ such that $G^{j}(x) \notin C(F)$ for every $0 \leq j<\ell$. Then for each $j<\ell$ there is $r, q \in C(F)$, with $b_{j}>0$ such that $G^{j}(x) \in R_{-b_{j}}^{k_{j}} \subset Q_{0}^{k_{j}-1}$. Define $i_{j}=n_{s}^{k_{j}-1}$, where $s \in C(F)$ is the sucessor of $q$ at level $k-1$. Note that the critical points $q, s, r$ may depend on $x$ and $j$. Let

$$
m=\sum_{j=0}^{\ell-1} i_{j} .
$$

Then

B1. For every $z \in \mathbb{C}$ close enough to $x$ we have that $G^{\ell}(z)$ is well defined and $G^{\ell}(z)=F^{m}(z)$.

B2. For every $z \in P(F)$ close enough to $x$ we have that $\alpha\left(G^{\ell}(z)\right)$ is well defined and

$$
\alpha(z)=\frac{\alpha\left(G^{\ell}(z)\right)}{D G^{\ell}(z)}-\sum_{a=1}^{m} \frac{v\left(F^{a-1}(z)\right)}{D F^{a}(z)} .
$$


B3. In particular for every $z \in P(F)$ close enough to $x$ such that $G^{\ell}(x) \in C(F)$ we have

$$
\alpha(x)=-\sum_{a=1}^{m} \frac{v\left(F^{a-1}(x)\right)}{D F^{a}(x)} .
$$

Proof. We let to the reader to show that $G^{\ell}(z)=F^{m}(z)$ for $z$ close enough to $x$. By (64) we get

$$
\alpha(x)=\frac{\alpha\left(G^{\ell}(z)\right)}{D G^{\ell}(z)}-\sum_{j=1}^{\ell} \frac{V\left(G^{j-1}(x)\right)}{D G^{j}(z)} .
$$

So

$$
\begin{gathered}
\sum_{j=1}^{\ell} \frac{V\left(G^{j-1}(x)\right)}{D G^{j}(z)}=\sum_{j=1}^{\ell} \frac{\left(\partial_{t}\left(F_{t}\right)^{i_{j}}\right)\left(F^{i_{0}+i_{1}+\cdots+i_{j-1}}(z)\right)}{D F^{i_{0}+i_{1}+\cdots+i_{j}}(z)} \\
=\sum_{j=1}^{\ell} \sum_{b=0}^{i_{j}-1} \frac{D F^{i_{j}-b-1}\left(F^{b+1}\left(F^{i_{0}+i_{1}+\cdots+i_{j-1}}(z)\right)\right) v\left(F^{b}\left(F^{i_{0}+i_{1}+\cdots+i_{j-1}}(z)\right)\right)}{D F^{i_{0}+i_{1}+\cdots+i_{j}}(z)} \\
=\sum_{j=1}^{\ell} \sum_{b=0}^{i_{j}-1} \frac{D F^{i_{j}-b-1}\left(F^{b+1}\left(F^{i_{0}+i_{1}+\cdots+i_{j-1}}(z)\right)\right) v\left(F^{i_{0}+i_{1}+\cdots+i_{j-1}+b}(z)\right)}{D F^{i_{j}-b-1}\left(F^{b+1}\left(F^{i_{0}+i_{1}+\cdots+i_{j-1}}(z)\right)\right) D F^{i_{0}+i_{1}+\cdots+i_{j-1}+b+1}(z)} \\
=\sum_{j=1}^{\ell} \sum_{b=0}^{i_{j}-1} \frac{v\left(F^{i_{0}+i_{1}+\cdots+i_{j-1}+b}(z)\right)}{D F^{i_{0}+i_{1}+\cdots+i_{j-1}+b+1}(z)}=\sum_{a=1}^{m} \frac{v\left(F^{a-1}(z)\right)}{D F^{a}(z)} .
\end{gathered}
$$

Proposition 7.2. Suppose that $\alpha$ is a continuous vector field in a neighbourhood of $P(F)$, such that $\alpha(r)=0$ for every $r \in C(F)$ and

$$
V(z)=\alpha \circ G(z)-D G(z) \cdot \alpha(z) .
$$

for every $z \in P(F) \backslash C(F)$. Then

$$
v(z)=\alpha \circ F(z)-D F(z) \cdot \alpha(z)
$$

for every $z \in P(F)$.

Proof. For each $R_{-m}^{k}$, with $r \in C(F)$ and $0<m<n_{r}^{k}$, there exists a unique $r_{-m}^{k} \in R_{-m}^{k}$ such that

$$
F^{m}\left(r_{-m}^{k}\right)=r
$$

The set

$$
\Gamma=\left\{r_{-m}^{k}\right\}_{r \in C(F), 0<m<n_{r}^{k}} \subset P(F)
$$

is dense on $P(F)$ and

$$
F(\Gamma)=\Gamma \cup C(F) .
$$

We claim that (66) holds for every $z \in \Gamma$. Indeed, given $r_{-m}^{k} \in \Gamma$, there exists $\ell>0$ such that $G^{\ell}\left(r_{-m}^{k}\right)=0$. Moreover by Lemma 7.1 we have that $G^{\ell}=F^{m}$ in a neighbourhood of $r_{-m}^{k}$ and

$$
\alpha\left(r_{-m}^{k}\right)=-\sum_{a=1}^{m} \frac{v\left(F^{a-1}\left(r_{-m}^{k}\right)\right)}{D F^{a}\left(r_{-m}^{k}\right)} .
$$


Suppose that $m=1$. Then $\ell=1, G=F$ in a neighbourhood of $r_{-1}^{k}, V\left(r_{-1}^{k}\right)=$ $v\left(r_{-1}^{k}\right)$ and $G\left(r_{-1}^{k}\right) \in C(F)$. By (65) it follows that

$$
v\left(r_{-1}^{k}\right)=\alpha \circ G\left(r_{-1}^{k}\right)-D G\left(r_{-1}^{k}\right) \cdot \alpha\left(r_{-1}^{k}\right)=\alpha \circ F\left(r_{-1}^{k}\right)-D F\left(r_{-1}^{k}\right) \cdot \alpha\left(r_{-1}^{k}\right) .
$$

If $m>1$ then $F\left(r_{-m}^{k}\right)=r_{-(m-1)}^{k} \in \Gamma$, so by (67) we have

$$
\begin{gathered}
\alpha\left(F\left(r_{-m}^{k}\right)\right)-D F\left(r_{-m}^{k}\right) \alpha\left(r_{-m}^{k}\right) \\
=-\sum_{a=1}^{m-1} \frac{v\left(F^{a-1}\left(r_{-(m-1)}^{k}\right)\right)}{D F^{a}\left(r_{-(m-1)}^{k}\right)}+D F\left(r_{-m}^{k}\right) \sum_{b=1}^{m} \frac{v\left(F^{b-1}\left(r_{-m}^{k}\right)\right)}{D F^{b}\left(r_{-m}^{k}\right)} \\
=-\sum_{a=1}^{m-1} \frac{v\left(F^{a-1}\left(r_{-(m-1)}^{k}\right)\right)}{D F^{a}\left(r_{-(m-1)}^{k}\right)}+\sum_{b=1}^{m} \frac{v\left(F^{b-1}\left(r_{-m}^{k}\right)\right)}{D F^{b-1}\left(r_{-(m-1)}^{k}\right)} \\
=-\sum_{a=1}^{m-1} \frac{v\left(F^{a-1}\left(r_{-(m-1)}^{k}\right)\right)}{D F^{a}\left(r_{-(m-1)}^{k}\right)}+v\left(r_{-m}^{k}\right)+\sum_{b=2}^{m} \frac{v\left(F^{b-2}\left(r_{-(m-1)}^{k}\right)\right)}{D F^{b-1}\left(r_{-(m-1)}^{k}\right)} \\
=-\sum_{a=1}^{m-1} \frac{v\left(F^{a-1}\left(r_{-(m-1)}^{k}\right)\right)}{D F^{a}\left(r_{-(m-1)}^{k}\right)}+v\left(r_{-m}^{k}\right)+\sum_{a=1}^{m-1} \frac{v\left(F^{a-1}\left(r_{-(m-1)}^{k}\right)\right)}{D F^{a}\left(r_{-(m-1)}^{k}\right)} \\
=v\left(r_{-m}^{k}\right) .
\end{gathered}
$$

So (66) holds for every $z \in \Gamma$. Since $v, \alpha$ and $F$ are continuous in a neighbourhood of $P(F)$ and $\Gamma$ is dense in $P(F)$, it follows that (66) holds for every $z \in P(F)$.

Corollary 7.3 (Induced problem). Let $F \in \Omega_{n, p}$ and $v \in \mathfrak{B}_{+}(F)$. If there exists a quasiconformal vector field $\alpha$, defined in a neighbourhood of $P(F)$, such that $\alpha(r)=0$ for every $r \in C(F)$ and

$$
V(z)=\alpha \circ G(z)-D G(z) \cdot \alpha(z)
$$

for every $z \in P(F) \backslash C(F)$, then $v \in E^{h}(F)$.

Proof. This is an immediate consequence of Proposition 4.1 and Proposition 7.2.

\section{Solving the INDUCED PROBlem.}

We are going to change a little bit the notation. Let $P_{i}^{k}, i=1, \ldots, n$ be the set of restrictive intervals for $F$ associated to the renomalization $\mathcal{R}^{k} F$, that is

A. These intervals are pairwise disjoint, $C(F) \cap P_{i}^{k} \neq \emptyset$ and

$$
C(F) \subset \cup_{i} P_{i}^{k} .
$$

B. There are integers $n_{i}^{k}$ such that

$$
F^{n_{i}^{k}}: P_{i}^{k} \rightarrow P_{i+1}^{k} \bmod n
$$

is an unimodal map.

C. The have that $P_{i}^{k}=\left[\delta_{i}^{k}, b_{i}^{k}\right]$, where $\delta_{i}^{k}$ is a $\mu^{(k)}$-periodic repelling fixed point, with

$$
\mu^{(k)}=\sum_{i} n_{i}^{k}
$$

and $F^{n_{i}^{k}}\left(\delta_{i}^{k}\right)=F^{n_{i}^{k}}\left(b_{i}^{k}\right)$.

D. The renormalization associated to the restrictive interval $P_{1}^{k}$ is $\mathcal{R}^{k} F$. 
If $t$ is small enough then $F_{t}=F+t v$ is close to $F$ and $\delta_{i}^{k}$ has an analytic continuation $\delta_{i}^{k}(t)$. Denote by $\partial_{t} \delta_{i}^{k}$ the derivative of this continuation with respect to $t$ at $t=0$. Consider $\left\{q_{i}^{k}\right\}=C(F) \cap P_{i}^{k}$. Then $q_{i+1}^{k}$ is the successor of $q_{i}^{k}$ at level $k$. Let $j_{i}^{k} \in\{1, \ldots, n\}$ be such that $q_{i}^{k} \in I_{j_{i}^{k}}$, and

$$
A_{i}^{k}: \mathbb{C} \times\left\{j_{i}^{k}\right\} \rightarrow \mathbb{C} \times\{i\}
$$

be the only affine transformation such that $A_{i}^{k}\left(\delta_{i}^{k}\right)=(-1, i)$ and $A_{i}^{k}\left(q_{i}^{k}\right)=(0, i)$.

Lemma 8.1. We have $V=V_{1}+V_{2}$, where

$$
V_{1}\left(z, j_{i}^{k}\right)=-\delta_{i+1}^{k} v^{k} \circ A_{i}^{k}\left(z, j_{i}^{k}\right),
$$

and

$$
V_{2}\left(z, j_{i}^{k}\right)=\frac{\partial_{t} \delta_{i+1}^{k}}{\delta_{i+1}^{k}} F^{n_{i}^{k}}\left(z, j_{i}^{k}\right)-D F^{n_{i}^{k}}\left(z, j_{i}^{k}\right) \cdot \frac{\partial_{t} \delta_{i}^{k}}{\delta_{i}^{k}}\left(z, j_{i}^{k}\right)
$$

for every $\left(z, j_{i}^{k}\right) \in \mathscr{R}_{-\ell}^{k+1}$, with $R_{-\ell}^{k+1} \subset Q_{0}^{k}, q=q_{i}^{k}$.

Proof. Note that

$$
\begin{gathered}
v^{k}(x, i)=\left.\partial_{t}\left(\mathcal{R}^{k}\left(F_{t}\right)\right)\right|_{t=0}(x, i)=\left(D \mathcal{R}_{F}^{k} \cdot v\right)(x, i) \\
=-\frac{\partial_{t} \delta_{i+1}^{k}}{\delta_{i+1}^{k}} \cdot A_{i+1}^{k} \circ F^{n_{i}^{k}} \circ\left(A_{i}^{k}\right)^{-1}(x, i) \\
-\frac{1}{\delta_{i+1}^{k}}\left(a_{n_{i}^{k}} \circ\left(A_{i}^{k}\right)^{-1}(x, i)+\left(D F^{n_{i}^{k}}\right) \circ\left(A_{i}^{k}\right)^{-1}(x, i) \cdot\left(-\partial_{t} \delta_{i}^{k} x, i\right)\right),
\end{gathered}
$$

So if $(x, i)=A_{i}^{k}\left(z, j_{i}^{k}\right)$, with $\left(z, j_{i}^{k}\right) \in \mathscr{R}_{-\ell}^{k+1}$, with $R_{-\ell}^{k+1} \subset Q_{0}^{k}$ we have that

$$
\begin{gathered}
V\left(z, j_{i}\right)=a_{n_{i}^{k}}\left(z, j_{i}^{k}\right) \\
=-\delta_{i+1}^{k} v^{k} \circ A_{i}^{k}\left(z, j_{i}^{k}\right)+\frac{\partial_{t} \delta_{i+1}^{k}}{\delta_{i+1}^{k}} F^{n_{i}^{k}}\left(z, j_{i}^{k}\right)-D F^{n_{i}^{k}}\left(z, j_{i}^{k}\right) \cdot \frac{\partial_{t} \delta_{i}^{k}}{\delta_{i}^{k}}\left(z, j_{i}^{k}\right) .
\end{gathered}
$$

Lemma 8.2. Let $v \in \mathfrak{B}_{+}(F)$. There exists $C_{3}>0$ such that for every $k$ and every $i_{k}, i_{k+1}$ such that $q_{i_{k}}^{k}=q_{i_{k+1}}^{k+1}$ we have

$$
\left|\frac{\partial_{t} \delta_{i_{k+1}}^{k+1}}{\delta_{i_{k+1}}^{k+1}}-\frac{\partial_{t} \delta_{i_{k}}^{k}}{\delta_{i_{k}}^{k}}\right| \leq C_{3}
$$

Proof. Let $q=q_{i_{k}}^{k}=q_{i_{k+1}}^{k+1}$ and $i_{j}$ be such that $q_{i_{j}}^{j}=q$. Note that $\beta_{q}^{k+1}=A_{i}^{k}\left(\delta_{i_{k+1}}^{k+1}\right)$ is a periodic point for $\mathcal{R}^{k} F$ with period $y^{k}=\mu^{(k+1)} / \mu^{(k)}$. Indeed

$$
\beta_{q}^{k+1}=\left(\frac{\delta_{i_{k+1}}^{k+1}}{\delta_{i_{k}}^{k}}, i_{k}\right) .
$$

If $t$ is small enough then there is an analytic continuation $\beta_{q}^{k+1}(t)$ for $\beta_{q}^{k+1}$, that is a periodic point for $\mathcal{R}^{k} F_{t}$. Since

$$
\left.\partial_{t}\left(\mathcal{R}^{k}\left(F_{t}\right)\right)\right|_{t=0}(x, i)=\left(D \mathcal{R}_{F}^{k} \cdot v\right)(x, i)=v^{k}(x, i),
$$

by the Implicit Function Theorem we have that

$$
\left.\partial_{t}\left(\mathcal{R}^{k}\left(F_{t}\right)\right)^{y^{k}}\right|_{t=0}\left(\beta_{q}^{k+1}\right)+D\left(\mathcal{R}^{k} F\right)^{y^{k}}\left(\beta_{q}^{k+1}\right) \partial_{t} \beta_{q}^{k+1}=\partial_{t} \beta_{q}^{k+1} .
$$


So

$$
\begin{gathered}
\partial_{t} \beta_{q}^{k+1} \\
=\frac{1}{1-D\left(\mathcal{R}^{k} F\right)^{y^{k}}\left(\beta_{q}^{k+1}\right)} \sum_{j=0}^{y^{k}-1} D\left(\mathcal{R}^{k} F\right)^{y^{k}-j-1}\left(\left(\mathcal{R}^{k} F\right)^{j+1}\left(\beta_{q}^{k+1}\right)\right) v^{k}\left(\left(\mathcal{R}^{k} F\right)^{j}\left(\beta_{q}^{k+1}\right)\right) \\
=\sum_{\ell=1}^{\infty} \frac{-1}{D\left(\mathcal{R}^{k} F\right)^{y^{k} \ell}\left(\beta_{q}^{k+1}\right)} \sum_{j=0}^{y^{k}-1} D\left(\mathcal{R}^{k} F\right)^{y^{k}-j-1}\left(\left(\mathcal{R}^{k} F\right)^{j+1}\left(\beta_{q}^{k+1}\right)\right) v^{k}\left(\left(\mathcal{R}^{k} F\right)^{j}\left(\beta_{q}^{k+1}\right)\right) \\
=\sum_{\ell=1}^{\infty} \sum_{j=0}^{y^{k}-1}-\frac{v^{k}\left(\left(\mathcal{R}^{k} F\right)^{y^{k}(\ell-1)+j}\left(\beta_{q}^{k+1}\right)\right)}{D\left(\mathcal{R}^{k} F\right)^{y^{k}(\ell-1)+j+1}\left(\beta_{q}^{k+1}\right)} \\
=\sum_{a=0}^{\infty}-\frac{v^{k}\left(\left(\mathcal{R}^{k} F\right)^{a}\left(\beta_{q}^{k+1}\right)\right)}{D\left(\mathcal{R}^{k} F\right)^{a+1}\left(\beta_{q}^{k+1}\right)}
\end{gathered}
$$

Due the real bounds and $v \in \mathfrak{B}_{+}(F)$ there exist $C_{1}, C_{2}$ such that

$$
\sup _{k}\left\{\left|D\left(\mathcal{R}^{k} F\right)\right|_{\mathcal{B}(V)},\left|\beta_{q}^{k}\right|, \frac{1}{\left|\beta_{q}^{k}\right|},\left|v^{k}\right|_{\mathcal{B}(V)}\right\} \leq C_{1}
$$

and

$$
1<C_{2}<\inf _{k, q}\left|D\left(\mathcal{R}^{k} F\right)^{y^{k}}\left(\beta_{q}^{k+1}\right)\right| .
$$

So

$$
\sup _{k, q}\left|\frac{\partial_{t} \beta_{q}^{k+1}}{\beta_{q}^{k+1}}\right|=C_{3}<\infty
$$

If $i_{j}$ satisfies $q_{i_{j}}^{j}=q$ then

$$
\delta_{i_{k+1}}^{k+1}(t)=\prod_{j=0}^{k} \frac{\delta_{i_{j+1}}^{j+1}(t)}{\delta_{i_{j}}^{j}(t)}=\prod_{j=1}^{k+1} \beta_{q}^{j}(t),
$$

if we derive with respect to $t$ at $t=0$ we obtain

$$
\partial_{t} \delta_{i_{k+1}}^{k+1}=\sum_{j=1}^{k+1} \partial_{t} \beta_{q}^{j} \prod_{\ell \neq j} \beta_{q}^{\ell}
$$

and we conclude that

$$
\frac{\partial_{t} \delta_{i_{k+1}}^{k+1}}{\delta_{i_{k+1}}^{k+1}}=\sum_{j=1}^{k+1} \frac{\partial_{t} \beta_{q}^{j}}{\beta_{q}^{j}}
$$

and

$$
\left|\frac{\partial_{t} \delta_{i_{k+1}}^{k+1}}{\delta_{i_{k+1}}^{k+1}}-\frac{\partial_{t} \delta_{i_{k}}^{k}}{\delta_{i_{k}}^{k}}\right|=\left|\frac{\partial_{t} \beta_{q}^{k+1}}{\beta_{q}^{k+1}}\right| \leq C_{3}
$$


Lemma 8.3. For every $\left(z, j_{i}^{k}\right) \in \mathscr{R}_{-\ell}^{k+1}$, with $R_{-\ell}^{k+1} \subset Q_{0}^{k}, q=q_{i}^{k}$ we have

$$
\left|V_{1}\left(z, j_{i}^{k}\right)\right| \leq\left|\delta_{i+1}^{k}\right| \sup _{k}\left|v^{k}\right|
$$

and

$$
\left|V_{2}\left(z, j_{i}^{k}\right)\right| \leq C_{3} k\left|\delta_{i+1}^{k}\right|+C_{3} k\left|\delta_{i}^{k}\right| \sup |D G|
$$

In particular

$$
\lim _{w \rightarrow C(F)} V(w)=0 .
$$

Proof. It follows from Lemma 8.1 and Lemma 8.2.

Lemma 8.4. Let $\psi: \mathbb{R}_{+}^{\star} \rightarrow \mathbb{R}$ be a $C^{\infty}$ function. Define $\gamma: \mathbb{C} \backslash\{0\} \rightarrow \mathbb{C}$ as

$$
\gamma(z)=\psi(|z|) z .
$$

Then

$$
|\bar{\partial} \gamma(z)|=\frac{|z D \psi(|z|)|}{2}
$$

If $|D \psi(t)| \leq C t^{-1}$ then $\gamma$ can be extended as a quasiconformal vector field on $\mathbb{C}$ such that $\gamma(0)=0$.

Proof. If $z=x+i y, x, y \in \mathbb{R}$ then

$$
\psi(|z|)=\psi\left(\sqrt{x^{2}+y^{2}}\right),
$$

In particular

$$
\bar{\partial}(\psi(|z|))=\frac{2 x D \psi\left(\sqrt{x^{2}+y^{2}}\right)}{4 \sqrt{x^{2}+y^{2}}}+i \frac{2 y D \psi\left(\sqrt{x^{2}+y^{2}}\right)}{4 \sqrt{x^{2}+y^{2}}},
$$

and

In particular

$$
|\bar{\partial}(\psi(|z|))|=\frac{|D \psi(|z|)|}{2} .
$$

$$
|\bar{\partial}(\psi(|z|) z)|=\frac{|z D \psi(|z|)|}{2} .
$$

Let $\epsilon \in(0,1)$. Note that

In particular

$$
\begin{gathered}
|\psi(1)-\psi(\epsilon)|=\left|\int_{\epsilon}^{1} D \psi(t) d t\right| \leq \int_{\epsilon}^{1} \frac{1}{t} d t=-\ln \epsilon . \\
|\psi(\epsilon)| \leq-\ln \epsilon+|\psi(1)| .
\end{gathered}
$$

$$
\lim _{z \rightarrow 0} z \psi(|z|)=0
$$

so defining $\gamma(0)=0$ we obtain a continuous extension to $\mathbb{C}$ of $\gamma$. To show that $\gamma$ is a quasiconformal vector field, note that

$$
|\bar{\partial} \gamma(z)|=\frac{\mid z D \psi(|z|)}{2} \leq \frac{C}{2}
$$

for every $z \in \mathbb{C} \backslash\{0\}$. By [1, Lemma 3, page 53] there exists a quasiconformal vector field $\tilde{\gamma}$ on $\mathbb{C}$ such that its distributional derivative belongs to $L^{2}(\mathbb{C})$, it satisfies $\bar{\partial} \tilde{\gamma}(z)=0$ if $|z| \geq 1$ and

$$
\bar{\partial} \tilde{\gamma}(z)=\bar{\partial} \gamma(z)
$$


for almost every $z$ satisfying $|z|<1$. So $\gamma-\tilde{\gamma}$ is continuous on $\{z \in \mathbb{C}$ : $|z|<1\}$ and (by Weyl's Lemma) holomorphic on $\{z \in \mathbb{C}$ : $0<|z|<1\}$. By Riemann's Theorem on removable singularities we have that 0 is a removable singularity, so $\gamma-\tilde{\gamma}$ is holomorphic on $|z|<1$, and therefore $\gamma$ is quasiconformal on $\mathbb{C}$.

An illustrative example of application of Lemma 8.4 is obtained considering $\psi(x)=\log (x)$.

Proposition 8.5. Let $v \in \mathfrak{B}_{+}(F)$. There exists a quasiconformal vector field $\alpha_{2}: \mathbb{C}_{n} \rightarrow \mathbb{C}$ such that

$$
V_{2}(z, j)=\alpha_{2} \circ G(z, j)-D G(z, j) \cdot \alpha_{2}(z, j)
$$

for every $(z, j) \in \partial \mathscr{D}$ and moreover $\alpha_{2}(0, j)=0$ for every $j$.

Proof. Due the real bounds we have that

$$
\inf _{q \in C(F)} \inf _{k} \min _{R_{-\ell}^{k+1} \subset Q_{0}^{k}} \frac{\operatorname{dist}\left(R_{-\ell}^{k+1}, \partial Q_{0}^{k}\right)}{\left|Q_{0}^{k}\right|}=\epsilon_{1}>0 .
$$

Without loss of generality we can choose $\epsilon_{0}$ in (60) satisfiying $\epsilon_{0}<\epsilon_{1} / 4$. Let $\phi: \mathbb{R} \rightarrow \mathbb{R}$ be a $C^{\infty}$ function such that

i. $\phi(x) \in[0,1]$ for every $x \in \mathbb{R}$.

ii. If $|x|<1-\epsilon_{1} / 4$ then $\phi(x)=1$.

iii. If $|x|>1-\epsilon_{1} / 8$ then $\phi(x)=0$.

Given $q \in C(F)$, let $i_{j}$ be such that $q_{i_{j}}^{j}=q$ and $\delta_{i_{j}}^{j}$ and $\beta_{q}^{j}, j \in \mathbb{N}$, be as in the proof of Lemma 8.2. For every $x \in \mathbb{R}^{\star}$ define

$$
\psi_{q}(x)=\sum_{j=1}^{\infty} \frac{\partial_{t} \beta_{q}^{j}}{\beta_{q}^{j}} \cdot \phi\left(\frac{x}{\delta_{i_{j}}^{j}}\right)
$$

The function $\psi_{q}$ is well defined in $\mathbb{R} \backslash\{0\}$, it is $C^{\infty}$ on $\mathbb{R} \backslash\{0\}$ and if

$$
\left(1-\epsilon_{1} / 8\right)\left|\delta_{i_{k+1}}^{k+1}\right| \leq|x| \leq\left(1-\epsilon_{1} / 2\right)\left|\delta_{i_{k}}^{k}\right|
$$

then

$$
\psi_{q}(x)=\sum_{j=1}^{k} \frac{\partial_{t} \beta_{q}^{j}}{\beta_{q}^{j}} \cdot \phi\left(\frac{x}{\delta_{i_{j}}^{j}}\right)=\sum_{j=1}^{k} \frac{\partial_{t} \beta_{q}^{j}}{\beta_{q}^{j}}=\frac{\partial_{t} \delta_{i_{k}}^{k}}{\delta_{i_{k}}^{k}} .
$$

Moreover, notice that if

$$
\left|\delta_{i_{k+1}}^{k+1}\right| \leq|x| \leq\left|\delta_{i_{k}}^{k}\right|
$$

then

$$
\psi_{q}(x)=\frac{\partial_{t} \beta_{q}^{k}}{\beta_{q}^{k}} \cdot \phi\left(\frac{x}{\delta_{i_{k}}^{k}}\right)+\sum_{j=1}^{k-1} \frac{\partial_{t} \beta_{q}^{j}}{\beta_{q}^{j}}
$$

So

$$
D \psi_{q}(x)=\frac{\partial_{t} \beta_{q}^{k}}{\delta_{i_{k}}^{k} \beta_{q}^{k}} \cdot D \phi\left(\frac{x}{\delta_{i_{k}}^{k}}\right)
$$

and by (72) and (82)

$$
\left|D \psi_{q}(x)\right| \leq \frac{1}{\left|\delta_{i_{k}}^{k}\right|} C_{3} \max |D \phi| \leq \frac{C_{3} \max |D \phi|}{|x|}
$$


If $q=(0, j)$, define

$$
\alpha_{2}(z, j)=\psi_{q}(|z|) z
$$

By (83) and Lemma 8.4 we conclude that $\alpha_{2}$ is a quasiconformal vector field with $\alpha_{2}(0, j)=0$ for every $j$.

It remains to show that $\alpha_{2}$ satisfies (78). Indeed, let $(z, j) \in \partial \mathscr{R}_{-\ell}^{k+1}$, with $R_{-\ell}^{k+1} \subset Q_{0}^{k}$ and $\ell>0$. Since $\mathscr{R}_{-\ell}^{k+1}$ belongs to a disc with diameter given by the interval $R_{-\ell}^{k+1}$ and it does not intercept $\mathcal{Q}_{0}^{k+1}$, we have

$$
\left(1-\epsilon_{1} / 64\right)\left|\delta_{i_{k+1}}^{k+1}\right| \leq d_{q}^{k+1} \leq|z| \leq\left(1-\epsilon_{1}\right)\left|\delta_{i_{k}}^{k}\right| .
$$

for every $(z, j) \in \partial \mathscr{R}_{-\ell}^{k+1}$. Here $d_{q}^{k+1}$ is as defined in (61). By (81) we have that

$$
\alpha_{2}(z, j)=\frac{\partial_{t} \delta_{i_{k}}^{k}}{\delta_{i_{k}}^{k}} z
$$

for every

$$
(z, j) \in \partial \mathscr{R}_{-\ell}^{k+1} \text {. }
$$

Indeed, let $(z, \tilde{\mathrm{j}}) \in \mathscr{R}_{-\ell}^{k+1}$, with $R_{-\ell}^{k+1} \subset S_{0}^{k}$, with $\ell>0$ and such that $q$ is the sucessor of $s$ at level $k$. Then

$$
G\left(\mathscr{R}_{-\ell}^{k+1}\right) \subset \mathcal{Q}_{0}^{k} .
$$

If $G\left(\mathscr{R}_{-\ell}^{k+1}\right)=\mathscr{R}_{-a}^{k+1}$, for some $a>0$, then the points in this image also satisfies (85). Otherwise $r=q$ and $G\left(\mathscr{R}_{-\ell}^{k+1}\right)=\mathcal{Q}_{0}^{k+1}$, so if $(w, j) \in G\left(\partial \mathscr{R}_{-\ell}^{k+1}\right)$ then

$$
\left(1-\epsilon_{1} / 64\right)\left|\delta_{i_{k+1}}^{k+1}\right| \leq\left|d_{q}^{k+1}\right|=|w| \leq\left|\delta_{i_{k+1}}^{k+1}\right| \leq\left(1-\epsilon_{1}\right)\left|\delta_{i_{k}}^{k}\right| .
$$

By (81) we have that

$$
\alpha_{2}(w, j)=\frac{\partial_{t} \delta_{i_{k}}^{k}}{\delta_{i_{k}}^{k}} w
$$

for every

$$
(w, j) \in G\left(\partial \mathscr{R}_{-\ell}^{k+1}\right),
$$

Since these estimates holds for every $q \in C(F)$ we have that for every $(z, j) \in$ $\partial \mathscr{R}_{-\ell}^{k+1}$, with $R_{-\ell}^{k+1} \subset Q_{0}^{k}$ and $\ell>0$ we have

$$
\begin{aligned}
\alpha_{2} \circ G(z, j)-D G(z, j) \cdot \alpha_{2}(z, j) & =\frac{\partial_{t} \delta_{i_{k}+1}^{k}}{\delta_{i_{k}+1}^{k}} G(z, j)-D G(z, j) \frac{\partial_{t} \delta_{i_{k}}^{k}}{\delta_{i_{k}}^{k}}(z, j) \\
& =\frac{\partial_{t} \delta_{i_{k}+1}^{k}}{\delta_{i_{k}+1}^{k}} F^{n_{i_{k}}^{k}}(z, j)-D F^{n_{i_{k}}^{k}}(z, j) \frac{\partial_{t} \delta_{i_{k}}^{k}}{\delta_{i_{k}}^{k}}(z, j) \\
& =V_{2}(z, j) .
\end{aligned}
$$

Proposition 8.6. Let $v \in \mathfrak{B}_{+}(F)$. There exists a quasiconformal vector field $\alpha_{1}: \mathbb{C}_{n} \rightarrow \mathbb{C}$ such that

$$
V_{1}(z, j)=\alpha_{1} \circ G(z, j)-D G(z, j) \cdot \alpha_{1}(z, j)
$$

for every $(z, j) \in \partial \mathscr{D}$ and moreover $\alpha_{1}(q)=0$ for every $q \in C(F)$.

Proof. Let $\epsilon_{1}>0$ be as in (79). Let $\phi: \mathbb{C} \rightarrow \mathbb{R}$ be a $C^{\infty}$ function such that

i. $\phi(x) \in[0,1]$ for every $x \in \mathbb{C}$. 
ii. If $|x|=1-\epsilon_{0} / 16$ and then $\phi(x)=1$.

iii. If either $|x|>1-\epsilon_{0} / 32,|x|<1-\epsilon_{1} / 2$ then $\phi(x)=0$.

Given $r \in C(F)$, let

$$
i_{0}=0<i_{1}<\cdots<i_{\ell_{r}^{k}}<n_{r}^{k}
$$

be the sequence of integers $i<n_{r}^{k}$ such that

$$
R_{i}^{k} \subset \bigcup_{q \in C(F)} Q_{0}^{k-1}
$$

Denote by $\delta_{r}^{k}$ the periodic point in the boundary of $R_{0}^{k}$. There is an onto univalent extension

$$
G^{j}: D_{r,-j}^{k} \rightarrow B\left(r,\left|\delta_{r}^{k}\right|\right),
$$

where $D_{r,-j}^{k} \cap \mathbb{R}=R_{-i_{j}}^{k}$. Note that $\mathscr{R}_{-i_{j}}^{k} \subset D_{r,-j}^{k}$ and

$$
G^{j}\left(\mathscr{R}_{-i_{j}}^{k}\right)=B\left(r,\left(1-\epsilon_{0} / 16\right)\left|\delta_{r}^{k}\right|\right)=\mathscr{R}_{0}^{k} .
$$

Let $q_{j} \in C(F)$ such that $q_{j}$ and $R_{-i_{j}}^{k}$ belongs to the same connected component of $I_{F}^{n}$. Define a function

$$
\psi_{r,-j}^{k}: \mathbb{C}_{n} \rightarrow \mathbb{C}
$$

in the following way. Let

$$
\psi_{r, 0}^{k}(z, a)=0
$$

for every $(z, a) \in \mathbb{C}_{n}$, and define by induction on $j$

$$
\psi_{r,-(j+1)}^{k}(z, a)=\phi\left(\frac{G^{j+1}(z, a)}{\delta_{r}^{k}}\right) \frac{\psi_{r,-j}^{k}(G(z, a))-\delta_{q_{j}}^{k-1} v^{k-1} \circ A_{q_{j+1}}^{k-1}(z, a)}{D G(z, a)}
$$

for every $(z, a) \in D_{r,-(j+1)}^{k}$. Note that $\psi_{r,-(j+1)}^{k}(z, a)=0$ for $(z, a)$ in a neighbourhood of $\partial D_{r,-(j+1)}^{k}$, so we can extend $\psi_{r,-(j+1)}^{k}$ to a $C^{\infty}$ function on $\mathbb{C}_{n}$ defining $\psi_{r,-(j+1)}^{k}(z, a)=0$ for $(z, a) \notin D_{r,-(j+1)}^{k}$. Finally note that by $(89)$

$$
\phi\left(\frac{G^{j+1}(z, a)}{\delta_{r}^{k}}\right)=1
$$

for every $(z, a) \in \partial \mathscr{R}_{-i_{j+1}}^{k}$, so by $(90)$

$$
\text { (91) }-V_{1}(z, a)=\delta_{q_{j}}^{k-1} v^{k-1} \circ A_{q_{j+1}}^{k-1}(z, a)=\psi_{r,-j}^{k}(G(z, a))-D G(z, a) \psi_{r,-(j+1)}^{k}(z, a)
$$

for every $(z, a) \in \partial \mathscr{R}_{-i_{j}}^{k}$. Note that given $(z, a) \in \mathbb{C}_{n} \backslash C(F)$, there exists an open neighbourhood of $(z, a)$ that intersects only one of the supports of a function in the family

$$
\mathcal{F}=\left\{\psi_{r,-j}^{k}\right\}_{k, r \in C(F), j \leq \ell_{r}^{k}}
$$

In particular the function

$$
\alpha_{1}(z, a)=-\sum_{k} \sum_{r \in C(F)} \sum_{j=0}^{\ell_{r}^{k}} \psi_{r,-j}^{k}(z, a)
$$

is well defined and it is $C^{\infty}$ on $\mathbb{C}_{n} \backslash C(F)$. Moreover given $\mathscr{R}_{-i_{j}}^{k}$, the function $\psi_{r,-j}^{k}$ is the unique function in this family whose support intersects $\partial \mathscr{R}_{-i_{j}}^{k}$. By (91), this implies that

$$
\left.V_{1}(z, a)=\alpha_{1} \circ G(z, a)\right)-D G(z, a) \alpha_{1}(z, a) .
$$


for every $(z, a) \in \partial \mathscr{D}$. It is easy to prove by induction that

$$
\left|\psi_{r,-j}^{k}(z, a)\right| \leq \sup _{k}\left|v^{k-1}\right| \sum_{\ell=0}^{j-1} \frac{\left|\delta_{q_{j-1-\ell}}^{k-1}\right|}{|D G|^{\ell+1}(z, a)},
$$

in particular

$$
\left|\psi_{r,-j}^{k}\right| \leq \max _{q \in C(F)}\left|\delta_{q}^{k-1}\right| \sup _{k}\left|v^{k-1}\right| \sum_{j=0}^{\sup _{k} \frac{p_{k}}{p_{k-1}}} \frac{1}{(\inf |D G|)^{j}} .
$$

Due the real bounds

$$
\left|\psi_{r,-j}^{k}\right| \leq C \theta^{-k}
$$

If $(z, a) \in \mathcal{Q}_{0}^{k-1} \backslash\{q\}$, for some $q \in C(F)$ then either $\alpha_{1}(z, a)=0$ or $(z, a)$ belongs to the support of a unique function $\psi_{r,-j}^{b}$, with $b \geq k$. We conclude that

$$
\lim _{(z, a) \rightarrow q} \alpha_{1}(z, a)=0,
$$

so we can extend $\alpha_{1}$ as a continuous function on $\mathbb{C}_{n}$. Note that

$$
\begin{aligned}
& \bar{\partial} \psi_{r,-(j+1)}^{k}(z, a) \\
= & \bar{\partial} \phi\left(\frac{G^{j+1}(z, a)}{\delta_{r}^{k}}\right) \frac{\overline{D G^{j+1}}(z, a)}{\overline{\delta_{r}^{k}}} \frac{\psi_{r,-j}^{k}(G(z, a))-\delta_{q_{j}}^{k-1} v^{k-1} \circ A_{q_{j+1}}^{k-1}(z, a)}{D G(z, a)} \\
(96)+ & \phi\left(\frac{G^{j+1}(z, a)}{\delta_{r}^{k}}\right) \bar{\partial} \psi_{r,-j}^{k}(G(z, a)) \frac{\overline{D G}(z, a)}{D G(z, a)}
\end{aligned}
$$

By the real bounds there exists $C>0$ such that

$$
\frac{\left|D G^{j-\ell}\right|\left(G^{\ell+1}(z, a)\right)}{\left|\delta_{r}^{k}\right|} \leq \frac{C}{\left|R_{-i_{j-\ell}}^{k}\right|}
$$

for every $r \in C(F), K$ and $j$ and $(z, a) \in D_{r,-(j+1)}^{k}$. In particular

$$
\begin{aligned}
& \frac{\left|D G^{j+1}\right|(z, a)}{\left|\delta_{r}^{k}\right|} \frac{\left|\psi_{r,-j}^{k}(G(z, a))-\delta_{q_{j}}^{k-1} v^{k-1} \circ A_{q_{j+1}}^{k-1}(z, a)\right|}{|D G(z, a)|} \\
\leq & \frac{\left|D G^{j+1}\right|(z, a)}{\left|\delta_{r}^{k}\right|}\left(\sum_{\ell=0}^{j-1} \frac{\left|\delta_{q_{j-1-\ell}}^{k-1}\right|}{|D G|^{\ell+2}(z, a)}+\frac{\left|\delta_{q_{j}}^{k-1}\right|}{|D G|(z, a)}\right) \\
\leq & \frac{\left|D G^{j+1}\right|(z, a)}{\left|\delta_{r}^{k}\right|} \sum_{\ell=0}^{j} \frac{\left|\delta_{q_{j-\ell}}^{k-1}\right|}{|D G|^{\ell+1}(z, a)} \\
\leq & \sum_{\ell=0}^{j} \frac{\left|D G^{j-\ell}\right|\left(G^{\ell+1}(z, a)\right)\left|\delta_{q_{j-\ell}}^{k-1}\right|}{\left|\delta_{r}^{k}\right|} \leq C \sum_{\ell=0}^{j} \frac{\left|\delta_{q_{j-\ell}}^{k-1}\right|}{\left|R_{-i_{j-\ell}}^{k}\right|} \\
\leq & C \sup _{k} \frac{\mu^{(k)}}{\mu^{(k-1)}} .
\end{aligned}
$$

The last inequality follows from the fact that $R_{-i_{j-\ell}}^{k} \subset Q_{0}^{k-1}$, with $q=q_{j-\ell}$, and $2 \delta_{q_{j-\ell}}^{k-1}=\left|Q_{0}^{k-1}\right|$ and by the real bounds there exists $C>0$ such that

$$
\frac{\left|Q_{0}^{k-1}\right|}{\left|R_{-i}^{k}\right|} \leq C
$$


for every $R_{-i}^{k} \subset Q_{0}^{k-1}$. So by (97)

$$
\sup \left|\bar{\partial} \psi_{r,-(j+1)}^{k}\right| \leq C \sup |\bar{\partial} \phi| \sup _{k} \frac{\mu^{(k)}}{\mu^{(k-1)}}+\sup \left|\bar{\partial} \psi_{r,-j}^{k}\right| .
$$

Since $\psi_{0}^{k}=0$ we obtain

$$
\sup \left|\bar{\partial} \psi_{r,-j}^{k}\right| \leq C \sup _{k} \frac{\mu^{(k)}}{\mu^{(k-1)}}
$$

Since for every $(z, a) \in \mathbb{C}_{n} \backslash C(F)$, there exists an open neighbourhood of $(z, a)$ that intersects only one of the supports of the functions in the family $\mathcal{F}$, we conclude that $\alpha_{1}$ is a quasiconformal vector field on $\mathbb{C}_{n} \backslash C(F)$. Since $\alpha_{1}$ is continuous at $C(F)$, we can use the argument in the end of the proof of Lemma 8.4 to conclude that $\alpha_{1}$ is a quasiconformal vector field on $\mathbb{C}_{n}$.

Proof of Theorem 4. Let $v \in \mathfrak{B}_{+}(F)$. Let $\alpha_{1}$ and $\alpha_{2}$ as in Proposition 8.5 and 8.6. Define $\alpha^{0}=\alpha_{1}+\alpha_{2}$. Then by Lemma 8.1

$$
V(z, j)=\alpha^{0} \circ G(z, j)-D G(z, j) \cdot \alpha^{0}(z, j)
$$

for every $(z, j) \in \partial \mathscr{D} \backslash C(F)$ and moreover $\alpha^{0}(q)=0$ for every $q \in C(F)$. Now we will use an argument similar to the infinitesimal pullback argument for polynomiallike maps [3]. We define by induction on $m$ a sequence of quasiconformal vector fields

$$
\alpha^{m}: \mathbb{C}_{n} \rightarrow \mathbb{C}
$$

such that $\alpha^{m}(q)=0$ for every $q \in C(F)$,

$$
V(z, j)=\alpha^{m} \circ G(z, j)-D G(z, j) \cdot \alpha^{m}(z, j)
$$

for every $(z, j) \in \partial \mathscr{D}$ and moreover

$$
V(z, j)=\alpha^{m-1} \circ G(z, j)-D G(z, j) \cdot \alpha^{m}(z, j)
$$

for every $(z, j) \in \mathscr{D}, m \geq 1$ and

$$
\sup _{\mathbb{C}_{n}}\left|\bar{\partial} \alpha^{m+1}\right| \leq \sup _{\mathbb{C}_{n}}\left|\bar{\partial} \alpha^{m}\right|
$$

Indeed, suppose by induction we have defined $\alpha^{k}$. Define $\alpha^{k+1}(z, j)=\alpha^{k}(z, j)$ for every $(z, j) \notin \mathscr{D}$ and

$$
\alpha^{k+1}(z, j)=\frac{\alpha^{k}(G(z, j))-V(z, j)}{D G(z, j)}
$$

for every $(z, j) \in \mathscr{D}$. Note that

$$
\left|\bar{\partial} \alpha^{k+1}(z, j)\right|=\left|\bar{\partial} \alpha^{k}(z, j)\right|,
$$

for every $(z, j) \notin \overline{\mathscr{D}}$ and

$$
\left|\bar{\partial} \alpha^{k+1}(z, j)\right|=\left|\bar{\partial} \alpha^{k}(G(z, j))\right|
$$

for $(z, j) \in \mathscr{D}$. So $\alpha^{k+1}$ is a quasiconformal vector field in $\mathbb{C}_{n} \backslash \partial \mathscr{D}$. Moreover (101) holds for $m=k+1$ and due (100) with $m=k$ we have that $\alpha^{k+1}=\alpha^{k}$ on $\partial \mathscr{D} \backslash C(F)$, so $\alpha^{k+1}$ is continuous at points in

$$
\partial \mathscr{D} \backslash C(F) \text {. }
$$


If $(z, j) \in \partial \mathscr{D} \backslash C(F)$ then $(z, j) \in \partial \mathscr{R}_{-i}^{n}$, for some $r \in C(F)$. In particular there exists a neighbourhood $W$ of $(z, j)$ such that $\alpha^{k+1}$ is continuous on $W$ and a quasiconformal vector field on $W \backslash \partial \mathscr{R}_{-i}^{n}$. Since $\partial \mathscr{R}_{-i}^{n}$ is an analytic curve we conclude that $\alpha^{k+1}$ is a quasiconformal vector field on $W$. So $\alpha^{k+1}$ is a quasiconformal vector field on $\mathbb{C}_{n} \backslash C(F)$. Finally notice that $\alpha^{k+1}$ is continuous at points in $C(F)$. Indeed, suppose that

$$
\left(z_{\ell}, i\right) \rightarrow_{\ell}(0, i)
$$

If $\left(z_{\ell}, i\right) \notin \mathscr{D}$ for every $\ell$ then

$$
\lim _{\ell \rightarrow \infty} \alpha^{k+1}\left(z_{\ell}, i\right)=\lim _{\ell \rightarrow \infty} \alpha^{k}\left(z_{\ell}, i\right)=0 .
$$

If $\left(z_{\ell}, i\right) \in \mathscr{D}$ for every $\ell$ then

$$
\lim _{\ell \rightarrow \infty} \alpha^{k+1}\left(z_{\ell}, i\right)=\lim _{\ell \rightarrow \infty} \frac{\alpha^{k}\left(G\left(z_{\ell}, i\right)\right)-V\left(z_{\ell}, i\right)}{D G\left(z_{\ell}, i\right)} .
$$

Since the accumulation points of the sequence $G\left(z_{\ell}, i\right)$ belongs to $C(F)$ we have

$$
\lim _{\ell \rightarrow \infty} \alpha^{k}\left(G\left(z_{\ell}, i\right)\right)=0 .
$$

By Lemma 8.3 it follows that

$$
\lim _{\ell \rightarrow \infty} V\left(z_{\ell}, i\right)=0 .
$$

Since by the complex bounds we have that

$$
\inf _{(z, j) \in \mathscr{D}}|D G(z, j)|>0
$$

we conclude by (103) that

$$
\lim _{\ell \rightarrow \infty} \alpha^{k+1}\left(z_{\ell}, i\right)=0 .
$$

so $\alpha^{k+1}$ is continuous at points in $C(F)$. By the same argument in the end of the proof of Lemma 8.4 we conclude that $\alpha^{k+1}$ is a quasiconformal vector field on $\mathbb{C}_{n}$ and

$$
\sup _{\mathbb{C}_{n}}\left|\bar{\partial} \alpha^{k+1}\right| \leq \sup _{\mathbb{C}_{n}}\left|\bar{\partial} \alpha^{k}\right|
$$

Given a point $(z, j) \in \mathbb{C}_{n}$ such that there exists $k_{0} \geq 0$ such that $G^{k}(z, j) \in \mathscr{D}$ for every $k<k_{0}$ and $G^{k_{0}}(z, j) \notin \mathscr{D}$, we claim that $\alpha^{k}(z, j)=\alpha^{k_{0}}(z, j)$ for every $k \geq k_{0}$. Note that $\alpha^{k}(z, j)=\alpha^{0}(z, j)$ for every $(z, j) \notin \mathscr{D}$, so the claim holds for $k_{0}=0$. Suppose by induction on $k_{0}$ that the claim hold for $k_{0}$. If $G^{i}(z, j) \in \mathscr{D}$ for $i \leq k_{0}$ and $G^{k_{0}+1}(z, j) \notin \mathscr{D}$ then $G^{i}(G(z, j)) \in \mathscr{D}$ for $i<k_{0}$ and $G^{k_{0}}(G(z, j)) \notin \mathscr{D}$, so by the induction assumption $\alpha^{k}(G(z, j))=\alpha^{k_{0}}(G(z, j))$ for every $k \geq k_{0}$. Since $G(z, j) \in \mathscr{D}$ we have by (102) that

$$
\alpha^{k+1}(z, j)=\frac{\alpha^{k}(G(z, j))-V(z, j)}{D G(z, j)}=\frac{\alpha^{k_{0}}(G(z, j))-V(z, j)}{D G(z, j)}=\alpha^{k_{0}+1}(z, j),
$$

for every $k \geq k_{0}$, which proves the claim.

By Proposition 6.2 the sequence $\alpha^{k}$ converges almost everywhere. By (104) and McMullen [32] we have that every subsequence $\alpha^{k}$ has a subsequence that converges uniformly to some quasiconformal vector field. So $\alpha^{k}$ converges uniformly to a quasiconformal vector field $\alpha$. Taking $m \rightarrow \infty$ in (101) we conclude that

$$
V(z, j)=\alpha \circ G(z, j)-D G(z, j) \cdot \alpha(z, j),
$$


for every $(z, j) \in \mathscr{D}$ and in particular for every $(z, j) \in P(F) \backslash C(F)$. Since $\alpha(q)=0$ for every $q \in C(F)$, by Corollary 7.3 we conclude that $v \in E^{h}(F)$.

\section{Transversal families have hyperbolic parameters.}

From now on we will consider only real maps. In particular $\mathcal{B}_{\text {nor }}^{\mathbb{R}}(U)$ will now denote the real Banach space of all $F \in \mathcal{B}_{\text {nor }}(U)$ which are real on the real line. Since $\Omega_{n, p}$ is a hyperbolic set we can define the stable

$$
G \mapsto E_{G}^{h}
$$

and unstable

$$
G \mapsto E_{G}^{u}
$$

subspace distributions defined for $G \in \Omega_{n, p}$, and the corresponding projections on the spaces

and

$$
\pi_{G}^{h}: T \mathcal{B}_{n o r}^{\mathbb{R}}(U) \rightarrow E_{G}^{h}
$$

$$
\pi_{G}^{u}: T \mathcal{B}_{n o r}^{\mathbb{R}}(U) \rightarrow E_{G}^{u}
$$

Recall that $U=D_{\delta_{0}, \theta_{0}}$. As defined in [43] we also have the adapted norms $|\cdot|_{G, 0}$ that satisfy

$$
|v|_{G, 0}=\left|\pi_{G}^{h}(v)\right|_{G, 0}+\left|\pi_{G}^{u}(v)\right|_{G, 0},
$$

and the family of cones $C_{\epsilon}^{u}(G)$, with $\epsilon>0$, for which $v \in C_{\epsilon}^{u}(G)$ if and only if

$$
\left|\pi_{G}^{h}(v)\right|_{G, 0} \leq \epsilon\left|\pi_{G}^{u}(v)\right|_{G, 0} .
$$

Those cones are unstable and forward-invariant for the action of $\mathcal{R}$ on $\Omega_{n, p}$ provided $\epsilon$ is small enough. In particular if $\epsilon$ is small there is $\theta>1$ such that for every $F \in \mathcal{B}_{\text {nor }}^{\mathbb{R}}(U)$ close enough to some $G \in \Omega_{n, p}$, and $v \in C_{2 \epsilon}^{u}(G)$

$$
\left|D \mathcal{R}_{F} \cdot v\right|_{\mathcal{R} G, 0} \geq \theta|v|_{G, 0}
$$

and moreover there is $\epsilon^{\prime} \in(0, \epsilon)$ such that

$$
\left|\pi_{\mathcal{R} G}^{h}\left(D \mathcal{R}_{F} \cdot v\right)\right|_{\mathcal{R} G, 0} \leq 2 \epsilon^{\prime}\left|\pi_{\mathcal{R} G}^{u}\left(D \mathcal{R}_{F} \cdot v\right)\right|_{\mathcal{R} G, 0} .
$$

Furthermore there is $\lambda \in(0,1)$ such that for every $G \in \Omega_{n, p}, v \in E_{G}^{h}$ and $k \in \mathbb{N}$

$$
\left|D \mathcal{R}_{G}^{k} \cdot v\right|_{\mathcal{R}^{k} G, 0} \leq \lambda^{k}|v|_{G, 0} .
$$

Define the $\delta$-shadow of $G$ as

$$
W_{\delta}^{s}(G)=\left\{F \in \mathcal{W}: \operatorname{dist}_{\mathcal{B}_{\text {nor }}^{\mathbb{R}}(U)}\left(\mathcal{R}^{k} F, \mathcal{R}^{k} G\right) \leq \delta, \text { for every } k \geq 0\right\},
$$

and the $\delta$-shadow of $\Omega_{p, n}$ as

$$
W_{\delta}^{s}\left(\Omega_{p, n}\right)=\cup_{G \in \Omega_{p, n}} W_{\delta}^{s}(G) .
$$

We also define

$$
\mathbb{B}_{G}^{u}\left(v_{0}, \delta\right)=\left\{v \in E_{G}^{u} \cap \mathcal{B}^{\mathbb{R}}(U):\left|v-v_{0}\right|_{G, 0} \leq \delta\right\},
$$

where $v_{0} \in E_{G}^{u} \cap \mathcal{B}^{\mathbb{R}}(U)$, and

$$
E_{G}^{h}+G=\left\{v+G: v \in E_{G}^{h}\right\} .
$$

Let $\delta_{3}>0$ (we will use this notation to follow [43]). Define $\mathcal{T}_{0}^{1}(G, \delta, \epsilon$ ), with $\delta \in\left(0, \delta_{3}\right)$, as the set of $C^{1}$ functions

$$
\mathcal{H}: \mathbb{B}_{G}^{u}\left(v_{0}, \delta\right) \rightarrow E_{G}^{h}+G,
$$


with $v_{0} \in E_{G}^{u} \cap \mathcal{B}^{\mathbb{R}}(U)$, such that

$$
|D \mathcal{H}|_{(G, 0),(G, 0)} \leq \epsilon .
$$

and

$$
F_{0}=v_{0}+\mathcal{H}\left(v_{0}\right) \in W_{\delta_{3}}^{s}(G)
$$

We will call $F_{0}$ the base point of $\mathcal{H}$. In particular $w+D_{x} \mathcal{H} \cdot w \in C^{u}(G)$, for every $x \in \mathbb{B}_{G}^{u}\left(v_{0}, \delta\right), w \in E^{u}(G)$, and $G \in \Omega_{p, n}$.

Denote

$$
\hat{\mathcal{H}}=\left\{v+\mathcal{H}(v): v \in \mathbb{B}_{G}^{u}\left(v_{0}, \delta\right)\right\} .
$$

The Transversal Empty Interior assumption for the renormalization operator is the main result of this section.

Corollary 9.1 (Transversal Empty Interior Assumption). For every small $\epsilon>0$ we can choose $\delta_{3}$ small enough such that the following holds. For every $G \in \Omega_{p, n}$ and for every $C^{1+\text { Lip }}$ function $\mathcal{H} \in \mathcal{T}_{0}^{1}\left(G, \delta^{\prime}, \epsilon\right)$, with $\delta^{\prime}<\delta_{3}$, we have that $\hat{\mathcal{H}} \cap W_{\delta_{3}}^{s}(\Omega)$ has empty interior in $\hat{\mathcal{H}}$.

This property is closely related with the fact that maps $F$ that are infinitely renormalizable with bounded combinatorics can be approximated by hyperbolic maps.

We are going to introduce notation from [43]. Denote by $C^{\omega_{\mathbb{R}}}\left([-1,1]^{j}, \mathcal{B}_{\text {nor }}^{\mathbb{R}}(U)\right)$ the space of functions

$$
\gamma:(-1,1)^{j} \rightarrow \mathcal{B}_{\text {nor }}^{\mathbb{R}}(U)
$$

which can be extended to a complex analytic function

$$
\gamma: \mathbb{D}^{j} \rightarrow \mathcal{B}_{\text {nor }}(U),
$$

and moreover there is a continuous extension of $\gamma$ to $\overline{\mathbb{D}}^{j}$. Endowed with the sup norm on $\overline{\mathbb{D}}^{j}$ the space $C^{\omega_{\mathbb{R}}}\left([-1,1]^{j}, \mathcal{B}_{n o r}^{\mathbb{R}}(U)\right)$ is a real Banach space.

Endow $T_{\mathbb{C}}=\overline{\mathbb{D}}^{\mathbb{N}}$ with the product topology. Let $\Gamma^{\omega}\left(\mathcal{B}_{\text {nor }}(U)\right)$ be the set of continuous functions $\gamma: T_{\mathbb{C}} \mapsto \mathcal{B}_{\text {nor }}(U)$ which are holomorphic when we fix all but a finite number of entries of $\lambda \in T_{\mathbb{C}}$ and $\left|\lambda_{i}\right|<1$ for every $i$. Endowing $\Gamma^{\omega}\left(\mathcal{B}_{\text {nor }}(U)\right)$ with the sup norm we obtain a complex Banach space.

Note that since $U$ is symmetric with respect to the real line, that is, $(z, i) \in U$ iff $(\bar{z}, i) \in U$, there is a complex conjugation on the complex Banach space $\mathcal{B}(U)$ defined by $\bar{f}(z)=\overline{f(\bar{z})}$ for $f \in \mathcal{B}(U)$. Define $\Gamma^{\omega_{\mathbb{R}}}\left(\mathcal{B}_{\text {nor }}^{\mathbb{R}}(U)\right)$ as the real Banach space that consists of the restrictions to $T=[-1,1]^{\mathbb{N}}$ of functions $\gamma \in \Gamma^{\omega}\left(\mathcal{B}_{\text {nor }}(U)\right)$ satisfying $\gamma(\bar{\lambda})=\overline{\gamma(\lambda)}$.

We say that a set $\Theta \subset \mathcal{B}_{\text {nor }}(U)$ is a $\Gamma^{\omega_{\mathbb{R}}}\left(\mathcal{B}_{\text {nor }}^{\mathbb{R}}(U)\right)$-null set is there exists a residual subset $\mathcal{F} \subset \Gamma^{\omega_{\mathbb{R}}}\left(\mathcal{B}_{\text {nor }}^{\mathbb{R}}(U)\right)$ such that

$$
m\left(\lambda \in[-1,1]^{\mathbb{N}}: \gamma(\lambda) \in \Theta\right)=0
$$

for every $\gamma \in \mathcal{F}$. Here $m$ is the product measure obtained considering the normalised Lebesgue measure on each copy of $[-1,1]$.

The Transversal Empty Interior property will allows us to apply [43, Theorem $1]$ to the renormalization operator. Indeed we already verified that

- $\mathcal{R}: \mathcal{W}^{\mathbb{R}} \rightarrow \mathcal{B}_{\text {nor }}^{\mathbb{R}}(U)$ is a real analytic map. Here $\mathcal{W}^{\mathbb{R}}=\mathcal{W} \cap \mathcal{B}_{\text {nor }}^{\mathbb{R}}(U)$.

- The map $\mathcal{R}$ is a strongly compact operator (Remark 3.1),

- $\Omega_{n, p}$ is a hyperbolic set (Theorem 5), 
- For every $F \in \mathcal{R}^{-i} W_{\delta}^{s}\left(\Omega_{n, p}\right)$, with $i \in \mathbb{N}$ and $\delta>0$, we have that $D_{F} \mathcal{R}^{i}\left(T_{F} \mathcal{B}_{\text {nor }}^{\mathbb{R}}(U)\right)$ is dense in $T_{\mathcal{R}^{i} F} \mathcal{B}_{\text {nor }}^{\mathbb{R}}(U)$. This is an easy consequence of Theorem 3.

so [43, Theorem 1] in our setting boils down to

Theorem 6. ( $[43$, Theorem 1]) Suppose that the renormalization operator $\mathcal{R}$ satisfies additionally

A. There exists $\delta_{3}>0$ such that $W_{\delta_{3}}^{s}\left(\Omega_{n, p}\right)$ satisfies the Transversal Empty Interior assumption.

Then $W^{s}\left(\Omega_{n, p}\right)$ is a $\Gamma^{\omega_{\mathbb{R}}}\left(\mathcal{B}_{\text {nor }}^{\mathbb{R}}(U)\right)$-null set. Indeed for every $j$ there exists a residual set of real-analytic maps $\gamma \in C^{\omega_{\mathbb{R}}}\left([-1,1]^{j}, \mathcal{B}_{\text {nor }}^{\mathbb{R}}(U)\right)$ such that

$$
m\left(t \in[-1,1]^{j}: \gamma(t) \in W^{s}\left(\Omega_{n, p}\right)\right)=0 .
$$

Here $m$ is the Lebesgue measure on $[-1,1]^{j}$.

In particular this implies that a generic real-analytic finite-dimensional family in $\mathcal{W}^{\mathbb{R}}$ intersects $W^{s}\left(\Omega_{n, p}\right)$ on a subset with zero Lebesgue measure. So we have a version of Theorem A. for real-analytic families of extended maps that belong to $\mathcal{W}^{\mathbb{R}}$. Indeed the full-blown version of Theorem A. is proven in Section 10.

Proposition 9.2. For every $\epsilon>0$ small enough there is $\gamma>0$ with the following property. Suppose that $F \in \mathcal{B}_{\text {nor }}^{\mathbb{R}}(U)$, that $F$ has a polynomial-like extension $F: \hat{V}^{0} \rightarrow \hat{V}^{1}$, with $\bar{U} \subset \hat{V}^{0}$, and

$$
\operatorname{dist}_{\mathcal{B}_{\text {nor }}^{\mathbb{R}}(U)}(F, G)<\gamma,
$$

for some $G \in \Omega_{p, n}$. If

$$
v \in \hat{E}_{F}^{h} \cap C_{2 \epsilon}^{u}(G)
$$

and $v \in \mathcal{B}^{\mathbb{R}}\left(\hat{V}^{0}\right) \cap \mathcal{B}^{\mathbb{R}}(U)$ then $v=0$.

Proof. Suppose by contradiction that there exist sequences $G_{i} \in \Omega_{p, n}, F_{i} \in \mathcal{B}_{\text {nor }}^{\mathbb{R}}(U)$ and $v_{i} \in \mathcal{B}^{\mathbb{R}}\left(\hat{V}_{i}^{0}\right)$ such that

- We have

$$
\operatorname{dist}_{\mathcal{B}_{\text {nor }}^{\mathbb{R}}(U)}\left(F_{i}, G_{i}\right) \rightarrow_{i} 0
$$

- The maps $F_{i}$ have a polynomial-like of type $n$ extension $F_{i}: \hat{V}_{i}^{0} \rightarrow \hat{V}_{i}^{1}$ and $\bar{U} \subset \hat{V}_{i}^{0}$.

- The vectors satisfy $v_{i} \in \hat{E}_{F_{i}}^{h} \cap C_{2 \epsilon}^{u}\left(G_{i}\right),\left|v_{i}\right|_{\mathcal{B}^{\mathbb{R}}(U)} \neq 0$ and $v_{i} \in \mathcal{B}^{\mathbb{R}}\left(\hat{V}_{i}^{0}\right)$.

In particular for large $i$ the critical points of $F_{i}$ belongs to $K\left(F_{i}\right)$ and $F_{i}$ is renormalizable. Without loss of generality we can assume that $\left|v_{i}\right|_{\mathcal{B}(U)}=1$ for every $i$. Since $v_{i} \in \hat{E}^{h}\left(F_{i}\right)$ and $F_{i}$ are very close to $\Omega_{n, p}$ we have that $\mathcal{R} F_{i}$ has a polynomial-like extension of type $n$

$$
\mathcal{R} F_{i}: V_{i}^{0} \rightarrow V_{i}^{1}
$$

with $\bmod V_{i}^{1} \backslash V_{i}^{0}>\epsilon_{0}$. Moreover $D_{F_{i}} \mathcal{R} \cdot v_{i} \in \hat{E}_{\mathcal{R} F_{i}}^{h} \cap \mathcal{B}^{\mathbb{R}}\left(V_{i}^{0}\right)$ and there is $C>0$ such that

$$
\left|D_{F_{i}} \mathcal{R} \cdot v_{i}\right|_{\mathcal{B}^{\mathbb{R}}\left(V_{i}^{0}\right)} \leq C
$$

for every large $i$. Note that $\mathcal{R} F_{i}: V_{i}^{0} \rightarrow V_{i}^{1}$ is real on the real line and consequently it is hybrid conjugate with a real polynomial of type $n$ (see the Straightening lemma in [40, Proposition 4.1]). It follows from Shen [37] that $\mathcal{R} F_{i}$ does not have invariant line fields on its Julia set. So one can use the infinitesimal pullback 
argument to conclude that there exist quasiconformal vector fields $\alpha_{i}: \mathbb{C}_{n} \rightarrow \mathbb{C}$ with $\sup _{i}\left|\bar{\partial} \alpha_{i}\right|_{\infty}<\infty$ such that

$$
D_{F_{i}} \mathcal{R} \cdot v_{i}=\alpha_{i} \circ \mathcal{R} F_{i}-D\left(\mathcal{R} F_{i}\right) \cdot \alpha_{i}
$$

on a domain a little bit smaller than $V_{i}^{0}$, and in particular on $U=D_{\delta_{0}, \theta_{0}}$. By a compactness argument [32] the sequence $\alpha_{i}$ has a convergent subsequence that converges to a quasiconformal vector field $\alpha$. Let

$$
D_{\delta}=\{x \in \mathbb{C}: \operatorname{dist}(x,[-1,1])<\delta\} \times\{1, \ldots, n\} .
$$

Note that by (13) there exists $\delta^{\prime}>\delta_{0}$ such that $\bar{U} \subset D_{\delta^{\prime}} \subset V_{i}^{0}$ for every $i$. Since

$$
\left|D_{F_{i}} \mathcal{R} \cdot v_{i}\right|_{\mathcal{B}\left(D_{\delta^{\prime}}\right)} \leq C,
$$

there exists a subsequence of $D_{F_{i}} \mathcal{R} \cdot v_{i}$ that converges to some $v$ on $\mathcal{B}^{\mathbb{R}}(U)$ satisfying $|v|_{\mathcal{B}(U)} \leq C$. Since $\Omega_{p, n}$ is compact, without loss of generality we can assume that $\mathcal{R} G_{i}$ (and so $\mathcal{R} F_{i}$ ) converges to some $G \in \Omega_{p, n}$. By (106) we obtain

$$
v=\alpha \circ G-D G \cdot \alpha
$$

on the pos-critical set of $G$. Since there are not invariant line fields supported in the Julia set of $G$, by the infinitesimal pullback argument we conclude that $v \in E_{G}^{h}$. In particular

$$
\left|D \mathcal{R}_{G}^{k} \cdot v\right|_{\mathcal{R}^{k}(G), 0} \leq \lambda^{k}|v|_{G, 0} \leq C_{1} \lambda^{k} .
$$

On the other hand since $v_{i} \in C_{2 \epsilon}^{u}\left(G_{i}\right)$ and $\epsilon$ is small we have

$$
\left|D_{G_{i}} \mathcal{R} \cdot v_{i}\right|_{\mathcal{R}\left(G_{i}\right), 0} \geq \theta\left|v_{i}\right|_{G_{i}, 0} \geq C_{2} \theta\left|v_{i}\right|_{\mathcal{B}^{\mathbb{R}}(U)}=C_{2} \theta>0,
$$

The compactness of $\Omega_{p, n}$ gives $\lim _{i} D_{G_{i}} \mathcal{R} \cdot v_{i}=v$ and consequently for every $k \geq 1$

$$
\lim _{i} D \mathcal{R}_{G_{i}}^{k} \cdot v_{i}=D \mathcal{R}_{G}^{k-1} \cdot v
$$

and we have that $v_{i} \in C_{2 \epsilon}^{u}\left(G_{i}\right)$ so for $k \geq 1$

$$
\left|D \mathcal{R}_{G_{i}}^{k} \cdot v_{i}\right|_{\mathcal{R}^{k}\left(G_{i}\right), 0} \geq \theta^{k-1}\left|D \mathcal{R}_{G_{i}} \cdot v_{i}\right|_{\mathcal{R} G_{i}, 0} \geq C_{2} \theta^{k} .
$$

Taking the limit on $i$ we obtain

$$
\left|D \mathcal{R}_{G}^{k} \cdot v\right|_{\mathcal{R}^{k}(G), 0} \geq C_{2} \theta^{k} .
$$

Since $\lambda<1<\theta$ we conclude that (107) and (108) give us a contradiction.

Proposition 9.3. For $\epsilon>0$ small we can choose $\delta_{3}$ small enough such that for every $\delta^{\prime} \in\left(0, \delta_{3}\right)$ the following holds. Let $\mathcal{H}$ be a $C^{1+\text { Lip }}$ function

$$
\mathcal{H}: \mathbb{B}_{G}^{u}\left(u_{0}, \delta^{\prime}\right) \rightarrow E_{G}^{h}+G
$$

such that $\mathcal{H} \in \mathcal{T}_{0}^{1}\left(G, \delta^{\prime}, 2 \epsilon\right)$, where $G \in \Omega_{p, n}$. Then there exists $w \in \mathbb{B}_{G}^{u}\left(u_{0}, \delta^{\prime}\right)$ such that $w+\mathcal{H}(w)$ is a map whose critical points belong to the same periodic orbit.

Proof. Define

$$
\tilde{\mathcal{H}}: \mathbb{B}_{G}^{u}\left(u_{0}, \delta^{\prime}\right) \times\left\{v \in E^{h}(G):|v| \leq \delta^{\prime}\right\} \rightarrow \mathcal{B}_{\text {nor }}^{\mathbb{R}}(U)
$$

as $\tilde{\mathcal{H}}(u, v)=u+\mathcal{H}(u)+v$. Let $F=u_{0}+\mathcal{H}\left(u_{0}\right)$. Note that $\tilde{\mathcal{H}}$ is a homeomorphism on its image, which is an open neighbourhood of $F$. Define $\tilde{\mathcal{H}}_{1}(u, v)=\mathcal{R} \tilde{\mathcal{H}}(u, v)$. If $\delta_{3}$ is small enough there is a smooth family of domains $\hat{U}_{(u, v)}$ such that for every $(u, v)$ in the domain of $\tilde{\mathcal{H}}_{1}$ we have that

$$
\tilde{\mathcal{H}}_{1}(u, v): \hat{U}_{(u, v)} \rightarrow \hat{V}
$$


is a polynomial-like map of type $n$ such that

$$
\bmod \hat{V} \backslash \hat{U}_{(u, v)}>\epsilon_{0} .
$$

Reducing $\hat{V}$ a little bit, for every

$$
(w, z) \in E^{u}(G) \times E^{h}(G)
$$

we have

$$
D_{u} \tilde{\mathcal{H}}_{1}(u, v) \cdot w+D_{v} \tilde{\mathcal{H}}_{1}(u, v) \cdot z \in \mathcal{B}\left(\hat{U}_{(u, v)}\right)
$$

Moreover if $w \in E^{u}(G) \backslash\{0\}$ we have

$$
D_{u} \tilde{\mathcal{H}}_{1}(u, v) \cdot w \in C_{2 \epsilon}^{u}(\mathcal{R} G)
$$

so by Proposition 9.2,

$$
D_{u} \tilde{\mathcal{H}}_{1}(u, v) \cdot w \notin E_{\tilde{\mathcal{H}}_{1}(u, v)}^{h},
$$

and moreover for every $(w, z) \in E^{u}(G) \times E^{h}(G)$ such that

$$
D_{u} \tilde{\mathcal{H}}_{1}(u, v) \cdot w+D_{v} \tilde{\mathcal{H}}_{1}(u, v) \cdot z \in E_{\tilde{\mathcal{H}}_{1}(u, v)}^{h},
$$

we have

$$
D_{u} \tilde{\mathcal{H}}_{1}(u, v) \cdot w+D_{v} \tilde{\mathcal{H}}_{1}(u, v) \cdot z \notin C_{2 \epsilon}^{u}(\mathcal{R} G) .
$$

The image of $\tilde{\mathcal{H}}$ is an open neighbourhood of $u_{0}+\mathcal{H}\left(u_{0}\right) \in W_{\delta_{3}}^{s}(G)$, with $G \in$ $\Omega_{n, p}$, in particular by Proposition 4.7 there exists $\left(u_{1}, v_{1}\right)$ such that $\tilde{\mathcal{H}}\left(u_{1}, v_{1}\right)$ is a map whose critical points belong to the same periodic orbit, and consequently $\tilde{\mathcal{H}}_{1}\left(u_{1}, v_{1}\right)=\mathcal{R} \tilde{\mathcal{H}}\left(u_{1}, v_{1}\right)$ is also a map whose critical points belong to the same periodic orbit. Furthermore one can choose $\left(u_{1}, v_{1}\right)$ arbitrarily close to $\left(u_{0}, 0\right)$. If $v_{1}=0$ choose $w=u_{1}$ and we finished the proof in this case. Otherwise $v_{1} \neq 0$ and we consider the $C^{1+\text { Lip }}$ smooth map

$$
(u, t, x) \in E^{u}(G) \times \mathbb{R} \times U \mapsto f_{(u, t)}(x):=\tilde{\mathcal{H}}_{1}\left(u, t v_{1}\right)(x) .
$$

The critical points of $f_{\left(u_{1}, 1\right)}$ belong to the same periodic orbit, so there are natural numbers $i_{k}, k=1, \ldots, n$ and we can index the critical points

$$
\text { Crit }=\{(0, j)\}_{0 \leq j \leq n-1}=\left\{\left(0, j_{k}\right)\right\}_{0 \leq k \leq n-1}
$$

in such way that for every $k \leq n-1$

$$
f_{\left(u_{0}, 1\right)}^{i_{k}}\left(0, j_{k}\right)=\left(0, j_{k+1 \bmod n}\right) \text { and } f_{\left(u_{0}, 1\right)}^{i}\left(0, j_{k}\right) \notin \text { Crit for } i<i_{k} .
$$

We claim that there is a function $t \mapsto u(t)$, defined for every $t \in[0,1]$ such that

$$
f_{(u(t), t)}^{i_{k}}\left(0, j_{k}\right)=\left(0, j_{k+1 \bmod n}\right) \text { and } f_{(u(t), t)}^{i}\left(0, j_{k}\right) \notin \text { Crit for } i<i_{k},
$$

for every $k \leq n-1$. Indeed, let $Y$ be the set of $q \in[0,1]$ such that there exists a continuous function $u$ defined on $[q, 1]$ such that (111) holds for every $t \in[q, 1]$ and $u(1)=u_{1}$. Note that $1 \in Y$. We need to show that $0 \in Y$. It is enough to show that $Y$ is a open and closed subset of $[0,1]$. Indeed, suppose that $\left(u_{2}, t_{2}\right)$ satisfies

$$
f_{\left(u_{2}, t_{2}\right)}^{i_{k}}\left(0, j_{k}\right)=\left(0, j_{k+1 \bmod n}\right) \text { and } f_{\left(u_{2}, t_{2}\right)}^{i}\left(0, j_{k}\right) \notin \text { Crit for } i<i_{k},
$$

for every $k \leq n-1$. Note that the linear map

$$
w \mapsto\left(D_{u} f_{\left(u_{2}, t_{2}\right)}^{i_{k_{2}}}\left(0, j_{k}\right) \cdot w\right)_{0 \leq k \leq n-1}
$$

is invertible, otherwise it would exists $w \in E^{u}(G) \backslash\{0\}$ such that

$$
D_{u} f_{\left(u_{2}, t_{2}\right)}^{i_{k}}\left(0, j_{k}\right) \cdot w=0
$$


for every $k$, so using the infinitesimal pullback argument one can conclude that

$$
D_{u} \tilde{\mathcal{H}}_{1}\left(u_{2}, t_{2} v_{1}\right) \cdot w \in E_{\tilde{\mathcal{H}}_{1}\left(u_{2}, t_{2} v_{1}\right)}^{h},
$$

which contradicts (109). So by the Implicit Function Theorem there exists an open interval $O$ with $t_{2} \in O$ such that there is a unique continuous function $u$ defined on $O$ such that (111) holds for every $t \in O$ and $u\left(t_{2}\right)=u_{2}$. We conclude that $Y$ is an open set and that for each $q \in Y$ there exists an unique continuous function $U$ defined on $[q, 1]$ and satisfying and (111) and $u(1)=u_{1}$. To show that $Y$ is closed, suppose that $q_{n} \in Y$ is a decreasing sequence converging to some $q \in[0,1]$. Then there exists a unique continuous function $u$ defined in $(q, 1]$ such that $u(1)=u_{1}$ and (111) holds. We claim that $u$ is a Lipchitz function on $(q, 1]$, so we can extend it to a continuous function $u$ defined in $[q, 1]$. Indeed note that

$$
\partial_{t} f_{(u(t), t)} \in E_{f_{(u(t), t)}^{h}}^{h},
$$

so by $(110)$

$$
\partial_{t} f_{(u(t), t)} \notin C_{2 \epsilon}^{u}(\mathcal{R} G)
$$

Moreover

$$
\partial_{t} f_{(u(t), t)}=D \mathcal{R}_{u(t)+\mathcal{H}(u(t))+t v_{1}} \cdot\left(u^{\prime}(t)+D_{u} \mathcal{H}_{u(t)} \cdot u^{\prime}(t)+v_{1}\right)
$$

Let

Note that

$$
y=D \mathcal{R}_{u(t)+\mathcal{H}(u(t))+t v_{1}} \cdot\left(u^{\prime}(t)+D_{u} \mathcal{H}_{u(t)} \cdot u^{\prime}(t)\right) .
$$

$$
|y|_{\mathcal{R} G, 0} \geq \lambda \frac{1-2 \epsilon}{1+2 \epsilon}\left|u^{\prime}(t)\right|_{G, 0}
$$

Suppose that $\left|u^{\prime}(t)\right|_{G, 0} \geq L\left|v_{1}\right|_{G, 0}$. If $\delta^{\prime}$ is small enough then there is $C>0$ such that

$$
\left|\pi_{\mathcal{R} G, 0}^{u}\left(\partial_{t} f_{(u(t), t)}\right)\right|_{\mathcal{R} G, 0} \geq\left(1-\frac{C}{L \lambda} \frac{1+2 \epsilon}{1-2 \epsilon}\right)|y|_{\mathcal{R} G, 0}
$$

and

$$
\left|\pi_{\mathcal{R} G, 0}^{h}\left(\partial_{t} f_{(u(t), t)}\right)\right|_{\mathcal{R} G, 0} \leq\left(2 \epsilon^{\prime}+\frac{C}{L \lambda} \frac{1+2 \epsilon}{1-2 \epsilon}\right)|y|_{\mathcal{R} G, 0} .
$$

If $L$ is large enough then

$$
2 \epsilon^{\prime}+\frac{C}{L \lambda} \frac{1+2 \epsilon}{1-2 \epsilon} \leq 2 \epsilon\left(1-\frac{C}{L \lambda} \frac{1+2 \epsilon}{1-2 \epsilon}\right),
$$

which implies that $\partial_{t} f_{(u(t), t)} \in C_{2 \epsilon}^{u}(\mathcal{R} G)$. This contradicts (113). In particular there is $L$ satisfying $\left|u^{\prime}(t)\right|_{G, 0} \leq L\left|v_{1}\right|_{G, 0}$ for every $t \in(q, 1]$ and consequently $u$ is a Lipchitz function. So we can extend $u$ to a continuous map to $[q, 1]$. It is easy to see that (111) also holds for $t=q$. We conclude that $Y$ is closed. Since $Y$ is an open, closed, non empty subset of $[0,1]$ we conclude that $Y=[0,1]$, so in particular $0 \in Y$ and therefore there exists $w$ such that $f_{(w, 0)}=\tilde{\mathcal{H}}_{1}(w, 0)=\mathcal{R}(w+\mathcal{H}(w))$ is a map whose critical points belong to the same periodic orbit, and consequently $w+\mathcal{H}(w)$ has the same property.

Proof of Corollary 9.1. Let $\epsilon$ be small. It is easy to see that if $\delta_{3}>0$ is small enough then for every $G \in \Omega_{p, n}$ and for every $C^{1+\text { Lip }}$ function $\mathcal{H} \in \mathcal{T}_{0}^{1}\left(G, \delta^{\prime}, \epsilon\right)$, with $\delta^{\prime}<\delta_{3}$ and for every

$$
F \in \hat{\mathcal{H}} \cap W_{\delta_{3}}^{s}\left(\Omega_{n, p}\right),
$$


there is $G_{F} \in \Omega_{n, p}$ such that $F \in W_{\delta_{3}}^{s}\left(G_{F}\right)$ and $\delta^{\prime \prime}>0$ such that

$\left\{w+\mathcal{H}(w): w \in \mathbb{B}^{u}\left(v_{0}, \delta^{\prime}\right)\right\} \cap\left\{u+v+G_{F}: u \in \mathbb{B}_{G_{F}}^{u}\left(\pi_{G_{F}}^{u}\left(F-G_{F}\right), \delta^{\prime \prime}\right), v \in E_{G_{F}}^{h}\right\}$

is the graph $\hat{\mathcal{H}}_{F}$ of a $C^{1+L i p}$ function in $\mathcal{T}_{0}^{1}\left(G_{F}, \delta^{\prime \prime}, 2 \epsilon\right)$. By Proposition 9.3 there is $w \in \mathbb{B}_{G_{F}}^{u}\left(\pi_{G_{F}}^{u}\left(F-G_{F}\right), \delta^{\prime \prime}\right)$ such that $w+\mathcal{H}_{F}(w)$ is a map such that all its critical points belong to the same periodic orbit. In particular every map close enough to $w+\mathcal{H}_{F}(w)$ is a hyperbolic map with an attracting periodic orbit that attracts all its critical points. In particular we can find hyperbolic maps in $\hat{\mathcal{H}}$ arbitrarily close to $F$. Note that hyperbolic maps do not belong to $W_{\delta_{3}}^{s}\left(\Omega_{n, p}\right)$, since every map in $W_{\delta_{3}}^{s}\left(\Omega_{n, p}\right)$ is infinitely renormalizable.

\section{FAMilies OF MULTimOdAL MAPS}

In the beginning of Section 9 we saw that a version of Theorem A. for realanalytic families of extended maps that belong to $\mathcal{W}^{\mathbb{R}}$ can be obtained from the hyperbolicity of $\Omega_{n, p}$, the Empty Interior Transversality property and [43, Theorem 1]. This is not enough to our purposes, once Theorem A. deals with real-analytic families of multimodal maps. Indeed a multimodal map with more than a critical point is not an extended map.

To prove Theorem A. we will need a classic tool, inducing. We will associate to each real-analytic multimodal map $f$ that is close enough to an infinitely renormalizable multimodal map with bounded combinatorics a renormalization $F$ of $f$, that is an extended map in $\mathcal{W}^{\mathbb{R}}$. Indeed a renormalizable multimodal map can be renormalizable in many ways (it can have distinct cycles of restrictive intervals with disjoint orbits) and many times (it can have deeper and deeper renormalizations), so we need to mark $f$ with a restrictive interval $P$ in such way to make this association

$$
(f, P) \mapsto \mathcal{I}(f, P)=F
$$

well-behaved. Indeed we are going to see that $\mathcal{I}$ can be defined in such way that it is a real-analytic map defined in an open set of a real Banach space with image in $\mathcal{W}^{\mathbb{R}}$. The derivative $D_{(f, P)} \mathcal{I}$ of this map has dense image at every infinitely renormalizable marked multimodal maps $(f, P)$, which allows us to use Proposition 8.1 of [43] to conclude that $\mathcal{I}^{-1} W_{\delta}^{s}\left(\Omega_{n, p}\right)$ intersects a generic real-analytic family of multimodal maps on a set of parameters with zero Lebesgue measure. This is the main argument of the proof of Theorem A. We provide the complete proof below.

Let $V \subset \mathbb{C}$ be a connected open set, symmetric with respect to the real line $(z \in V$ implies $\bar{z} \in V)$ such that $[-1,1] \subset V$. In this section we will denote by $\mathcal{B}_{\mathbb{C}}$ by affine subspace of $\mathcal{B}(V)$ defined by the restrictions $f(-1)=f(-1)=-1$. Denote by $\mathcal{B}_{\mathbb{R}}$ the real Banach space of all functions $f \in \mathcal{B}_{\mathbb{C}}$ that are real on the $V \cap \mathbb{R}$.

Given $m \in \mathbb{N}$, let $\Gamma_{m}^{\omega_{\mathbb{R}}}\left(\mathcal{B}_{\mathbb{R}}\right)$ be the set of all continuous functions

$$
\gamma: \overline{\mathbb{D}}^{m} \rightarrow \mathcal{B}_{\mathbb{C}}
$$

that are complex analytic on $\mathbb{D}^{m}$ and such that $\gamma(\lambda) \in \mathcal{B}_{\mathbb{R}}$ for every $\lambda \in[-1,1]^{m}$. We can endow $\Gamma_{m}^{\omega_{\mathbb{R}}}\left(\mathcal{B}_{\mathbb{R}}\right)$ with the sup norm.

Let $\Gamma \subset \mathcal{B}_{\mathbb{R}}$ be the open subset of multimodal maps $f:[-1,1] \rightarrow[-1,1]$, where -1 is a repelling fixed point, $f^{\prime}(1) \neq 0$, with quadratic critical points, negative schwarzian derivative and $f(-1,1) \subset(-1,1)$. 
Denote by $\Gamma_{n} \subset \Gamma_{n}^{\omega_{\mathbb{R}}}\left(\mathcal{B}_{\mathbb{R}}\right)$ the subset of all families $\gamma$ such that $\gamma(\lambda) \in \Gamma$ for every $\lambda \in[-1,1]^{n}$. Note that $\Gamma_{n}$ is an open subset of $\Gamma_{n}^{\omega_{\mathbb{R}}}\left(\mathcal{B}_{\mathbb{R}}\right)$.

10.1. Generic families. Our main result for generic families is

Theorem 7 (Theorem A). For every $\gamma$ in a generic subset of $\Gamma_{m}$, the set $\Lambda$ of parameters $\lambda$ such that $\gamma(\lambda)$ has (at least) one solenoidal attractor with bounded combinatorics on $(-1,1)$ has zero Lebesgue measure.

Proof. We divide the proof in several steps.

Step I (Marking restrictive intervals). It turns out that a multimodal map may have many disjoint cycles of restrictive intervals. To deal with that we need to "mark" one of those restrictive intervals. To this end fix $j \in \mathbb{N}^{*}$ and $q, n \in \mathbb{N} \backslash\{0,1\}$. Let $\mathcal{O}_{j, q, n}$ be the set of all pairs $\left(f_{0}, P_{0}\right)$, such that

A. The map $f_{0} \in \Gamma$ has $j$ critical points in $[-1,1]$.

B. $P_{0}$ is a restrictive interval of $f_{0}$ such that each $f_{0}^{i}\left(P_{0}\right)$ have at most one critical point for every $i, \cup_{i} f_{0}^{i}\left(P_{0}\right)$ contains $n$ critical points and $P_{0}$ has a repelling periodic point in its boundary, with period $q^{\prime}<q$. In particular $f_{0}^{q^{\prime}}\left(\partial P_{0}\right) \subset \partial P_{0}$.

C. The $f_{0}$-forward orbit of any critical point on the orbit of such restrictive interval $P_{0}$ does not fall in the orbit of such periodic point.

Note that the image $\pi_{1}\left(\mathcal{O}_{j, q, n}\right)$ of the projection onto the first coordinate in $\mathcal{O}_{j, q, n}$ is an open subset of $\Gamma$. Of course the countable family

$$
\left\{\pi_{1}\left(\mathcal{O}_{j, q, n}\right)\right\}_{j, q, n}
$$

covers all infinitely renormalizable multimodal maps.

Fix $\left(f_{0}, P_{0}\right) \in \mathcal{O}_{j, q, n}$. By the implicit function theorem the repelling periodic point of $f_{0}$ in the boundary of $P_{0}$ has an analytic continuation that is also repelling and it defines a restrictive interval $P_{g}$ for each map $g$ in an open connected neighborhood $\mathcal{V}_{0}$ of $f_{0}$ on $\Gamma$ and such restrictive interval also satisfies properties A., B. and $\mathrm{C}$. In particular the family $\mathcal{F}$ of pairs $(\mathcal{V}, P)$ where

1. $\mathcal{V}$ is an open and connected subset of $\Gamma$, with $f_{0} \in \mathcal{V}$.

2. The real analytic function

$$
g \in \mathcal{V} \mapsto P(g)
$$

associate with each map $g \in \mathcal{V}$ a restrictive interval $P(g)$ of $g$ satisfying $(g, P(g)) \in \mathcal{O}_{j, q, n}$ and moreover $P\left(f_{0}\right)=P_{0}$.

is non empty and consequently by Zorn's Lemma $\mathcal{F}$ has a maximal element with respect to the order $\left(\mathcal{V}_{1}, P_{1}\right)<\left(\mathcal{V}_{2}, P_{2}\right)$ if and only if $\mathcal{V}_{1} \subset \mathcal{V}_{2}$ and $P_{2}(g)=P_{1}(g)$ for every $g \in \mathcal{V}_{1}$. We claim that such maximal element is unique.

We claim that if $\left(\mathcal{V}_{0}, P_{0}\right),\left(\mathcal{V}_{1}, P_{1}\right) \in \mathcal{F}$ then $P_{0}=P_{1}$ on $\mathcal{V}_{0} \cap \mathcal{V}_{1}$. Indeed since $f \in \mathcal{V}_{i}, i=0,1$, has always $j$ critical points (moving continously with respect to $f$, since they are quadratic) and a point $b_{f, i} \in \partial P_{i}(f)$ is a repelling periodic point of $f$ that is analytic continuation of $b_{f_{0}, 0}=b_{f_{0}, 1}$, it follows that all those periodic points have exactly the same combinatorics with respect to the symbolic dynamics defined by partition induced by the critical points. In particular if $f \in \mathcal{V}_{0} \cap \mathcal{V}_{1}$ then $b_{f, 0}, b_{f, 1}$ are repelling periodic points of $f$ with the same combinatorics. Since $f$ has negative schwarzian derivative, the minimal principle implies that $b_{f, 0}=b_{f, 1}$. This proves the claim. 
In particular the maximal element of $\mathcal{F}$, denoted by $\left(\mathcal{V}_{f_{0}, P_{0}}, P_{f_{0}, P_{0}}\right)$, can be described by

$$
\mathcal{V}_{f_{0}, P_{0}}=\cup_{(\mathcal{V}, P) \in \mathcal{F}} \mathcal{V}
$$

and $P_{f_{0}, P_{0}}(f)=P(f)$ for every $f \in \mathcal{V}$ satisfying $(\mathcal{V}, P) \in \mathcal{F}$. Note that

$$
\mathcal{G}=\left\{\mathcal{V}_{f_{0}, P_{0}}:\left(f_{0}, P_{0}\right) \in \mathcal{O}_{j, q, n}\right\}
$$

is a partition of $\mathcal{O}_{j, q, n}$. We claim that such partition has a countable number of elements. Indeed suppose that

$$
\left\{\left(f_{\lambda}, P_{\lambda}\right)\right\}_{\lambda \in \Lambda}
$$

is an uncountable family such that

$$
\mathcal{V}_{f_{\lambda}, P_{\lambda}} \neq \mathcal{V}_{f_{\mu}, P_{\mu}}
$$

for every $\lambda \neq \mu$. Choose a complex neighborhood $W$ of $[-1,1]$ such that $\bar{W} \subset U$. Then there exists a sequence $\lambda_{k} \in \Lambda, k \in \mathbb{N}$, such that

P1. There are $n$ and $q^{\prime}<q$ such that $\left(f_{\lambda_{k}}, P_{\lambda_{k}}\right)$ satisfies the conditions $A$ and $B$ for every $k$.

P2. $\lim _{k}\left(f_{\lambda_{k}}, P_{\lambda_{k}}\right)=\left(f_{\infty}, P_{\infty}\right)$ on $\mathcal{B}_{\mathbb{R}}(W)$, where $\left(f_{\infty}, P_{\infty}\right)$ is a multimodal map with $j$ quadratic critical points, negative schwarzian derivative and $f_{\infty}(-1,1) \subset(-1,1)$, and that also satisfies $A$., $B$. and $C$. for the very same $n$ and $q^{\prime}$ as in P1.

P3. There is $\theta>1$ such that if $b_{\lambda_{k}}$ is the repelling periodic point in the boundary of $P_{\lambda_{k}}$ then $\left|D f_{\lambda_{k}}^{q^{\prime}}\left(b_{\lambda_{k}}\right)\right|>\theta$ for every $k$.

P4. If $k \neq k^{\prime}$ then $\lambda_{k} \neq \lambda_{k^{\prime}}$.

By the implicit function theorem there is a ball $Y$ of $\mathcal{B}_{\mathbb{R}}(W)$ around $f_{\infty}$ and a real-analytic function $P$ defined in $Y$ such that for every $f \in Y$ we have that $P(f)$ is a restrictive interval for $f$ satisfying $A$ and $B$, and additionally $P\left(f_{\lambda_{k}}\right)=P_{\lambda_{k}}$ for every large $k$. In particular, choose $k_{0}, k_{1}$ large enough and a small connected open subset $\tilde{W} \subset \Gamma$ around the segment $\left\{t f_{\lambda_{k_{0}}}+(1-t) f_{\lambda_{k_{1}}}, t \in[0,1]\right\}$. Then the function $P$ is defined in $\tilde{W}$, which implies that

$$
\mathcal{V}_{f_{\lambda_{k_{0}}}, P_{\lambda_{k_{0}}}}=\mathcal{V}_{f_{\lambda_{k_{1}}}, P_{\lambda_{k_{1}}}},
$$

which is a contradiction. This completes the proof of our claim.

Step II. (Replacing multimodal maps by extended maps of type $n$ ) A real analytic multimodal map does not have the nice structure of a multimodal map of type $n$. Fix some open set $\mathcal{V}_{f_{0}, P_{0}} \subset \mathcal{O}_{j, q, n}$. We will replace every $g \in \pi_{1}\left(\mathcal{V}_{f_{0}, P_{0}}\right)$ by a induced map that is an extended map of type n. Denote by $I_{g}$ the extended map of type $n$ that is the renormalization of $g$ associated with the restrictive interval $P_{f_{0}, P_{0}}(g)$. Of course $I_{g}$ is a real-analytic extended map with negative schwarzian derivative and quadratic critical points.

Step III. (Compexification). Fix $p \geq 2$ Let $\mathcal{W} \subset \mathcal{B}_{\text {nor }}(U)$ be the domain of the complexification of the $p$-bounded renormalization operator $\mathcal{R}$ as defined Section 3. Note that if $I_{g}$ is infinitely renormalizable with $p$-bounded combinatorics we don't necessarily have that $I_{g} \in \mathcal{W}$. It may be the case that $I_{g}$ is not defined on the domain $U$, for instance. However by the beau complex bounds given by Proposition 2 and the universality result in Proposition 4.6 there is $k$ such that 
$R^{k^{\prime}}\left(I_{g}\right) \in \mathcal{W}$ for every $k^{\prime} \geq k$. Again by the beau complex bounds and Proposition 4.6 we can find open subsets $\mathcal{V}_{f_{0}, P_{0}}^{k} \subset \mathcal{V}_{f_{0}, P_{0}}$ such that for

E1. For every $g \in \mathcal{V}_{f_{0}, P_{0}}^{k}$ we have that $\mathcal{I}_{k}(g)=R^{k}\left(I_{g}\right)$ is well defined and it belongs to $\mathcal{W}$.

E2. For every $g \in \mathcal{V}_{f_{0}, P_{0}}^{k}$ that is infinitely renormalizable with $p$-bounded combinatorics we have $R^{k^{\prime}}\left(I_{g}\right) \in \mathcal{W}$ for every $k^{\prime} \geq k$.

E3. We have that

$$
\cup_{k} \mathcal{V}_{f_{0}, P_{0}}^{k}
$$

contains all infinitely maps in $\mathcal{V}_{f_{0}, P_{0}}$ which are renormalizable with $p$ bounded combinatorics.

The operator $\mathcal{I}_{k}$ has a complexification (it can the proven using exactly the same argument as in the complexification of the renormalization operator in Section 3). From now on we restrict $\mathcal{I}_{k}$ to real maps. Note that the image of the operator $D_{g} \mathcal{I}_{k}$ is dense in $T \mathcal{B}_{\text {nor }}^{\mathbb{R}}(U)$ (again, the argument is the same as with the renormalization operator in Theorem 3).

Step IV. (Applying the hyperbolicity of $\Omega_{n, p}$ ). Due Theorem 5 we have that $\Omega_{n, p}$ is a hyperbolic invariant set of $\mathcal{R}$. Moreover Corollary 9.1 says that $W^{s}\left(\Omega_{n, p}\right)$ has transversal empty interior. Theorem 3 tells us that $D_{F} \mathcal{R}\left(T_{F} \mathcal{B}_{\text {nor }}^{\mathbb{R}}(U)\right)$ is dense in $T_{\mathcal{R} F} \mathcal{B}_{\text {nor }}^{\mathbb{R}}(U)$ for every $F \in \mathcal{W}^{\mathbb{R}}$. So we conclude that $\mathcal{R}$ (restricted to real maps) satisfies the assumptions of Theorem 1 in [43] (taking $k=\omega_{\mathbb{R}}$ there). Now we can apply Proposition 8.1 of [43] taking $\mathcal{M}=\mathcal{I}_{k}$ to conclude that for a generic $\gamma \in \Gamma_{n}^{\omega_{\mathbb{R}}}\left(\mathcal{B}_{\mathbb{R}}\right)$ the set of parameters $\lambda \in[-1,1]^{n}$ where $\mathcal{I}_{k}(\gamma(\lambda))$ is infinitely renormalizable with $p$-bounded combinatorics has zero Lebesgue measure. Since there is just a countable number of choices for $k$, elements of $\mathcal{G}, q, p$ and $j$, we concluded the proof.

Indeed Proposition 8.1 of [43] implies an analogous result for finitely differentiable families of maps in $\Gamma$. We refer the reader to [43] for additional statements and definitions for this setting.

10.2. Transversal families of polynomial-like maps. Recall the definition of $\hat{E}_{f}^{h}$ and $\hat{E}_{f}^{v}$ in Section 4.2.

Theorem 8 (Transversal families). Let $\Lambda$ be an open subset of $\mathbb{C}^{d}$. Let

$$
\lambda \in \Lambda \mapsto f_{\lambda}: V_{\lambda}^{1} \rightarrow V_{\lambda}^{2}
$$

be a complex analytic family of polynomial-like maps such that for every $\lambda \in \Lambda \cap \mathbb{R}^{d}$ we have that $V_{\lambda}^{1}, V_{\lambda}^{2}$ are symmetric with respect with $\mathbb{R}$, its real trace is an interval, $f_{\lambda}(x) \in \mathbb{R}$ for every $x \in \mathbb{R}, f_{\lambda}$ has negative Schwarzian derivative and just quadratic critical points on the real line. Suppose that for every $\lambda_{0} \in \mathbb{R}^{d}$ such that $f_{\lambda_{0}}$ is infinitely renormalizable with bounded combinatorics we have that

(Transversality assumption.) Every holomorphic vector in a neighborhood of $K\left(f_{\lambda_{0}}\right)$ can be written as a sum of a vector in $\hat{E}_{f_{\lambda_{0}}}^{h}$ and a vector in

$$
\left.D_{\lambda} f_{\lambda}\right|_{\lambda=\lambda_{0}}\left(\mathbb{R}^{d}\right)
$$


Then the set of parameters $\lambda \in \Lambda \cap \mathbb{R}^{d}$ where $f_{\lambda}$ is infinitely renormalizable with bounded combinatorics has zero d-dimensional Lebesgue measure.

Proof. We can find a countable family of domains $U_{i} \subset \mathbb{C}$, symmetric with respect to $\mathbb{R}$, and open subsets $\Lambda_{i} \subset \Lambda \cap \mathbb{R}^{d}$ such that $\cup_{i} \Lambda_{i}=\Lambda \cap \mathbb{R}^{d}, f_{\lambda} \in \mathcal{B}\left(U_{i}\right)$, for $\lambda \in \Lambda_{i}$, with $K\left(f_{\lambda}\right) \cap \mathbb{R} \subset U_{i} \cap \mathbb{R}$, and

$$
\lambda \in \Lambda_{i} \mapsto f_{\lambda} \in \mathcal{B}\left(U_{i}\right)
$$

is an real analytic family. It is enough to prove the conclusion of Theorem 8 for each one of those families. So fix $i$. The proof goes as the proof of Theorem 7 . We can define the sets $\mathcal{O}_{j, q, n}$ replacing the pairs $(f, P)$ by pairs of the form $(\lambda, P)$, where $\lambda \in \Lambda_{i}$ and $P$ is a restrictive interval of $f_{\lambda}$. In a similar way we can define $\left(\mathcal{V}_{\lambda_{0}, P_{\lambda_{0}}}, P_{\lambda_{0}, P_{\lambda_{0}}}\right)$, the sets $\mathcal{V}_{\lambda_{0}, P_{\lambda_{0}}}^{k} \subset \Lambda_{i}$ and the real-analytic parametrized families

$$
\lambda \in \mathcal{V}_{\lambda_{0}, P_{\lambda_{0}}}^{k} \rightarrow \mathcal{I}_{k}\left(f_{\lambda}\right) \in \mathcal{W}
$$

Suppose that $f_{\lambda}$ is infinitely renormalizable with $p$-bounded combinatorics. Then

$$
D_{f_{\lambda}} \mathcal{I}_{k}\left(E_{f_{\lambda}}^{h}\right) \subset E_{\mathcal{I}_{k}\left(f_{\lambda}\right)}^{h} .
$$

This follows exactly as the proof of Proposition 4.2. On the other hand we know (see the proof of Theorem 7) that $\operatorname{Im} D_{f_{\lambda}} \mathcal{I}_{k}$ is dense in $T_{\mathcal{I}_{k}\left(f_{\lambda}\right)} \mathcal{B}_{\text {nor }}^{\mathbb{R}}(U)$. By the Transversality assumption this implies that there is a subspace $S_{\lambda} \subset \mathbb{R}^{d}$, with $\operatorname{dim} S_{\lambda}=n$, such that

$$
D_{f_{\lambda}} \mathcal{I}_{k} \cdot D_{\lambda} f_{\lambda}\left(S_{\lambda}\right) \pitchfork E_{\mathcal{I}_{k}\left(f_{\lambda}\right)}^{h} .
$$

Suppose that $\lambda_{0}$ is such that $f_{\lambda_{0}}$ is infinitely renormalizable with $p$-bounded combinatorics. Let $v_{1}, \ldots, v_{n}, v_{n+1}, \ldots, v_{d}$ be a basis of $\mathbb{R}^{d}$ such that $v_{1}, \ldots, v_{n}$ is a basis for $S_{\lambda_{0}}$. Then for every $\gamma=\left(\gamma_{1}, \ldots, \gamma_{d-n}\right) \in \mathbb{R}^{n-d}$ that is small enough we have that the family

$$
\theta=\left(\theta_{1}, \ldots, \theta_{n}\right) \mapsto g_{\theta}=f_{\lambda_{0}+\sum_{i=1}^{n} \theta_{i} v_{i}+\sum_{i=n+1}^{d} \gamma_{i-n} v_{i}},
$$

where $\theta$ is also small, satisfies

$$
D_{g_{\theta}} \mathcal{I}_{k} \cdot D_{\theta} g_{\theta}\left(\mathbb{R}^{n}\right) \pitchfork E_{\mathcal{I}_{k}\left(g_{\theta}\right)}^{h} .
$$

So by [43, Corollary 10.2] we have that for every small $\gamma$, the set of small parameters $\theta$ such that $g_{\theta}$ is infinitely renormalizable with bounded combinatorics has zero $n$-dimensional Lebesgue measure. By the Fubini's Theorem it follows that in a small neighborhood the parameter $\lambda_{0}$ the set of parameters $\lambda$ such that $f_{\lambda}$ is infinitely renormalizable with $p$-bounded combinatorics has zero $d$-dimensional Lebesgue measure. This completes the proof.

Proof of Theorem C. Let $f_{\lambda_{1}, \lambda_{2}}(z)=z^{3}-3 \lambda_{1}^{2} z+\lambda_{2}$. Note that if $\lambda_{1}=0$ then $f_{\lambda_{1}, \lambda_{2}}$ is not infinitely renormalizable, so we assume that $\lambda_{1} \neq 0$. Let $\lambda_{0}=(a, b)$, $a \neq 0$. Then

$$
\left.\partial_{\lambda} f_{\lambda}\right|_{\lambda=\lambda_{0}}\left(\mathbb{R}^{2}\right)=\{c z+d, c, d \in \mathbb{R}\}
$$

By Proposition 4.8 we have that $\hat{E}_{f_{\lambda}}^{v}$ is the space of cubic polynomials, so $\operatorname{dim} \hat{E}_{f_{\lambda}}^{v}=$ 4 and

We claim that

$$
\left.\partial_{\lambda} f_{\lambda}\right|_{\lambda=\lambda_{0}}\left(\mathbb{R}^{2}\right) \subset \hat{E}_{f_{\lambda}}^{v}
$$

$$
\left\{2 z^{3}-b,-3 z^{2}-3 a^{2}-1\right\} \subset \hat{E}_{f_{\lambda}}^{h} \cap \hat{E}_{f_{\lambda}}^{v}
$$


Indeed, let $H_{t}(z)=z / t$. Then

$$
2 z^{3}-b=\left.\partial_{t}\left(H_{t} \circ f_{a, b} \circ H_{t}^{-1}(z)\right)\right|_{t=1},
$$

so $2 z^{3}-b=\alpha_{1}\left(f_{a, b}(z)\right)-D f_{a, b}(z) \alpha_{1}(z)$, where $\alpha_{1}(z)=\left.\partial_{t} H_{t}(z)\right|_{t=1}=-z$. Let $S_{t}(z)=z-t$. Then

$$
3 z^{2}-3 a^{2}-1=\left.\partial_{t}\left(S_{t} \circ f_{a, b} \circ S_{t}^{-1}(z)\right)\right|_{t=0},
$$

so $3 z^{2}-3 a^{2}-1=\alpha_{2}\left(f_{a, b}(z)\right)-D f_{a, b}(z) \alpha_{2}(z)$, where $\alpha_{2}(z)=\left.\partial_{t} S_{t}(z)\right|_{t=0}=-1$. Since $\left\{1, z, 2 z^{3}-b, 3 z^{2}-3 a^{2}-1\right\}$ is a basis of $\hat{E}_{f_{\lambda}}^{v}$, by (27) and (117) we have that every holomorphic vector in a neighborhood of $K\left(f_{a, b}\right)$ can be written as a sum of a vector in $\hat{E}_{f_{a, b}}^{h}$ and a vector in $\left.\partial_{\lambda} f_{\lambda}\right|_{\lambda=\lambda_{0}}\left(\mathbb{R}^{2}\right)$. Now we apply Theorem 8.

10.3. Compositions of quadratic maps. We can say something about a specific family of extended maps of type $n$. This family was introduced in [40]. Let $\lambda=$ $\left(\lambda_{i}\right)_{i \leq n}$, with $\lambda_{i} \in[0,1]$ and define

$$
F_{\lambda}: \mathbb{C} \times\{i\}_{i \leq n} \rightarrow \mathbb{C} \times\{i\}_{i \leq n}
$$

as $F_{\lambda}(z, i)=\left(-2 \lambda_{i} z^{2}+2 \lambda_{i}-1, i+1 \bmod n\right)$.

It follows from the study in [40] that each possible combinatorial type of an infinitely renormalizable extended map of type $n$ with combinatorics bounded by $p$ can be realized by a unique parameter in $[0,1]^{n}$ and the set of such parameters $\Lambda_{p, n} \subset[0,1]^{n}$ is a Cantor set [40, Theorem 2]. The following result answers a conjecture in [40].

Theorem 9. We have that $m\left(\Lambda_{p, n}\right)=0$, where $m$ is the $n$-dimensional Lebesgue measure.

Proof. Due Corollary 10.2 in [43], it is enough to show that this family is transversal to the horizontal distribution $F \rightarrow E_{F}^{h}$. We will give a proof similar to the proof of the transversality of the quadratic family by Lyubich [27]. Indeed, suppose by contradiction that there exists $\lambda_{0} \in \Lambda_{p, n}$ and $w \in \mathbb{R}^{n} \backslash\{0\}$ such that

$$
v=\left.\partial_{\lambda} F_{\lambda}\right|_{\lambda=\lambda_{0}} \cdot w \in E_{F_{\lambda_{0}}}^{h} .
$$

So there is a quasiconformal vector field $\alpha_{0}$, defined in a neighborhood of the postcritical set $P\left(F_{\lambda_{0}}\right)$ satisfying (17) on $P\left(F_{\lambda_{0}}\right)$. Since this is a family of polynomials, the conformal dynamics outside the Julia set of $F_{\lambda_{0}}$ is always the same, so $v$ is also a vertical direction, that is, there exists a conformal vector field $\alpha_{1}$ defined outside the Julia set such that (17) holds outside the Julia set. Using the infinitesimal pullback argument we can find a quasiconformal vector field solution $\alpha$ that satisfies (17) everywhere and moreover it is conformal outside the Julia set. Since $F_{\lambda_{0}}$ does not support invariant line fields on its Julia set we conclude that $\alpha$ is conformal everywhere and indeed it is equal to zero, since it is zero at three points of $\overline{\mathbb{C}} \times\{i\}$, for each $i \leq n$. So $v=0$, which implies $w=0$.

Remark 10.1. Note that $\Lambda_{p, n}$ only includes the parameters where each renormalization involves all $n$ critical points, that is, each cycle of intervals covers all critical points. If we consider infinitely renormalizable maps where fewer points are involved then the set of parameters it is not a Cantor set anymore. However it is likely that this larger subset of parameters also have zero Lebesgue measure. 


\section{REFERENCES}

[1] L. V. Ahlfors. Lectures on quasiconformal mappings, volume 38 of University Lecture Series. American Mathematical Society, Providence, RI, second edition, 2006. With supplemental chapters by C. J. Earle, I. Kra, M. Shishikura and J. H. Hubbard.

[2] A. Avila and M. Lyubich. The full renormalization horseshoe for unimodal maps of higher degree: exponential contraction along hybrid classes. Publ. Math. Inst. Hautes Études Sci., (114):171-223, 2011.

[3] A. Avila, M. Lyubich, and W. de Melo. Regular or stochastic dynamics in real analytic families of unimodal maps. Invent. Math., 154(3):451-550, 2003.

[4] A. Avila, M. Lyubich, and W. Shen. Parapuzzle of the Multibrot set and typical dynamics of unimodal maps. J. Eur. Math. Soc. (JEMS), 13(1):27-56, 2011.

[5] A. Avila and C. G. Moreira. Phase-parameter relation and sharp statistical properties for general families of unimodal maps. In Geometry and dynamics, volume 389 of Contemp. Math., pages 1-42. Amer. Math. Soc., Providence, RI, 2005.

[6] A. M. Blokh and M. Y. Lyubich. Attractors of the transformations of the interval. Funktsional. Anal. i Prilozhen., 21(2):70-71, 1987.

[7] A. M. Blokh and M. Y. Lyubich. Decomposition of one-dimensional dynamical systems into ergodic components. The case of a negative Schwarzian derivative. Algebra i Analiz, 1(1):128145,1989

[8] H. Bruin, G. Keller, T. Nowicki, and S. van Strien. Wild Cantor attractors exist. Ann. of Math. (2), 143(1):97-130, 1996.

[9] H. Bruin, G. Keller, and M. St. Pierre. Adding machines and wild attractors. Ergodic Theory Dynam. Systems, 17(6):1267-1287, 1997.

[10] H. Bruin, W. Shen, and S. van Strien. Existence of unique SRB-measures is typical for real unicritical polynomial families. Ann. Sci. École Norm. Sup. (4), 39(3):381-414, 2006.

[11] T. Clark. Regular or stochastic dynamics in families of higher-degree unimodal maps. Ergodic Theory Dynam. Systems, 34(5):1538-1566, 2014.

[12] E. de Faria, W. de Melo, and A. Pinto. Global hyperbolicity of renormalization for $C^{r}$ unimodal mappings. Ann. of Math. (2), 164(3):731-824, 2006.

[13] W. de Melo and S. van Strien. One-dimensional dynamics, volume 25 of Ergebnisse der Mathematik und ihrer Grenzgebiete (3) [Results in Mathematics and Related Areas (3)]. Springer-Verlag, Berlin, 1993.

[14] B. Derrida, A. Gervois, and Y. Pomeau. Universal metric properties of bifurcations of endomorphisms. J. Phys. A, 12(3):269-296, 1979.

[15] M. J. Feigenbaum. Quantitative universality for a class of nonlinear transformations. J. Statist. Phys., 19(1):25-52, 1978.

[16] M. J. Feigenbaum. The universal metric properties of nonlinear transformations. J. Statist. Phys., 21(6):669-706, 1979.

[17] A. I. Gol'berg, Y. G. Sinaı̆, and K. M. Khanin. Universal properties of sequences of periodtripling bifurcations. Uspekhi Mat. Nauk, 38(1(229)):159-160, 1983.

[18] J. Graczyk and G. Światek. Generic hyperbolicity in the logistic family. Ann. of Math. (2), 146(1):1-52, 1997.

[19] J. Hu. Renormalization, rigidity, and universality in bifurcation theory. ProQuest LLC, Ann Arbor, MI, 1995. Thesis (Ph.D.)-City University of New York.

[20] M. V. Jakobson. Absolutely continuous invariant measures for one-parameter families of onedimensional maps. Comm. Math. Phys., 81(1):39-88, 1981.

[21] M. Kapovich. On the dynamics of pseudo-Anosov homeomorphisms on representation varieties of surface groups. Ann. Acad. Sci. Fenn. Math., 23(1):83-100, 1998.

[22] G. Keller and T. Nowicki. Fibonacci maps re(al)visited. Ergodic Theory Dynam. Systems, 15(1):99-120, 1995.

[23] O. Kozlovski, W. Shen, and S. van Strien. Density of hyperbolicity in dimension one. Ann. of Math. (2), 166(1):145-182, 2007.

[24] O. Kozlovski, W. Shen, and S. van Strien. Rigidity for real polynomials. Ann. of Math. (2), 165(3):749-841, 2007.

[25] O. E. Lanford, III. A computer-assisted proof of the Feigenbaum conjectures. Bull. Amer. Math. Soc. (N.S.), 6(3):427-434, 1982. 
[26] M. Lyubich. Dynamics of quadratic polynomials. I, II. Acta Math., 178(2):185-247, 247-297, 1997.

[27] M. Lyubich. Feigenbaum-Coullet-Tresser universality and Milnor's hairiness conjecture. Ann. of Math. (2), 149(2):319-420, 1999.

[28] M. Lyubich. Dynamics of quadratic polynomials. III. Parapuzzle and SBR measures. Astérisque, (261):xii-xiii, 173-200, 2000. Géométrie complexe et systèmes dynamiques (Orsay, 1995).

[29] M. Lyubich. Almost every real quadratic map is either regular or stochastic. Ann. of Math. (2), 156(1):1-78, 2002.

[30] R. S. MacKay and J. B. J. van Zeijts. Period doubling for bimodal maps: a horseshoe for a renormalisation operator. Nonlinearity, 1(1):253-277, 1988.

[31] C. T. McMullen. Complex dynamics and renormalization, volume 135 of Annals of Mathematics Studies. Princeton University Press, Princeton, NJ, 1994.

[32] C. T. McMullen. Renormalization and 3-manifolds which fiber over the circle, volume 142 of Annals of Mathematics Studies. Princeton University Press, Princeton, NJ, 1996.

[33] J. Milnor. On the concept of attractor. Comm. Math. Phys., 99(2):177-195, 1985.

[34] J. Milnor. Dynamics in one complex variable, volume 160 of Annals of Mathematics Studies. Princeton University Press, Princeton, NJ, third edition, 2006.

[35] R. J. Sacker and G. R. Sell. Existence of dichotomies and invariant splittings for linear differential systems. I. J. Differential Equations, 15:429-458, 1974.

[36] R. J. Sacker and G. R. Sell. Existence of dichotomies and invariant splittings for linear differential systems. II. J. Differential Equations, 22(2):478-496, 1976.

[37] W. Shen. On the measurable dynamics of real rational functions. Ergodic Theory Dynam. Systems, 23(3):957-983, 2003.

[38] W. Shen. Decay of geometry for unimodal maps: an elementary proof. Ann. of Math. (2), 163(2):383-404, 2006.

[39] D. Smania. Complex bounds for multimodal maps: bounded combinatorics. Nonlinearity, 14(5):1311-1330, 2001.

[40] D. Smania. Phase space universality for multimodal maps. Bull. Braz. Math. Soc. (N.S.), 36(2):225-274, 2005.

[41] D. Smania. On the hyperbolicity of the period-doubling fixed point. Trans. Amer. Math. Soc., 358(4):1827-1846 (electronic), 2006.

[42] D. Smania. Puzzle geometry and rigidity: the Fibonacci cycle is hyperbolic. J. Amer. Math. Soc., 20(3):629-673 (electronic), 2007.

[43] D. Smania. Shy shadows of infinite-dimensional partially hyperbolic invariant sets. Ergodic Theory Dynam. Systems, 39(5):1361-1400, 2019.

[44] D. Sullivan. Bounds, quadratic differentials, and renormalization conjectures. In American Mathematical Society centennial publications, Vol. II (Providence, RI, 1988), pages 417-466. Amer. Math. Soc., Providence, RI, 1992.

[45] C. Tresser and P. Coullet. Itérations d'endomorphismes et groupe de renormalisation. $C$. $R$. Acad. Sci. Paris Sér. A-B, 287(7):A577-A580, 1978.

Departamento de Matemática, Instituto de Ciências Matemáticas e de ComputaÇão, Universidade de São Paulo-Campus de São Carlos (ICMC/USP/São Carlos), Caixa Postal 668, CEP 13560-970, SÃo CARLOS-SP, BrazIL

E-mail address: smania@icmc.usp.br

URL: http://conteudo.icmc.usp.br/pessoas/smania/ 